ETHZ-IPP-2010-07

July 2010

\title{
Improving Higgs Sensitivity at CDF by Introducing New Muon Triggers
}

\author{
MASTER THESIS
}

presented by

\section{Marco - Andrea Buchmann}

Prof. Dr. G. Dissertori, ETH Zürich, Supervisor

Prof. Dr. R. Wallny, University of California, Los Angeles, Supervisor at Fermilab Prof. Dr. C. Group, University of Virginia, Mentor at Fermilab

Institute for Particle Physics

ETH Zürich

Switzerland 


\section{Abstract}

A search for Standard Model Higgs boson production in the $H \rightarrow W W \rightarrow l \nu j j$ channel using $5.7 \mathrm{fb}^{-1}$ of CDF II data is presented. The search is performed using a matrix element technique in which event probability densities for the signal and background hypotheses are calculated and used to create a powerful disciminator (called the event probability discriminant, EPD). The EPD distributions for signal and background are fit to the data using a binned likelihood approach to search for the Higgs boson signal. To improve the limits on the $H$ production cross section, a new muon category, CMP, is added.

No evidence for a Higgs boson signal is observed, and 95\% confidence level upper limits on the $H$ cross section times the branching ratio of the Higgs boson to decay to two $W$ of $\sigma(p \bar{p} \rightarrow H) \times B R(H \rightarrow W W) / S M<7.7$ to 62.1 for Higgs boson masses of between $m_{H}=150 \mathrm{GeV} / c^{2}$ and $m_{H}=200 \mathrm{GeV} / c^{2}$ are found. The expected (median) limit estimated in pseudo-experiments is $\sigma(p \bar{p} \rightarrow H) \times B R(H \rightarrow W W) / S M<12.5$ to 41.0 at 95\% C.L.

The inclusion of the phi gap trigger lead to improvements in the sensitivity of up to $7.3 \%$; on average, the improvement in the sensitivity was $3.3 \%$.

This analysis was blessed by the CDF collaboration on July 31, 2010. 


\section{Contents}

1 Introduction 6

1.1 The Standard Model . . . . . . . . . . . . . . . . . . . . . . . . . . . . 6

1.1.1 The Particles in the Standard Model . . . . . . . . . . . . . . 6

1.1 .2 Hadronization . . . . . . . . . . . . . . . . . . 7

1.2 The Higgs Particle . . . . . . . . . . . . . . . . . . . . . . . 9

1.2 .1 The Higgs Mechanism . . . . . . . . . . . . . . . . . . . . . . . . . . 9

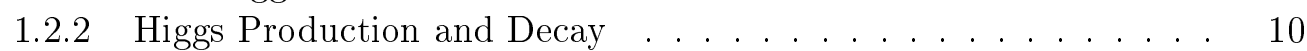

$1.2 .3 \quad$ Experimental Findings . . . . . . . . . . . . . . . . . . . . . . . . . . . . . . . . . . . .

1.3 Cross Section Calculations $\ldots \ldots \ldots \ldots$. . . . . . . . . . . . 11

\begin{tabular}{lll}
\hline 2 & Experimental Apparatus & 14
\end{tabular}

2.1 The Tevatron[2][3] . . . . . . . . . . . . . . . . . . . . . . . . 14

2.2 The CDF II Detector [6] . . . . . . . . . . . . . . . . . . . . . . . 15

2.2 .1 The Coordinate System . . . . . . . . . . . . . . . 16

2.2 .2 The Beam Pipe[6] . . . . . . . . . . . . . . . . . . . . . . . . . . . . . . . . . .

2.2 .3 The Silicon Detector[6][7] . . . . . . . . . . . . . . . . . 16

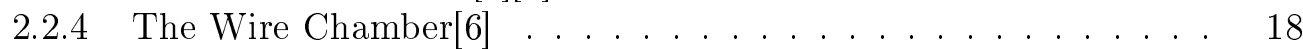

2.2 .5 The Time of Flight System[15] . . . . . . . . . . . . . . . . . . . 19

2.2 .6 The Solenoid Magnet[6] . . . . . . . . . . . . . . . . . . . . . . . . . 19

$2.2 .7 \quad$ The Calorimeters 6 [6] $[8 \ldots \ldots \ldots$. . . . . . . . . . . . . . . . . 19

2.2 .8 The Muon Detectors [6][9] . . . . . . . . . . . . . . . . . . . 20

2.3 Data Sample . . . . . . . . . . . . . . . . 22

\begin{tabular}{lll}
3 & New Categories & 24 \\
\hline
\end{tabular}

$3.1 \quad$ Coverage of $\eta-\phi$ Space . . . . . . . . . . . . . . . . . . . . . 24

3.2 CMP Identification Cuts $\ldots \ldots \ldots \ldots \ldots \ldots$

3.3 Definitions of the New Categories . . . . . . . . . . . . . . . . 26

\begin{tabular}{llr}
\hline & Event Selection & 29
\end{tabular}

4.1 Backgrounds and Vetoes . . . . . . . . . . . . . . . . . . . . . 29

$4.1 .1 \quad W+$ Jets $\ldots \ldots \ldots \ldots \ldots$

4.1 .2 Top Production . . . . . . . . . . . . . . . . . . . . . . . 30

$4.1 .3 \quad$ QCD Multijet . . . . . . . . . . . . . . . . . . . . . . . . 31

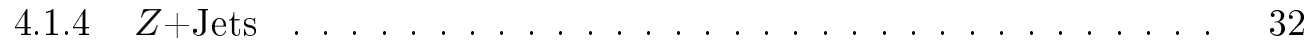

4.1 .5 Diboson . . . . . . . . . . . . . . . . . . . . 32

4.1 .6 Cosmic Ray Veto . . . . . . . . . . . . . . . . . . . 32

4.1 .7 Additional Cuts . . . . . . . . . . . . . . . . . . . . . . 33

4.2 Trigger Paths $\ldots \ldots \ldots \ldots \ldots$. . . . . . . . . . . . . . . . . . . . . 34

4.3 Offline Event Selection . . . . . . . . . . . . . . . . . . . . . . . . . . 37

5 Monte Carlo Modeling 38

5.1 Monte Carlo Samples . . . . . . . . . . . . . . . . . . . . . . . . 38

5.2 Background Estimate $\ldots \ldots \ldots \ldots \ldots$. . . . . . . . . . . . 38

$5.2 .1 \quad$ MC Based Background Estimate . . . . . . . . . . . . . . . 39 


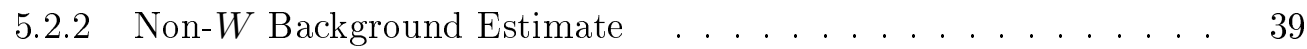

5.2 .3 Signal Estimate . . . . . . . . . . . . . . . . . . . . . . 41

5.3 Event Yields . . . . . . . . . . . . . . . . . . . . . . . 43

5.4 Modeling Validation . . . . . . . . . . . . . . . . . . . . . . 43

6 Matrix Element Method 57

$6.1 \quad$ Event Probabilities . . . . . . . . . . . . . . . . . . . . . . . . 57

6.2 Transfer Function . . . . . . . . . . . . . . . . . . . . . 59

6.3 Event Probability Discriminant . . . . . . . . . . . . . . . . . . . . . . 59

6.4 Signal and Background Processes . . . . . . . . . . . . . . . . . 60

6.5 Effectiveness of the Event Probability Discriminant . . . . . . . . . . . 63

6.6 The Likelihood Fit . . . . . . . . . . . . . . . . . . . . . . . . . 64

$6.6 .1 \quad$ Fitting Procedure . . . . . . . . . . . . . . . . . 64

$\begin{array}{lll}7 & \text { Systematic Uncertainties } & 67\end{array}$

7.1 Background Normalization . . . . . . . . . . . . . . . . . . . . . 67

7.2 Signal Normalization . . . . . . . . . . . . . . . . . . . . . 68

7.3 Jet Energy Scale $\ldots \ldots \ldots \ldots$. . . . . . . . . . . . . . . . 68

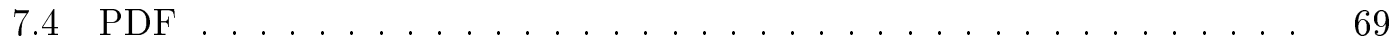

7.5 Initial State Radiation / Final State Radiation . . . . . . . . . . . . . . 69

7.6 Factorization and Renormalization Scale . . . . . . . . . . . . . . . . . 69

7.7 Event Selection Efficiency and Luminosity . . . . . . . . . . . . . . . . . 69

$\begin{array}{lll}8 & \text { Results } & \mathbf{7 1}\end{array}$

$8.1 \quad$ Effect of Systematic Uncertainties . . . . . . . . . . . . . . . . . . . . 74

8.1 .1 Upper Limits without Systematic Uncertainties . . . . . . . . . . 74

8.1.2 Effect of Uncertainty on the $W+$ jets Normalization . . . . . . . . 74

$8.1 .3 \quad$ Effect of Uncertainty on Jet Energy Scale . . . . . . . . . . . . . 74

8.1.4 Effect of Uncertainty on Factorization/Renormalization Scale . . 75

8.1.5 Summary of Effects of Systematic Uncertainties . . . . . . . . . . . . 75

8.2 Effect of the Addition of the Phi Gap Trigger . . . . . . . . . . . . . . 76

\begin{tabular}{lll}
\hline & Conclusions and Outlook & 77
\end{tabular}

A Higgs Field and Gauge Boson \& Fermion Masses 80

A.1 Gauge Boson Masses in $S U(2) \otimes U(1)$. . . . . . . . . . . . . . . . . . . 81

A.2 Fermion Masses . . . . . . . . . . . . . . . . . . . . . . . . . . . 82 
Dedicated to my grand parents whose iron will and determination in the face of tremendous challenges has always inspired me

Meinen Grosseltern gewidmet, deren Entschlossenheit und Stärke im Angesicht grösster Herausforderungen mich stets inspiriert hat 


\section{Introduction}

The Standard Model of particle physics is a remarkably successful description of the interaction of fundamental particles and has been extensively tested at the Tevatron. One of the most fundamental problems in particle physics is understanding the mechanism which breaks the electroweak symmetry and generates the mass of all known elementary particles. The prime candidate for this is the Higgs boson.

Prospective Higgs events do not have a very clear signature and they are burried under background processes that are dominating by orders of magnitude. For some of the most promising Higgs production and decay channels, such as $W H \rightarrow l \nu b \bar{b}, Z H \rightarrow l l b \bar{b}$ and $H \rightarrow W W$, the lepton in the final state is used to distinguish them from the overwhelming QCD background. Searches for the Higgs at the Tevatron have been mainly statistically limited[1], and searches for small effects in general require very sophisticated methods which take advantage of every available piece of information which is why it is very important to maximize the acceptance of the analysis.

In this Master thesis, an analysis of the $H \rightarrow W W \rightarrow l \nu j j$ channel is presented, and a new muon trigger is added to improve Higgs sensitivity. The major challenge is distinguishing Higgs events from the vast backgrounds, particularly $W+$ jets. The analysis is closely related to the measurement of the $W W+W Z$ cross section in a semileptonic decay mode (CDF Note 10124[17]). Matrix Element calculations are used to separate the signal events from the backgrounds.

In the remainder of this chapter, the theoretical concepts relevant to this analysis are laid out, followed by the experimental context and apparatus in Chapter 2. The next chapter describes the new CMP lepton category and other changes to the categories made necessary by the inclusion of the new muon trigger. The following chapter details the event selection, followed in turn by details on the Monte Carlo Modeling in Chapter 4 . In the ensuing chapter, the matrix element method is explained, which in turn is followed by an overview of systematic uncertainties in the analysis. In the last two chapters, the results and the conclusion are presented.

\subsection{The Standard Model}

The Standard Model is a quantum field theory of the strong $(S U(3))$ and electroweak $(S U(2) \otimes U(1))$ interactions with the gauge group $S U(3) \otimes S U(2) \otimes U(1)$ and requires the Lagrangian to be invariant under the corresponding local transformations.

\subsubsection{The Particles in the Standard Model}

The particles of the Standard Model can be split up into two distinct groups based on their spin: fermions and bosons. Fermions have half-integer spin whereas bosons have integer spin.

The fermions can be split up into three groups of particles, each containing a quark 
with charge $+\frac{2}{3}$, a quark with charge $-\frac{1}{3}$, a lepton with charge -1 and an uncharged neutrino (the particles of the three generations are listed in Table 1).

The gauge bosons act as mediators of the interactions: The photon $(\gamma)$ couples to charged particles, the corresponding interaction is the electromagnetic interaction which is described by the $U(1)$ symmetry. The $S U(2)$ symmetry describes the weak interaction, mediated by the massive $W$ and $Z$ gauge bosons. One peculiarity of the weak interaction is that it acts exclusively on left-handed particles and right-handed anti-particles ("violates parity maximally"); furthermore, it is the only interaction capable of changing the flavor. Finally, the gluons couple to color charge in the so-called strong interaction which is described by the $S U(3)$ symmetry. Due to the fact that $S U(3)$ is non-Abelian, self-interactions are possible and the gluon itself has a color charge.

The last of the four fundamental interactions is gravity; it has not been successfully introduced into the Standard Model yet, though its strength is almost thirty orders of magnitude less than that of the weak force, so it can be safely neglected.

\subsubsection{Hadronization}

QCD perturbation theory is valid only at short distances; at larger distances, the strong coupling constant $\alpha_{S}$, which is the expansion parameter, becomes large and perturbation theory consequently breaks down. Partons are "confined", i.e. they cannot be isolated singularly or directly observed but transform into colorless hadrons in a process called hadronization. There are several models aimed at explaining the process which is still not fully derived form first principles (such as string fragmentation (SF), independent fragmentation (IF) and cluster fragmentation (CF)).

When partons reach a certain distance from each other, it becomes energetically favorable to produce a new quark-antiquark pair out of the vacuum instead of separating the two original quarks further. In the detector, one thus observes collections of particles moving in the same direction, so-called jets, rather than isolated partons. The constituents of jets are hadrons that consist of three quarks or anti-quarks ("baryons") or of a quark-antiquark pair ("mesons"). The process of hadronization with the different stages, from parton to detector signal, is illustrated in Fig. 1.

\begin{tabular}{|c|c|c|c|c|c|c|c|}
\hline & Charge & \multicolumn{2}{|c|}{$1^{\text {st }}$ generation } & \multicolumn{2}{c|}{$2^{\text {nd }}$ generation } & \multicolumn{2}{|c|}{$3^{\text {rd }}$ generation } \\
\hline \multirow{2}{*}{ Quarks } & $+\frac{2}{3}$ & Up & $\mathrm{u}$ & Charm & $\mathrm{c}$ & Top & $\mathrm{t}$ \\
& $-\frac{1}{3}$ & Down & $\mathrm{d}$ & Strange & $\mathrm{s}$ & Bottom & $\mathrm{b}$ \\
\hline \multirow{2}{*}{ Leptons } & -1 & Electron & $\mathrm{e}$ & Muon & $\mu$ & Tau & $\tau$ \\
& 0 & Electron neutrino & $\nu_{e}$ & Muon neutrino & $\nu_{\mu}$ & Tau neutrino & $\nu_{\tau}$ \\
\hline
\end{tabular}

Table 1: The three generations of fermions in the Standard Model 


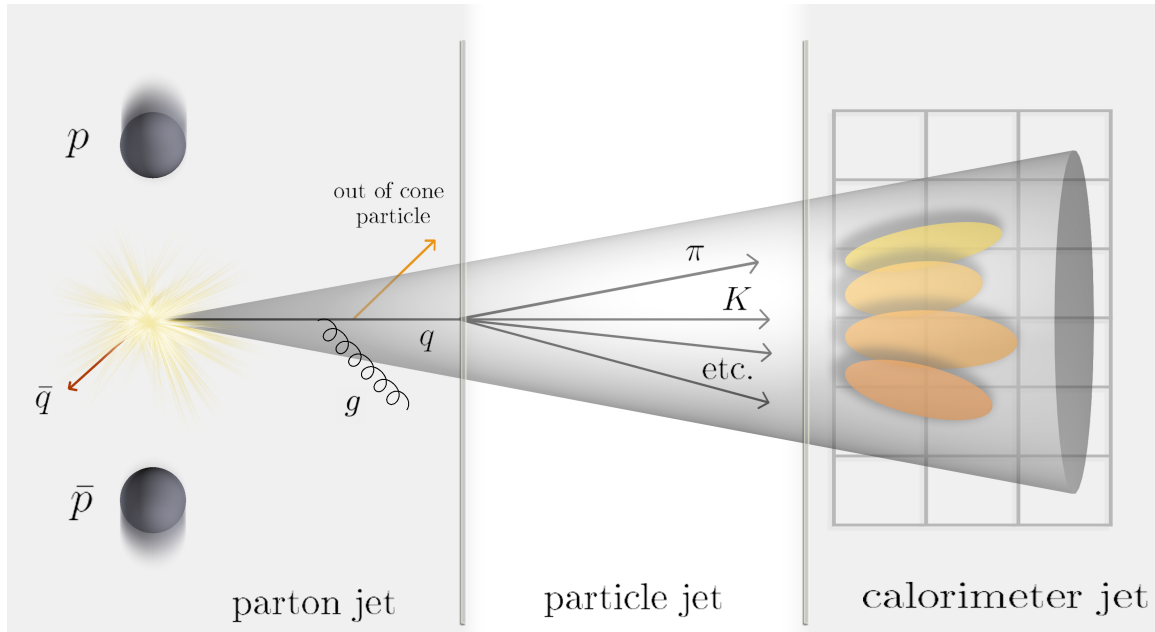

Figure 1: Hadronization: in the interaction, partons are formed which hadronize to give particle jets which are registered in the detectors and reconstructed. 


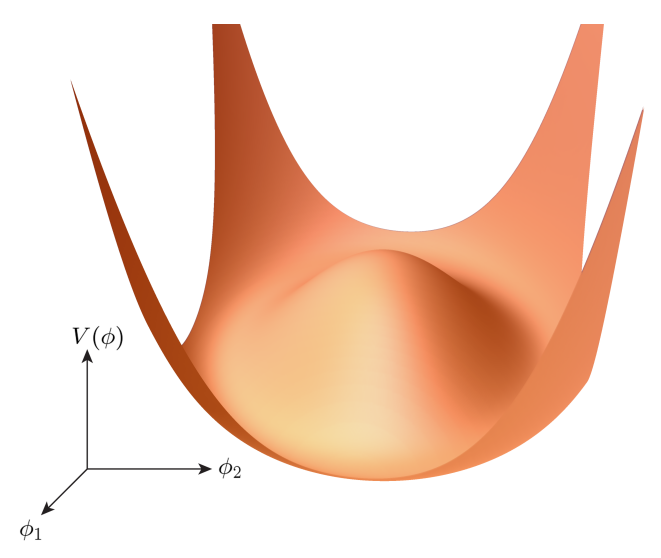

Figure 2: Illustration of the Mexican hat potential $V(\phi)=\mu^{2} \phi^{\dagger} \phi+\lambda\left(\phi^{\dagger} \phi\right)^{2}$ with $\lambda>0$, $\mu^{2}<0$. It features a circle of minima at $|\phi|^{2}=-\frac{\mu^{2}}{2 \lambda}$.

\subsection{The Higgs Particle}

The Higgs mechanism is the prime candidate to explain how the electroweak symmetry is borken and how the mass of all known elementary particles is generated. In the following, a short sketch of the Higgs mechanism is presented to illustrate that the Higgs coupling is proportionate to the mass of the corresponding particle (more details can be found in Appendix A). The overview is followed by a description of the higgs production and decay channels, which in turn is followed by a summary of previous direct and indirect searches, and the excluded mass ranges.

\subsubsection{The Higgs Mechanism}

The key idea behind the Higgs mechanism is the spontaneous breaking of the electroweak symmetry 1 . The theory remains renormalizable even in the presence of spontaneous symmetry breakdown (as shown by 't Hooft in 1971). The objective is to avoid the massless vector particles that arise with the imposition of local symmetry and obtain instead massive vector particles in accordance with experimental findings. In the Abelian case, one starts with a complex scalar field $\phi$ and the potential

$$
V(\phi)=\mu^{2} \phi^{\dagger} \phi+\lambda\left(\phi^{\dagger} \phi\right)^{2}
$$

which for $\lambda>0, \mu^{2}<0$ becomes the so-called Mexican hat potential (an illustration of the potential is given in Fig. 22 with a circle of minima at $|\phi|^{2}=-\frac{\mu^{2}}{2 \lambda}=: \frac{1}{2} v^{2}$. When the covariant derivative in $S U(2) \otimes U(1)$

$$
D_{\mu}=\partial_{\mu}-i g \frac{1}{2} \vec{\tau} \vec{W}_{\mu}-i g^{\prime} \frac{1}{2} Y B_{\mu}
$$

is used, a look at the particle spectrum of $\left[D_{\mu} \phi\right]^{\dagger}\left[D^{\mu} \phi\right]$ for $\phi=\frac{1}{\sqrt{2}}\left(\begin{array}{l}0 \\ v\end{array}\right)$ reveals that $m_{W}=\frac{1}{2} v g$ and $m_{Z}=\frac{1}{2} v \sqrt{g^{2}+g^{\prime 2}}$. For fermions, the interaction between matter and

\footnotetext{
${ }^{1}$ According to Goldstone's theorem, the spontaneous breaking of a continuous symmetry leads to scalar massless (or in the case of an inexact symmetry, light) particles appearing in the spectrum of possible excitations
} 


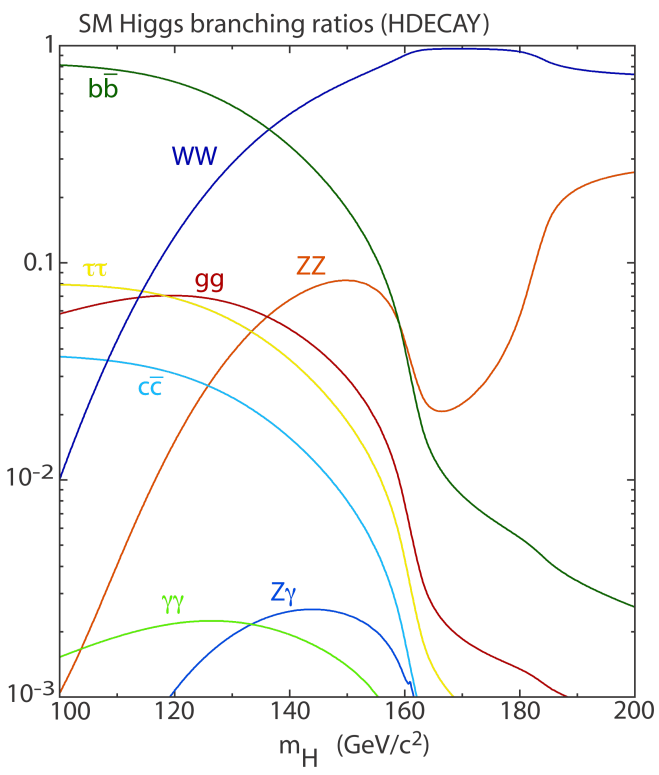

(a) Standard Model Higgs branching ratios for a Higgs mass between $100 \leq m_{H} \leq 200$ $\mathrm{GeV}$ (computed using HDECAY)

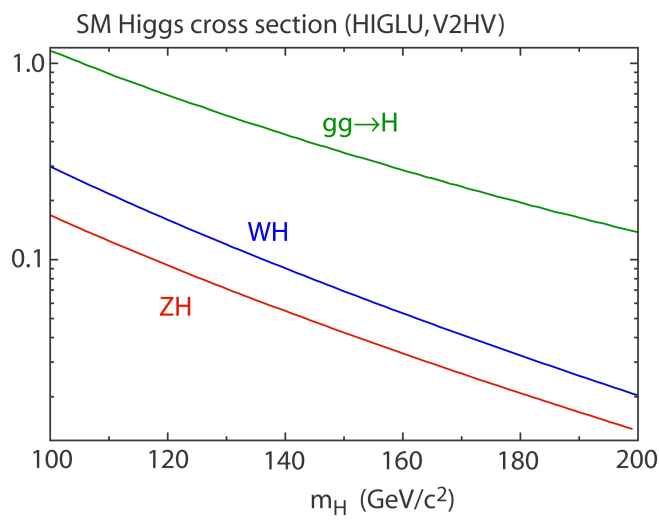

(b) Standard Model Higgs cross section for a Higgs mass between $100 \leq m_{H} \leq 200 \mathrm{GeV}$ (computed using HIGLU, V2HV)

Figure 3: Higgs branching ratios and cross section [10][11]

the Higgs field yields couplings that are proportionate to the mass of the fermion, e.g. for electrons $\left(e_{L}\right.$ with $\left.T_{3}=+\frac{1}{2}\right)$ the gauge invariant term

$\left.\mathcal{L}_{m}=-G_{e}\left[\begin{array}{ll}\left(\bar{\nu}_{e}\right. & \bar{e}\end{array}\right)_{L}\left(\begin{array}{c}\phi^{+} \\ \phi^{0}\end{array}\right) e_{R}+\bar{e}_{R}\left(\bar{\phi}^{+} \quad \bar{\phi}^{0}\right)\left(\begin{array}{c}\nu_{e} \\ e\end{array}\right)_{L}\right] \quad, \quad$ with $\left(\begin{array}{c}\phi^{+} \\ \phi^{0}\end{array}\right)=\frac{1}{\sqrt{2}}\left(\begin{array}{c}0 \\ v+h(x)\end{array}\right)$

is included, where $G_{e}$ is the electronic Yukawa coupling. Thus,

$$
\mathcal{L}_{m}=-\frac{G_{e}}{\sqrt{2}} v\left(\bar{e}_{L} e_{R}+\bar{e}_{R} e_{L}\right)-\frac{G_{e}}{\sqrt{2}} h\left(\bar{e}_{L} e_{R}+\bar{e}_{R} e_{L}\right)=-m_{e} \bar{e} e-\frac{m_{e}}{v} \bar{e} e h
$$

where the electron mass

$$
m_{e}=\frac{G_{e} v}{\sqrt{2}}
$$

was introduced. One can thus directly see that the coupling eeh is proportionate to the mass of the electron (and more generally, proportionate to the mass of the fermion, see Appendix A. The fermion masses themselves however are not predicted and remain free parameters of the Standard Model.

\subsubsection{Higgs Production and Decay}

There are several ways in which a Higgs boson can be produced, namely gluon fusion $g g \rightarrow H, t \bar{t}$ fusion $t \bar{t} \rightarrow H$, Higgs strahlung $q \bar{q} \rightarrow W H(Z H)$ and vector boson fusion. These modes have cross sections that are dependent on the mass of the Higgs boson as illustrated in Fig. 3b. The dominant contribution comes from gluon fusion over the considered mass range of $100 \leq m_{H} \leq 200 \mathrm{GeV}$; Feynman diagrams at leading order for 
the different production processes are shown in Fig. 4 .

There are not only several modes for the Higgs particle's production but also for its decay; the Higgs particle's branching ratios for masses between $100 \leq m_{H} \leq 200 \mathrm{GeV}$ are illustrated in Fig. 3a. One can see that up to $135 \mathrm{GeV}$, the $b \bar{b}$ channel is dominant ("light Higgs") after which the $W W$ channel becomes dominant ("heavy Higgs") on which the analysis presented here is focussed.

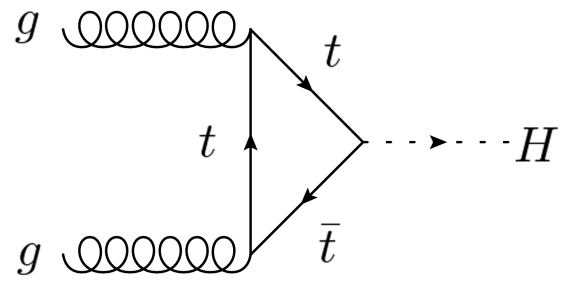

(a) Gluon fusion

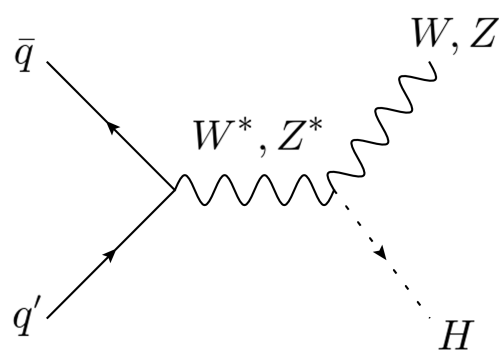

(c) Higgs strahlung

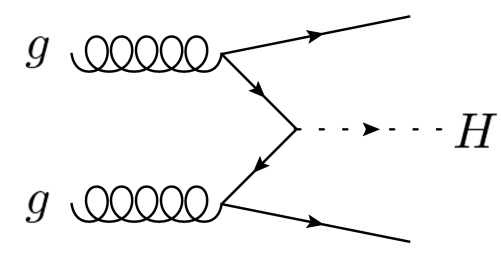

(b) $t \bar{t}$ fusion

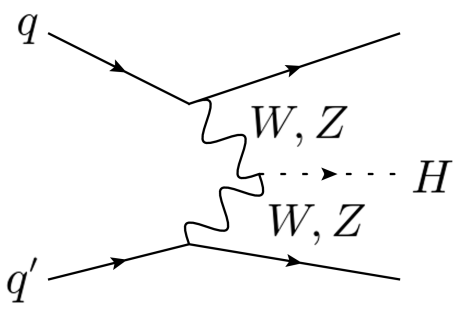

(d) Vector boson fusion

Figure 4: Leading order Feynman diagrams for Higgs production

\subsubsection{Experimental Findings}

The Higgs model has been extensively studied over the past decades. Prior to 1989, searches for the Higgs boson were mostly sensitive to candidates with masses below a few $\mathrm{GeV}$. During LEP2, the energy was increased and the combined data from all experiments was sensitive to neutral Higgs boson candidates with masses up to $115 \mathrm{GeV}$ and to charged Higgs boson candidates with masses up to about $90 \mathrm{GeV}$. The search was continued at the Tevatron and the reach was extended. In summary, the previous direct searches carried out at LEP and at the Tevatron have set 95\% C.L. limits, constraining the Higgs mass to between 114.4 and $158 \mathrm{GeV}$, or above $175 \mathrm{GeV}[12]$ [23] (see Fig. 5). Furthermore, precision electroweak measurements have hinted at an upper bound of less than $186 \mathrm{GeV}$ at $95 \%$ C.L. [14] for the Higgs mass.

\subsection{Cross Section Calculations}

The cross section of an interaction is a measure of the probability of said interaction taking place. It is originally based on the geometric interpretation of the classical picture of 


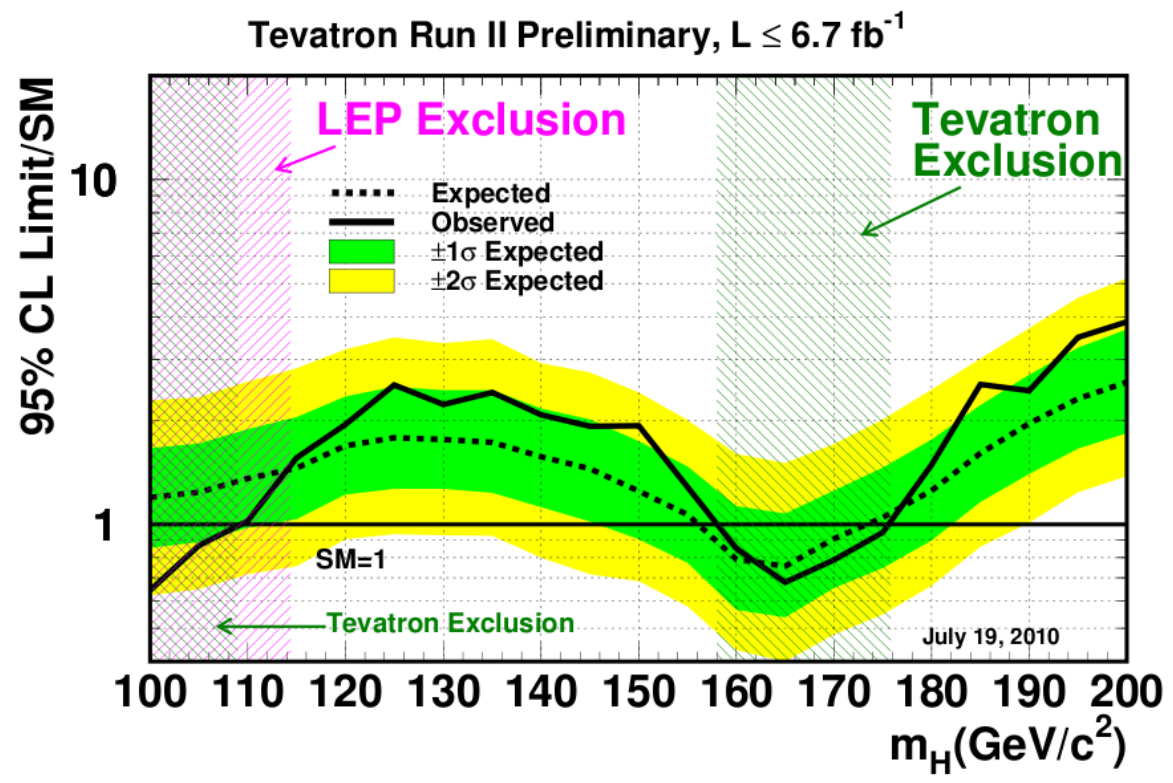

Figure 5: Illustration of the observed and expected $95 \%$ confidence level upper limits on the ratios to the Standard Model cross section as functions of the Higgs boson mass for the combined CDF and DØ analyses. The bands indicate the $68 \%$ and $95 \%$ probability regions where the limits can fluctuate in the absence of a signal. [23]

point-like projectiles hitting a solid target and still carries the unit of an area $(1 \mathrm{barn}=$ $10^{-28} \mathrm{~m}^{2}$ ). The cross section cannot be evaluated exactly but a perturbative expansion in powers of a coupling constant is used instead ${ }^{2}$ Each of the terms in the expansion can be graphically represented by Feynman diagrams; by evaluating these diagrams and summing, one obtains the amplitude of the process.

The expansion in terms of the coupling constant is also an expansion in the number of loops; the first term is the leading order (LO) contribution, the corresponding Feynman diagrams are referred to as "tree diagrams" and have no loops. This first term gives a good first approximation. Diagrams induced by the second term in the expansion contain one loop, and their evaluation leads to corrections (NLO corrections) to the approximation from the LO calculation. The next step is the evaluation of NNLO diagrams, i.e. calculating two-loop corrections.

The presence of loops leads to integrals over momentum space which can entail divergences (often referred to as infrared divergences). These can be dealt with by renormalization. One then finds a finite result that approximates the experiment well. However, the introduction of a renormalization scale causes couplings to depend on the energy scales; this introduces a problem and some uncertainty in the calculation of cross sec-

\footnotetext{
${ }^{2}$ The cross section is thus only accessible by the perturbative expansion if the expansion parameter $\alpha$ is smaller than one which is not the case for instance for hadronization
} 
tions or the generation of simulated events. Another type of divergence arises at the other end of the momentum scale, for small momenta ("infrared divergences"). When the expansion parameter approaches 1 or is even larger than 1 , the perturbative description breaks down. These divergences are accounted for by introducing a factorization scale truncating the integral before it reaches the non-perturbative region.

After summing over all connected and topologically distinct Feynman diagrams one obtains a value which is only valid for a given momentum and energy; one thus computes the differential cross section and after integrating for all final state momenta one obtains the total cross section for the interaction. 


\section{Experimental Apparatus}

\subsection{The Tevatron[2][3]}

The Tevatron is the world's second highest energy accelerator with a circumference of $6.28 \mathrm{~km}$, located at Fermi National Accelerator Laboratory (FNAL), near Chicago, IL. It collides protons and antiprotons at a center of mass energy of $\sqrt{s}=1.96 \mathrm{TeV}$. There have been numerous upgrades since the completion of its construction in 1983. After its commissioning, a run with only CDF was carried out in 1988-1989. The first joint run ("run 1", from 1992-1996) with a second detector, DØ, operated at an energy of $1.8 \mathrm{TeV}$ and delivered an integrated luminosity of more than $160 \mathrm{pb}^{-1}$. [4]. It had $6 \times 6$ proton and antiproton bunches with $3.5 \mu \mathrm{s}$ between crossings and luminosities on the order of $10^{31} \mathrm{~cm}^{-2} \mathrm{~s}^{-1}$. It collected approximately $120 \mathrm{pb}^{-1}$ per experiment. Following Run I, several major upgrades were carried out until 2001 when Run II commenced at an energy level of $1.96 \mathrm{TeV}$. Today, the luminosity achieved is on the order of $5.2 \cdot 10^{32} \mathrm{pb}^{-1} \mathrm{~s}^{-1}$. Furthermore, in Run II the bunches have been increased to $36 \times 36$ with 396 ns between crossings and there are approximately $2.7 \cdot 10^{11}$ protons (and $1.0 \cdot 10^{11}$ antiprotons) per bunch. 5

There have been numerous discoveries at the Tevatron, most notably the discovery of the top quark (in 1995), the first measurement of the $B_{s}$ oscillations in 2006, and the discovery of the single top in 2009.

The particles are kept on track by superconducting magnets generating a magnetic field of $4.2 \mathrm{~T}$; in fact, the Tevatron was the world's first superconducting synchrotron. [3] Dipoles are used to bend the particle beams, quadrupoles to focus them and sextupoles are employed for chromatic corrections; the coils are made of Nb-Ti alloy wire.

The accerelation consists of several stages; in a first stage, $\mathrm{H}^{-}$ions are accelerated up to $750 \mathrm{keV}$ with a Cockcroft-Walton generator which is followed by a linear accelerator (where the energy is increased to $400 \mathrm{MeV}$ ). After the linear accelerator, the $\mathrm{H}^{-}$ ions are stripped of their electrons (converting the ions from $\mathrm{H}^{-}$to $\mathrm{H}^{+}$) by passing them through a thin Carbon layer. These protons are then accelerated up to $8 \mathrm{GeV}$ in a circular accelerator called the "Booster ring". In the next step, the protons enter the Main

Injector which accelerates them further but also delivers some of them for anti-proton production and accelerates the anti-protons coming from the anti-proton source. The final stage is the Tevatron, where protons and anti-protons travel in opposite directions and meet in the two interaction points, where detectors are located: CDF and DØ.

CDF: The Collider Detector at Fermilab (CDF) was the first detector and predates DØ. It has received two upgrades since its first data recording. The detector's layout is explained below in section 2.2. The main objectives as laid out in the Technical Design Report [6] are the characterization of the properties of the top quark, a global precision electroweak program, direct search for new phenomena, tests of perturbative QCD at NLO and large $Q^{2}$ and constraint of the CKM matrix with high statistics B decays. 


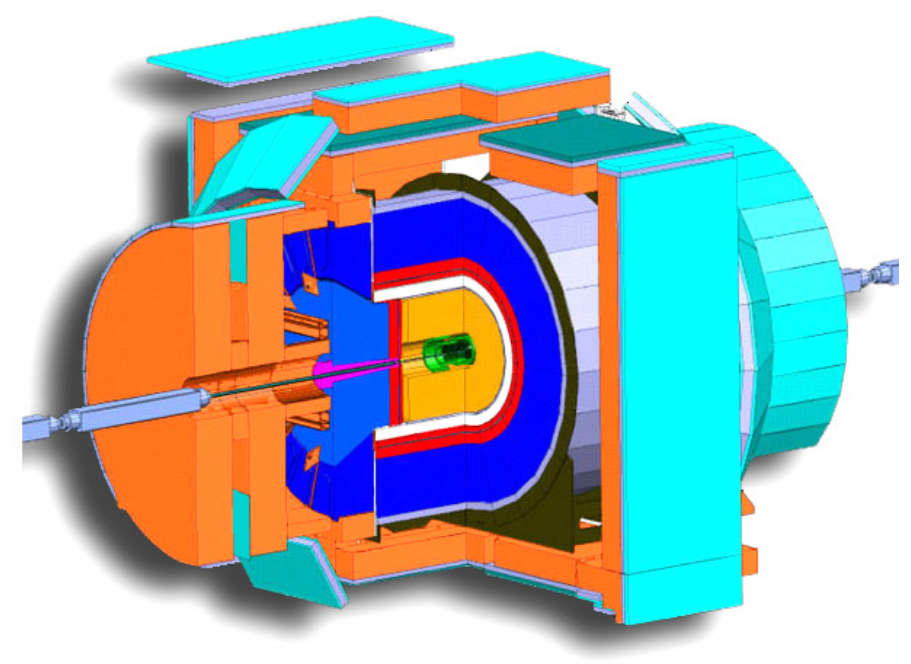

Figure 6: The CDF II Detector with all layers. The detector components are color coded as described in section 2.2 .

DØ: The DØ experiment is located at the second intersection of the beams. The research at $\mathrm{D} \varnothing$ has similar aims as CDF; it includes the search for new phenomena (including the Higgs boson), top physics, B physics, electroweak physics and several aspects of QCD.

In the following subsection, the CDF detector in Run II (also commonly referred to as CDF II detector) is presented, as the subsequent parts use data collected by the CDF II detector.

\subsection{The CDF II Detector [6]}

The CDF II detector is a general purpose solenoidal detector consisting of 7 layers. The layers illustrated in Fig. 6 are the following (color code in parentheses):

1. beam pipe (black and gray)

2. Silicon Detector (green)

3. Central Outer Tracker (light orange)

4. Superconducting Solenoid (white)

5. Electromagnetic Calorimeter (red)

6. Hadronic Calorimeter (blue)

7. Muon Detectors (turquoise)

In the following, these layers are explained in more detail. 

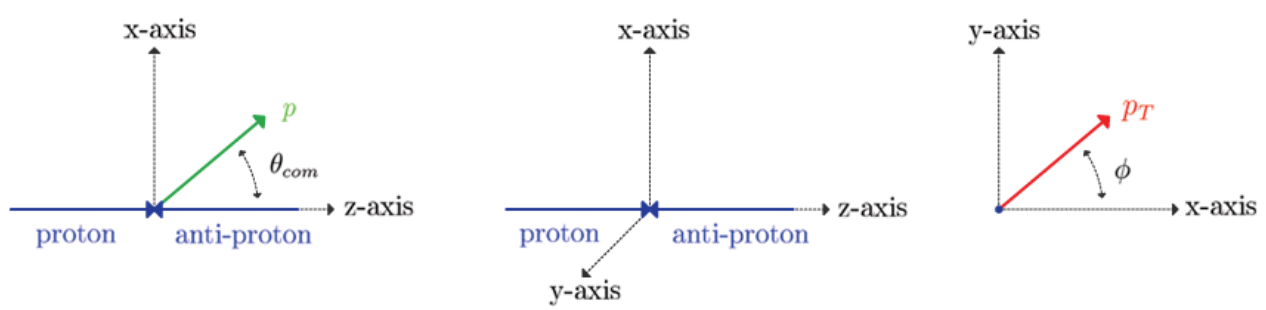

Figure 7: Illustration of the Tevatron coordinate system

\subsubsection{The Coordinate System}

In a Cartesian coordinate system, the $z$ axis comes to lie on the proton beam axis and the coordinate $z$ along the axis is measured from the nominal interaction point (as illustrated in Fig. 7). The $x-y$ plane is perpendicular to the beam axis; the corresponding component in vectors is referred to as the transverse component, as for instance with transverse momentum $p_{T}$. In most cases, a cylindrical coordinate system is preferred, with the distance from the nominal interaction point denoted by $r$ and the azimuthal angle $\phi$ being measured from the Tevatron plane. Furthermore, one often introduces the polar angle $\theta$ which is measured from the proton direction upwards, and the pseudo-rapidity $\eta=-\ln \left(\tan \left(\frac{\theta}{2}\right)\right)$.

\subsubsection{The Beam Pipe[6]}

The innermost layer of the detector is the beam pipe which is $343 \mathrm{~cm}$ long. It is made of three parts of which the central part is located inside the CDF II detector. The beam pipe has an outer radius of $1.2 \mathrm{~cm}$ and has $0.050 \mathrm{~cm}$ thick walls; it is made of Beryllium which has a low atomic number thereby avoiding scattering and thus significantly reducing background rates.

Both sides of the beam pipe have a low mass stainless steel bellows pipe which connects the beryllium pipe with the low beta quadrupole magnet. A low mass flange links the beryllium pipe with the bellows pipe.

\subsubsection{The Silicon Detector[6][7]}

A silicon strip detector is used for tracking purposes. It is designed for precision tracking as well as for reconstructing primary and displaced vertices. The silicon detector has an approximate $7 \mathrm{~m}^{2}$ of Silicon sensors which pertain to three groups of layers in the radial direction: Layer 00 (L00), SVX-II and the Intermediate Silicon Layers (ISL) (in order

of increasing radial distance from the nominal interaction point), as illustrated in Fig. $8 \mathrm{a}$ and Fig. 8b. 


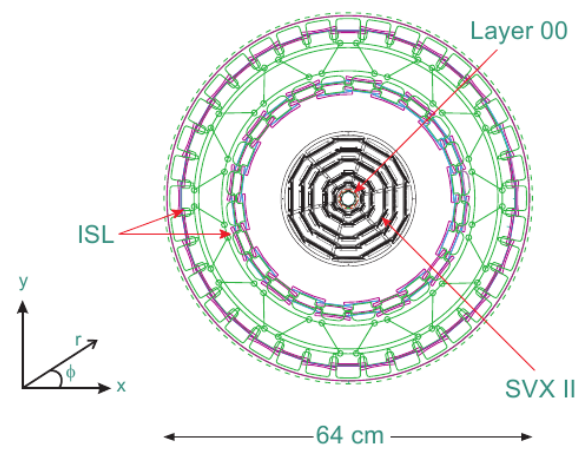

(a) $r \phi$ view of the CDF Si detector, with L00 in the center, followed by SVX-II and ISL

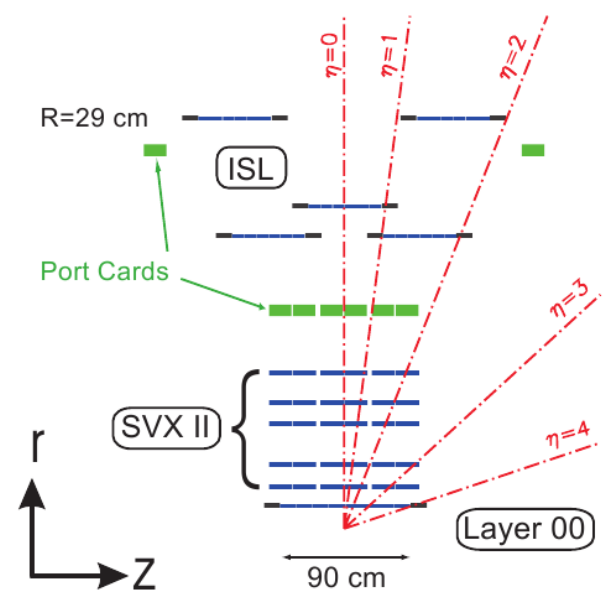

(b) $r-z$ view of the CDF Si detector. This figure shows the arrangement of the different layers for each sub-unit (note that the $z$ scale is compressed)

Figure 8: Illustration of the layout of the sub-units of the CDF Silicon detector

L00: The innermost layer, Layer 00 (also called L00) consists of single-sided silicon strips and is located at distances of only 1.35 and $1.62 \mathrm{~cm}$ from the interaction point (the beam pipe's outer radius is $1.2 \mathrm{~cm}$ ). Its proximity to the interaction point allows for spatial measurements even before scattering has occurred. On the other hand, the vicinity to the interaction point also entails elevated radiation levels, which made the use of SVX II material impossible as it would not have endured the radiation for long.

SVX II: The $95 \mathrm{~cm}$ long Run II Silicon Vertex Detector (SVX II) is the core element of the silicon detector. It provides track information to $|\eta|<2$, covers approximately $2.5 \sigma$ of the luminous region and consists of twelve wedges in $\phi$ with five layers each, containing double sided silicon microstrip detectors (between radii of 2.4 and $10.7 \mathrm{~cm}$ ). The double sided detectors provide $r-z$ readout for improved pattern recognition and $3-\mathrm{D}$ vertex reconstruction with an impact parameter resolution $\sigma_{\phi}<30 \mu \mathrm{m}$ and $\sigma_{z}<60 \mu \mathrm{m}$ for central high momentum tracks. The radiation levels in SVX II require radiation hard electronics and sensors, so the readout chips were fabricated using radiation hard CMOS processing. The radiation level at the inner layer was expected to be approximately 0.5 Mrad per $\mathrm{fb}^{-1}$, and the silicon sensors were expected to last up to a total dose of $1.0-1.5$ Mrad.

ISL: The Intermediate Silicon Layers (abbreviated as ISL) were added to extend the forward coverage of the silicon detector to a pseudorapidity of 2.0 and to permit connecting tracks from the COT and SVX II. A single ISL layer was placed at a radius of 22 $\mathrm{cm}$ in the central region and in the plug region $(1.0 \leq|\eta| \leq 2.0)$ and two layers of silicon were installed at radii of 20 and $28 \mathrm{~cm}$. The less intense radiation found at larger radii allowed for longer strips to reduce the channel count. Six silicon based measurements in 


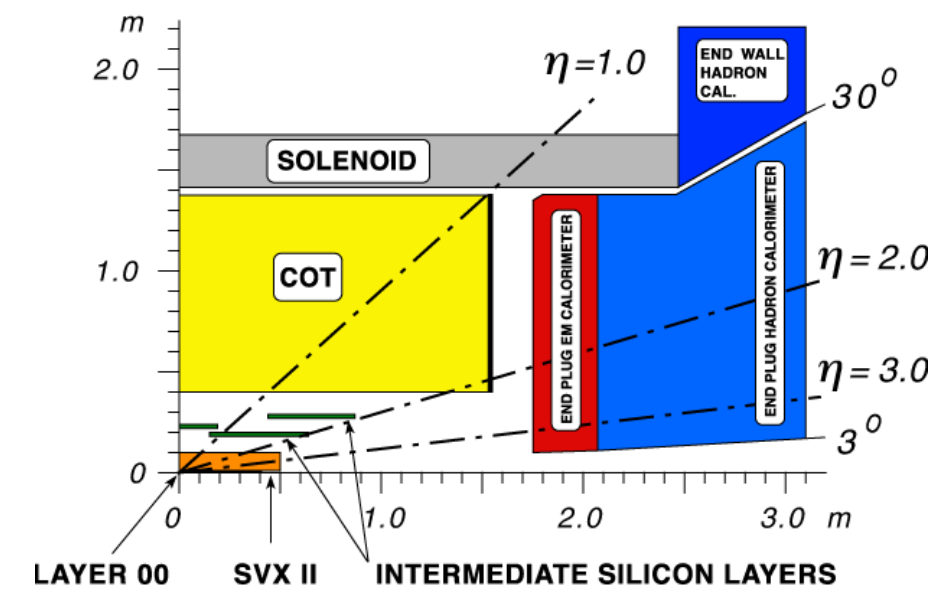

Figure 9: Radial location of the Central Outer Detector (COT)

the central region provide a stand-alone segment for optimized tracking in conjunction with the wire chamber (see section 2.2.4).

After some initial commissioning problems, the detector has been running reliably with a high tracking efficiency of $95 \%$.

\subsubsection{The Wire Chamber[6]}

The third layer after the beam pipe and the silicon detector is the central outer tracker (COT), an open cell drift chamber which covers radii between 44 and $132 \mathrm{~cm}$ and provides tracking at large radii in the central pseudorapidity region (i.e. $|\eta| \leq 1.0$ ). The central outer tracker is located in the radial region just outside the ISL but inside the solenoidal magnetic field.

The COT's active volume is $310 \mathrm{~cm}$ in the beam direction $z$ and between 44 and $132 \mathrm{~cm}$ in the radial direction, covering the entire azimuth $\phi$. Reconstructed tracks provide accurate data for the $r-\phi$ view (leading to precise data for $p_{T}$ ) whereas the $r-z$ view is significantly less accurate (thus also adversely affecting $\eta$ ).

The basic drift chamber has a line of twelve sense wires $(41 \mu \mathrm{m}$ gold-plated tungsten wires), the field wires were replaced by a cathode field panel (45 $\mathrm{nm}$ gold on both sides of a $6 \mu \mathrm{m}$ Mylar sheet). The ends of each cell are closed. In case one wire breaks within a cell, the damage is contained within the cell and does not affect other cells. There are four axial and four stereo superlayers which provide a total of 96 measurements. The COT's 32,240 sense wires are read out using pipelined TDCs.

The maximum drift time needed to be adapted from Run I, as the bunch spacing had decreased. Originally, a bunch spacing of $132 \mathrm{~ns}$ was envisioned (though only $396 \mathrm{~ns}$ was realized); the COT was thus designed to have a maximum drift time of 100 ns which was 
achieved by reducing the maximum drift distance. Furthermore, a different gas with a higher drift velocity was put in place. The gas currently used is a mixture containing 50:35:15 Ar-Et-CF 4 , which has a drift velocity of approx. $100 \mu \mathrm{m} / \mathrm{ns}$. The $\mathrm{CF}_{4}$ has been added as it has been found to reduce wire ageing in Ar-Et.

\subsubsection{The Time of Flight System[15]}

Wedged between the COT and the solenoid magnet is the time of flight system (TOF), which is a barrel located at a distance of $140 \mathrm{~cm}$ from the beam axis. The system consists of 218 scintillator bars which are $280 \mathrm{~cm}$ long and have a trapezoidal cross section of $4 \times 4 \mathrm{~cm}$ which each cover $1.7^{\circ}$ in $\phi$ and $|\eta|<1$. At the end of each bar, a photomultiplier tube (PMT) is attached. The PMT signal follows two paths, one for charge detection and one for the timing measurement. The TOF resolution is 120 ps which permits two standard deviation separation between $\mathrm{K}^{ \pm}$and $\pi^{ \pm}$for momenta $p<1.6 \mathrm{GeV} / \mathrm{c}$. The TOF information is also used in the L1 trigger to find magnetic monopoles.

\subsubsection{The Solenoid Magnet[6]}

The next layer is the magnetic solenoid. A cylindrical current induces an axial field of 1.5 T. The solenoid's useful volume has a radius of $1.4 \mathrm{~m}$ and measures $3.5 \mathrm{~m}$ in length. A high purity aluminium stabilized NbTi conductor is used which permits currents up to 5000 Amps. The magnet is cooled indirectly with liquid Helium and has been running with a field availability of $>99 \%$.

\subsubsection{The Calorimeters[6][8]}

The fifth and sixth layer in the radial direction, right after the solenoid, are the electromagnetic calorimeter and the hadronic calorimeter. These two calorimeters cover the region $|\eta| \leq 3.0$. Their location is indicated in Fig. 10a and Fig. 10b.

The electromagnetic calorimeter is a lead/scintillator sampling device which consists of wedges covering $15^{\circ}$. Each wedge is made up of 23 unit layers, yielding a total thickness of about $21 X_{0}$ (radiation length ${ }^{3}$ ) at normal incidence. The unit layer consists of 4.5 $\mathrm{mm}$ lead and $4 \mathrm{~mm}$ scintillator which are read out by wavelength shifting (WLS) fibers. The energy resolution of the electromagnetic calorimeter is approximately $16 \% / \sqrt{E}$ with a constant $1 \%$ term. At the depth of the electromagnetic shower maximum (approximately $6 X_{0}$ ), a position detector is installed which is made of scintillator strips read out by WLS fibers. These shower maximum chambers have provided effective identification of electrons and photons. The gap between wedge modules is covered by a tungsten bar (of $12 X_{0}$ length) backed by a wire chamber; in this way, some of the response of particles that would otherwise be lost is recovered.

The hadron calorimeter is an iron/scintillator sampling device; its unit layer consists of $5 \mathrm{~cm}$ iron and $6 \mathrm{~mm}$ scintillator. To reduce sensitivity to radiation, naphthalene

\footnotetext{
${ }^{3}$ The radiation length is the mean distance over which a high energy electron's energy is reduced to $1 / \mathrm{e}$ of its original energy
} 


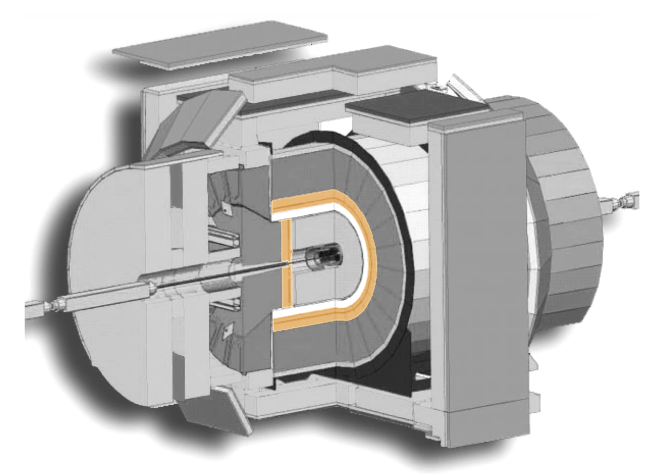

(a) Location of the electromagnetic calorimeter in the CDF detector

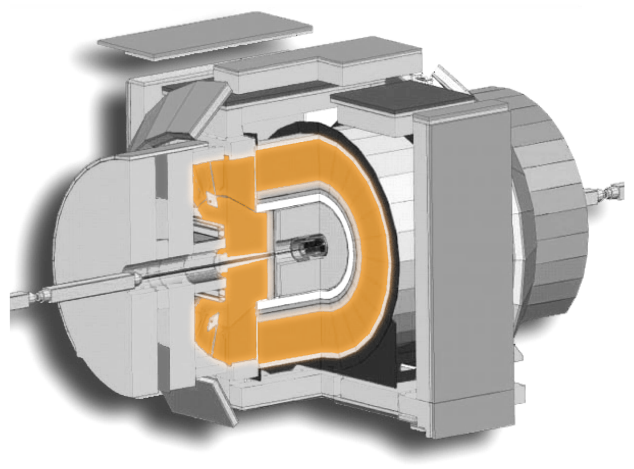

(b) Location of the hadronic calorimeter in the CDF detector

Figure 10: Location of the electromagnetic (a) and hadronic (b) calorimeters in the CDF II detector

scintillators are used. The hadronic calorimeter consists of 23 unit layers just like the electromagnetic calorimeter. The coverage is extended to $3^{\circ}$ by stainless steel disks which are attached to the inner $10^{\circ}$ cone.

Both sections have the same tower segmentation and both have stereo shower maximum detectors as well as pre-shower capability. Each tower is 15 degrees in azimuth by about 0.11 in pseudorapidity.

\subsubsection{The Muon Detectors [6][9]}

The outermost layer of the CDF detector is the muon detector, which comprises four systems of scintillators and proportional chambers over the region $|\eta| \leq 2.0$. There are different absorbers, namely the calorimeter steel, the magnet return yoke, additional steel walls, and the steel from the forward muon toroids.

CMU: The Central Muon Detector (CMU) provides coverage of $|\eta|<0.6$ using drift tubes with a maximum drift time of $800 \mathrm{~ns}$. It is located outside the calorimeter, whose steel acts as a filter. The CMU's basic unit is a rectangular drift cell of $6.35 \times 2.68 \times 226$ $\mathrm{cm}$ with a $60 \mu \mathrm{m}$ stainless steel wire in the center which are operated in proportional mode. Sixteen of these cells form a module of which there are 144 in the CMU. For each muon, the location is reconstructed in both $\phi$ and $z$.

CMP: The second part of the muon detector, the Central Muon Upgrade (CMP), is located behind not only the calorimeter steel but it is also shielded by an additional $60 \mathrm{~cm}$ of steel. It is situated in the region $55^{\circ} \leq \theta \leq 90^{\circ}$. Its rectangular cells cover a fixed length in $z$ but vary in their pseudorapidity coverage. The cells have a maximum drift time of $1.4 \mu \mathrm{s}$ and are run in proprotional mode. They are arranged in four layers. On the outside surface of the wall drift chambers a layer of scintillation counters (called CSP) is installed. 


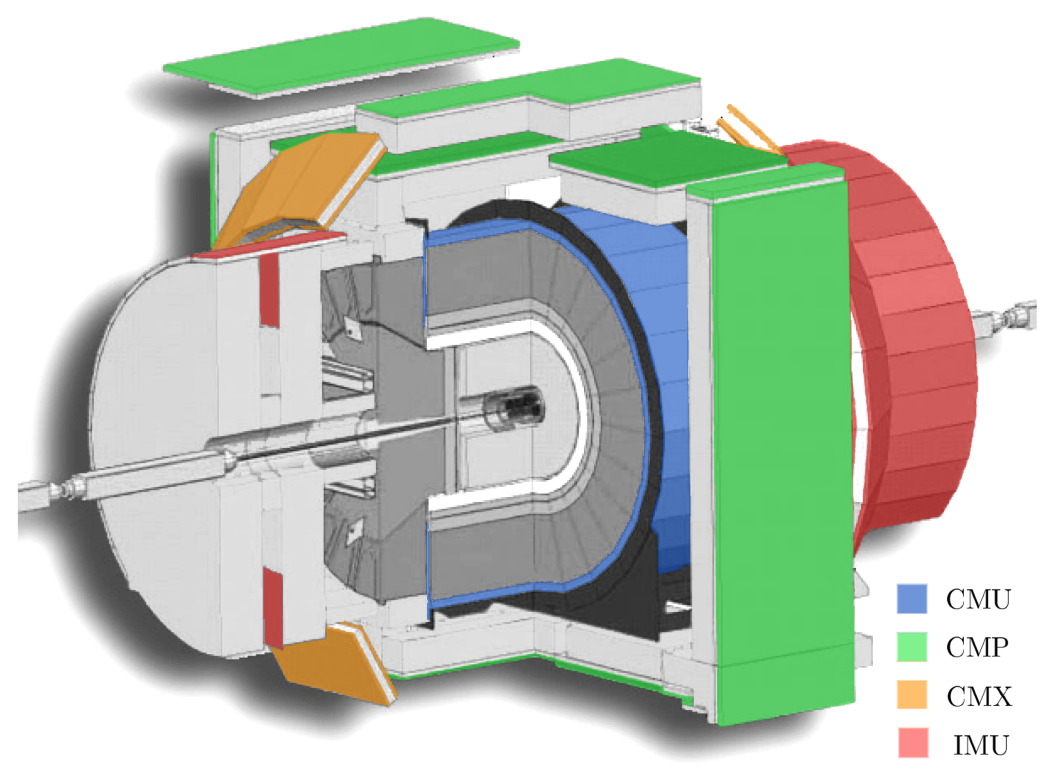

Figure 11: Illustration of the location of the muon detector system's components

CMX: The third part of the muon detector is the Central Muon Extension (CMX) which is made up of conical sections of drift tubes and scintillation counters. It covers the region $0.6 \leq|\eta| \leq 1.0$ and is also shielded by the calorimeter steel. There is a $30^{\circ}$ gap at the top of the detector for the Tevatron Main Ring and the solenoid refrigerator. Furthermore, another azimuthal gap of $90^{\circ}$ is located at the bottom of the detector where the detector is interrupted by the floor. The drift tubes are, with the exception of length, just like those of the CMP. They are rectangular cells arranged in two physical layers for each logical layer with an overlap that is larger at the inner edge for geometrical reasons. The overlap provides redundancy as well as the measurement of the polar angle of the track.

IMU: The Intermediate Muon System (IMU) consists of a barrel of muon chambers (BMU) and scintillation counters (BSU) parallel to the beam axis and a ring-shaped array of scintillators (TSU) which are mounted perpendicular to the beamline. The BMU is made up of rectangular scintilllators, the Toroid Scintillators (TSU) of trapezoidal polystyrene scintillators. The whole IMU covers the region $1.0 \leq|\eta| \leq 2.0$, wherein the granularity is fine for $1.0 \leq|\eta| \leq 1.5$ and a bit less for $1.5 \leq|\eta| \leq 2.0$ but still sufficient to identify isolated high $p_{T}$ tracks as muons or hadrons. The corresponding $p_{T}$ measurement are supplied by the tracking systems.

Trigger: The trigger consists of three levels that reduce the raw data rate of $7.6 \mathrm{MHz}$ to approximately $60 \mathrm{~Hz}$ which is stored:

- The first level trigger, a synchronous system with a $5 \mu$ s decision time, decides 
whether a given event is sufficiently interesting to be kept for further analysis by the second level. The rather long decision time of $5 \mu \mathrm{s}$ in comparison to the bunch crossing time made a pipelined approach necessary with an on-board buffer which temporarily stores 42 beam crossings (for the envisioned 132 ns bunch spacing). The muon trigger use the Extremely Fast Tracker (XFT) to find corresponding tracks from the COT for each bunch crossing. The track is then extrapolated (using $\phi$, transverse momentum $p_{T}$ and charge) to the track at the muon chamber radii and the following matching to muon stubs is performed in $\Delta \phi=2.5^{\circ}$ intervals.

- The second level trigger mainly refines the previous trigger level's cut on the trans-

verse momentum. It is an asynchronous system with an average decision time of $20 \mu \mathrm{s}$.

- The third level trigger is a processor farm where events are reconstructed and filtered using the full event information.

\subsection{Data Sample}

The integrated luminosity provided by the Tevatron is illustrated in Fig.12. The difference between the curve representing the delivered luminosity and the one representing the acquired luminosity can be explained by the time when the detector is turned off while the beam is still running. The rate at which data is taken has increased. Figure 13 shows the peak luminosity, which has also increased over the years and now routinely reaches $3 \times 10^{32} \mathrm{~cm}^{-2} \mathrm{~s}^{-1}$.

The events used in the analysis are compared to a "good run list", which is a double check to make sure that the whole detector was running properly when the events were recorded. The analysis uses a data sample corresponding to $5.7 \mathrm{fb}^{-1}$. 


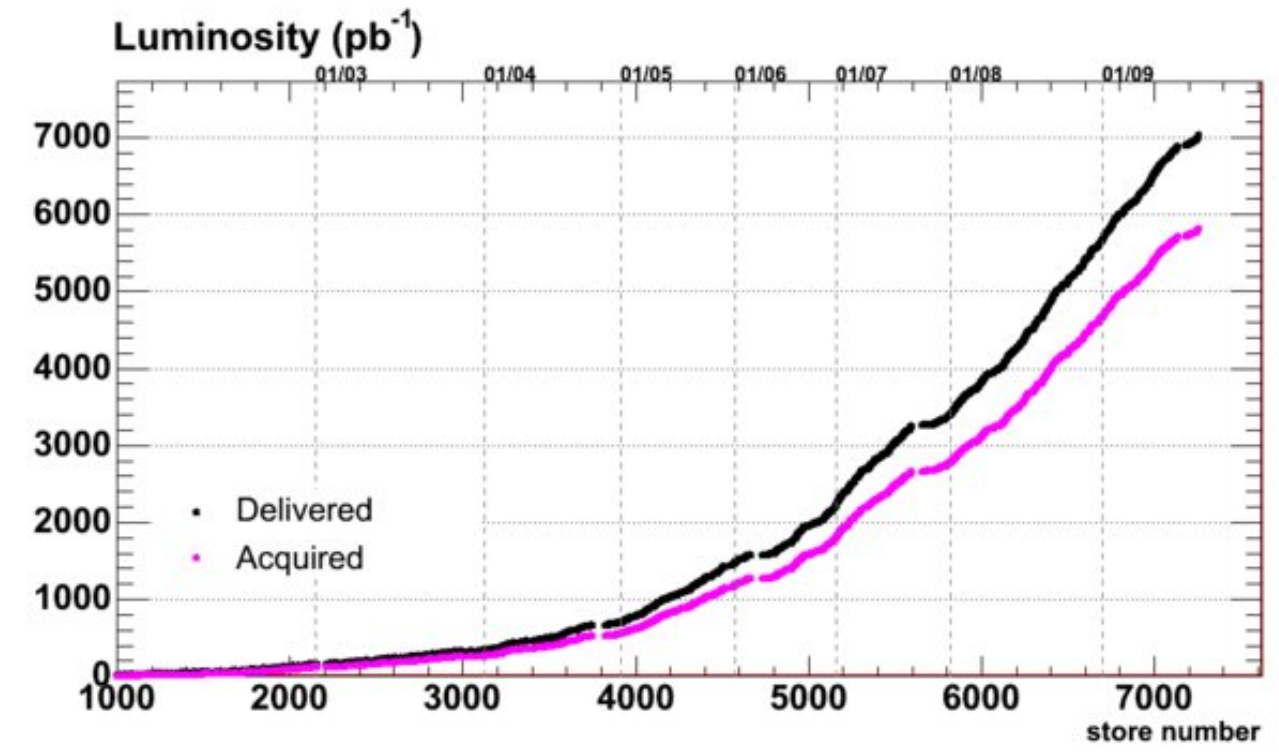

Figure 12: Integrated luminosity delivered by the Tevatron and recorded by CDF

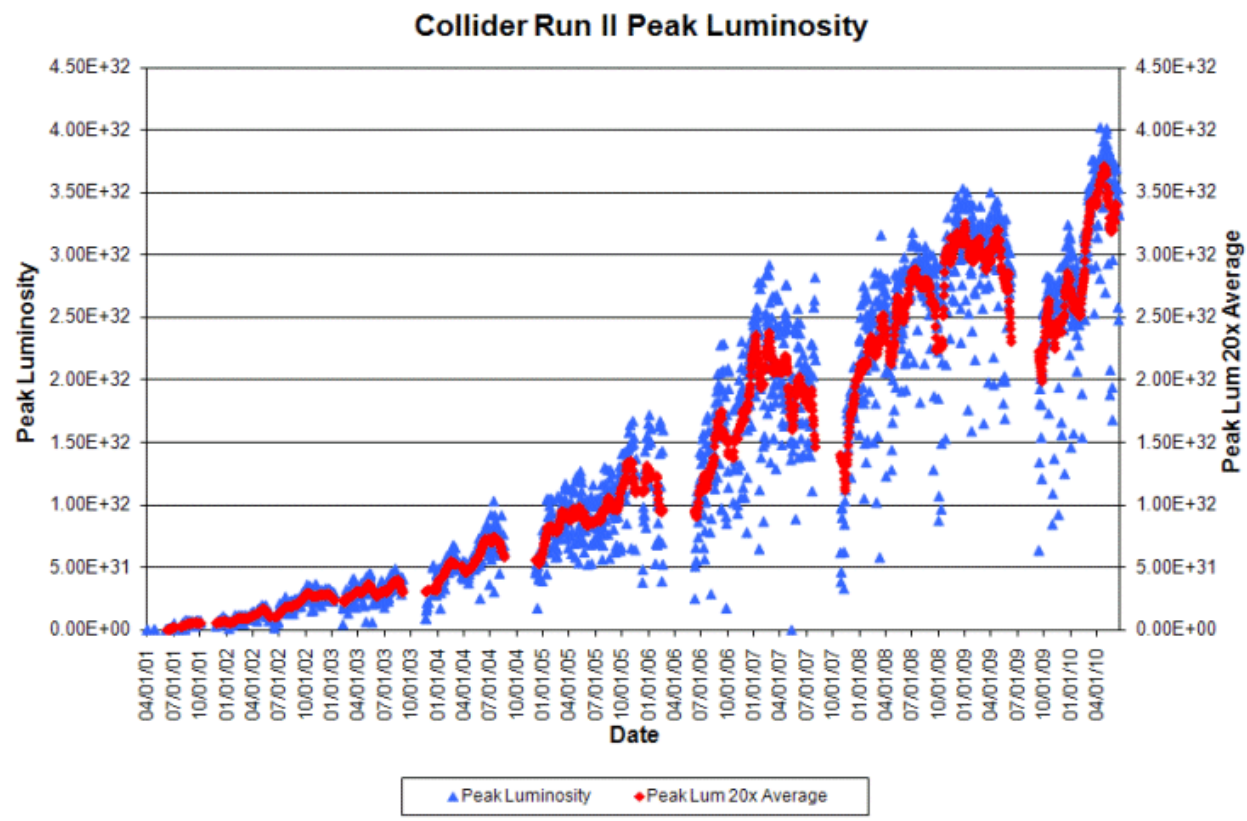

Figure 13: Peak instantaneous luminosity as a function of time 


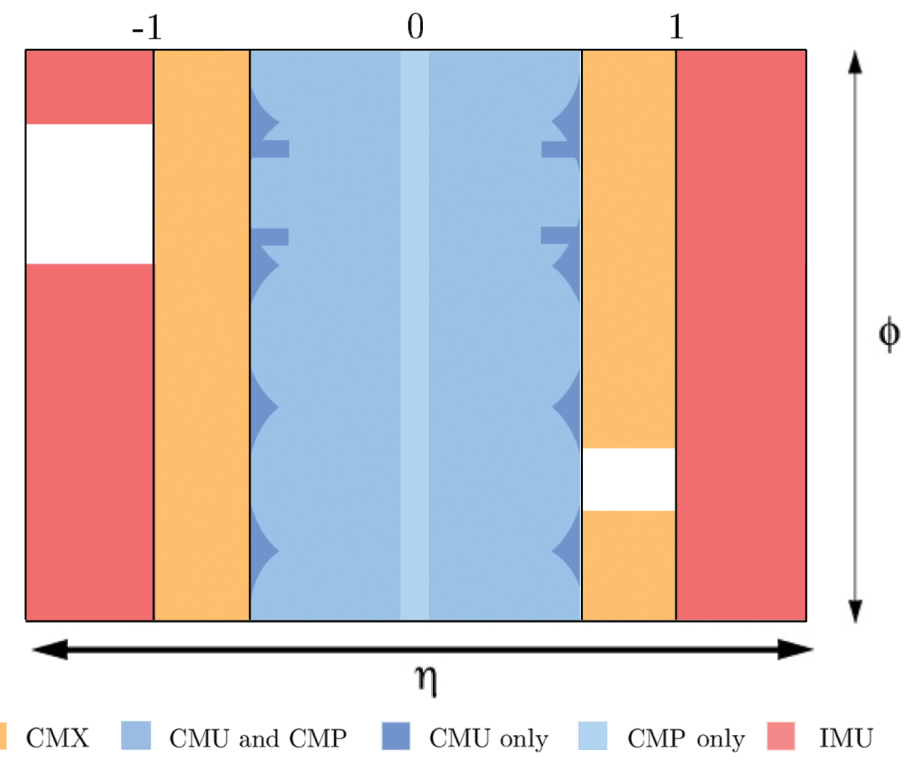

Figure 14: $\eta-\phi$ coverage of the muon detector parts. [16]

\section{New Categories}

Due to the inclusion of the phi gap trigger, changes in the categories were made necessary. In a first section, the coverage of $\eta-\phi$ space is explained; in the ensuing section, the definition of the phi gap trigger category is presented, followed by a section detailing its implementation in this analysis and the corresponding lepton category, along with the changes in other lepton categories made necessary by the new category.

\subsection{Coverage of $\eta-\phi$ Space}

The coverage of $\eta-\phi$ space was improved with the upgrade made from Run I to Run II, and several detector parts were added as previously mentioned in section 2.2.8. However, due to the different geometry of the CMU and CMP detector (see e.g. Fig 14), not all areas in $\eta-\phi$ space are covered by both detectors, leaving gaps in the coverage. The gaps in the CMU can be illustrated by considering muons that left a stub only in the CMP but not in the CMU, as shown in Fig. 15. One observes two distinct features:

- A central crack can be identified where the East and West Calorimeters meet

- There are thin periodic gaps, $2.5^{\circ}$ wide, every $15^{\circ}$

To see the relative importance of these two features, one can plot $\tilde{\phi}=\phi \bmod 15^{\circ}$ (see Fig. 16). The central crack contribution is only on the order of $30 \%$ and thus much smaller than that of the periodic gaps. Information about the track's $\phi$ angle is available at level 1 and 2, and in order to reduce the trigger rate, one requires the tracks to point 
at one of the gaps. Therefore, the CMP-only trigger only reclaims muons in the periodic gaps and not the central crack.

\subsection{CMP Identification Cuts}

The definition of the CMP category is based on the CMUP category; however, CMP muons are required not to have a stub in the CMU detector and only be fiducial to CMP. There is a $\pm 2^{\circ}$ cut around the gaps. The following requirements are imposed: [1]

- $E_{\text {em }} \leq 2+\max (0,(\mathrm{p}-100) \cdot 0.0115) \mathrm{GeV}$ where $E_{\text {em }}$ is the energy deposited in the electromagnetic calorimeter

- $E_{\text {had }} \leq 6+\max (0,(\mathrm{p}-100) \cdot 0.028) \mathrm{GeV}$ where $E_{\text {had }}$ is the energy deposited in the hadronic calorimeter

- Isolation $\leq 0.1$, where the isolation is the ratio between the transverse energy deposited in a cone of size $\Delta R<0.4$ around the lepton and the transverse energy of the lepton.

- $\left|z_{0}\right|<60 \mathrm{~cm}$ where $z_{0}$ is the origin of the track, measured with respect to the center of the detector (this cut ensures that the muon originates from close to the nominal beam crossing point)

- The muon must be fiducial to CMP, but not fiducial to CMU

- There must be at least 3 good COT axial segments (out of 5)

- There must be at least 2 good COT stereo segments (out of 5)

- The distance $\left|d_{0}\right|$ must be smaller than $0.2 \mathrm{~cm}$ or, if the number of hits in the Silicon detector is non-zero, $\left|d_{0}\right|<0.02 \mathrm{~cm}$.

- The distance $\Delta x$, which is the distance in the $r-\phi$ plane between the tracks extrapolated to the muon system and the stub in the muon system, must be $\Delta x \leq \max \left(6.0,150.0 / p_{T}\right) \mathrm{cm}$.

- $\chi^{2} /$ ndf must be less than 3 (less than 4 if run $\leq 186598$ )

- There are additional requirements on the $\mathrm{x}$-fiduciality $(<0 \mathrm{~cm})$ and z-fiduciality $(<-3 \mathrm{~cm})$

- As previously mentioned, one requires the $\phi$ gap muons to have $\phi \bmod 15^{\circ} \leq 2$ or $\geq 13^{\circ}$ (this cut is explained in the next section) 


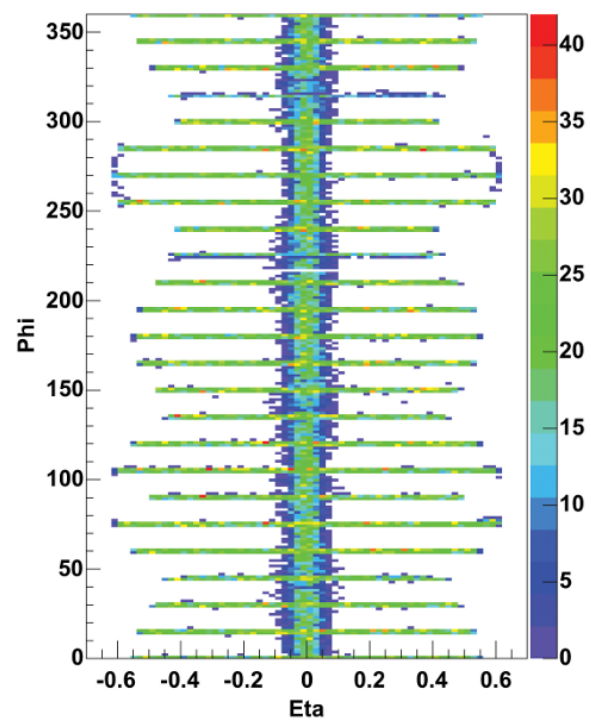

Figure 15: Illustration of the gaps of the CMU detector. In this $\phi-\eta$ scatter plot, only muons are placed that have stubs only in the CMP (hence called "CMP-only muons"), thereby illustrating the gaps of the CMU detector which lies inside. The $2.5^{\circ}$ gaps every $15^{\circ}$ are clearly visible as well as the central crack where the East and West Calorimeter modules meet (this gap is also illustrated in Fig 14. [1]

\subsection{Definitions of the New Categories}

In the present analysis, the phi-gap (CMP only) trigger is used, as laid out in the previous section, which requires the introduction of a new category and the adaptation of the previous extended muon category. The new category introduced for phi-gap trigger events is called "CMP":

\section{CMP category:}

- For data events, we require that the phi gap trigger be fired and that the angle within the $15^{\circ}$ interval be outside the $2^{\circ}<\tilde{\phi}<13^{\circ}$ window (where $\tilde{\phi}=$ $\left.\phi \bmod 15^{\circ}\right)$.

- For Monte Carlo, we require a hit in the CMP and the same condition for the angle is applied $\left(\tilde{\phi} \notin\left[2^{\circ}, 13^{\circ}\right]\right)$. No trigger turn on curve is applied.

The cut on $\tilde{\phi}$ is in place to ensure that the muons point at the CMU coverage gaps.

The addition of the CMP category required changes in the extended muon category to avoid double counting the new events. The extended muon category is also referred to as "loose category", though the events have to pass strict tight selection criteria (see for instance [22]). The previously used loose category contained events from all run periods, but since the phi gap trigger was commissioned in run period 10, the loose 


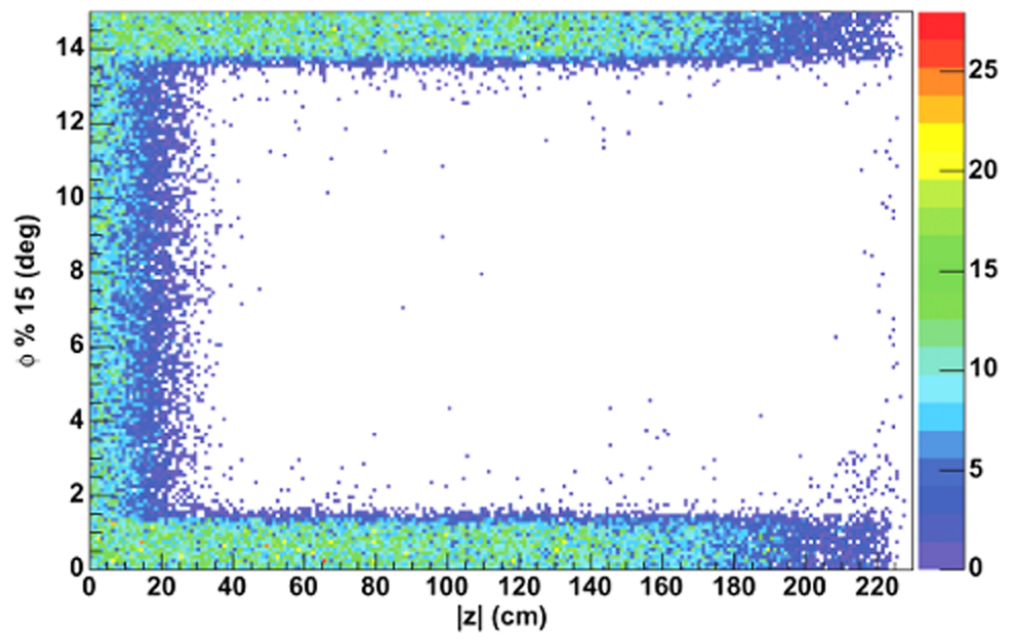

Figure 16: Scatter plot of CMP-only muons in $\tilde{\phi}-|z|$ space where $\tilde{\phi}=\phi \bmod 15^{\circ}$. The $\phi$ position is indicated as modulus $15^{\circ}$ to illustrate the relative weight of the central crack and the $\phi$ gaps. The overall central crack contribution is on the order of $30 \%$ and thus smaller than the overall contribution from the gaps.[1]

category was split up into a loose category before run period 10 (called "loose A") and a second loose category starting with run period 10 (called "loose B"); in the latter, CMP events were explicitly excluded to avoid double counting. The previous "loose" category is thus replaced by these two new categories:

\section{LOOSE A category}

- Data events must pass MET Jet L2 requirements and be from before period 10 .

- Monte Carlo must pass MET Jet L2 requirements; we apply a trigger turn on curve.

\section{LOOSE B category}

- Data events must pass MET Jet L2 requirements and be from period 10 or later

- Monte Carlo must pass MET Jet L2 requirements; we apply a trigger turn on curve.

- CMP events are explicitly excluded in both data and Monte Carlo

To estimate the gain achieved by the addition of the CMP data, one can compare the number of events from the MET + jets trigger (for more information about MET plus jet triggers, see [19]) and those from the new trigger; an overview of these numbers is given in Table 2. The CMU gap trigger can be added in the same way; as the data set grows, so will the gain from using these triggers as they will be included in a larger fraction of the data set. 


\begin{tabular}{|c|c|c|c|}
\hline selection & CMP (!firedMET) & MET (!firedCMP) & CMUP \\
\hline MET \& QCD & $1867(1191)$ & $1103(427)$ & 10060 \\
\hline pass L2 jets & & $570(214)$ & - \\
\hline
\end{tabular}

Table 2: Comparison of the number of events from the MET + jets trigger and the phi gap trigger in periods 10-23. In this run range, 1867 events will be added (corresponding to about $19 \%$ of CMUP), whereas we currently have some 570 events from the MET + jets trigger (corresponding to about $6 \%$ of CMUP). We thus gain about 1200 events. When cutting on $\tilde{\phi}$, an approximate $6 \%$ of the additional muons gets lost.

One can go one step further to compare the potential gain in Higgs events up to period 23, assuming $m_{H}=160 \mathrm{GeV}$ and without cutting on the dijet mass or vetoing events with a third jet with $E_{T}>12 \mathrm{GeV}$ but instead only those with $E_{T}>20 \mathrm{GeV}$ : While the previous loose category contained an anticipated 2.03 events, the new loose categories contain 1.46 anticipated events, and the CMP contains 2.10, thus leading to a total of 3.56 anticipated events. The gain of 1.53 events compared to the CMX with 7.91 events corresponds to about $19 \%$ of anticipated CMX Higgs events or approx. $10 \%$ of anticipated CMUP Higgs events. When comparing the gain to the total number of anticipated Higgs events, the improvement is $2.6 \%$.

When one considers the data periods when the phi gap trigger was active and includes all the listed cuts from Section 4, one finds for $m_{H}=160 \mathrm{GeV}$ a gain in the number of anticipated Higgs events corresponding to $23 \%$ of the CMX or $12 \%$ of the CMUP, and compared to the total number of anticipated Higgs events one still finds a gain of $3.1 \%$. For 150 and $170 \mathrm{GeV}$ the gain is slightly smaller but similar, while it is much smaller for $m_{H}=180-200 \mathrm{GeV}$. 


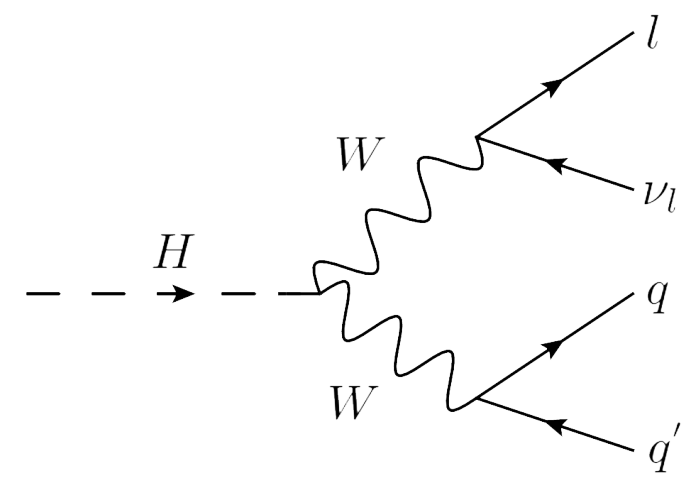

Figure 17: Leading order Feynman diagram of the signal process

\section{Event Selection}

Finding the Higgs particle requires a good understanding of the background processes involved to be able to identify the faint signal and ignore the background. The cross section of the Higgs production is very small and to complicate the matter further, the signal to background ratio of the analysis is tiny. It is thus crucial to make appropriate cuts to select only events relevant to the analysis and to have the best possible statistics.

The final state contains two $W$ bosons, one of which decays hadronically $\left(W \rightarrow q q^{\prime}\right)$ while the second decays leptonically $(W \rightarrow l \nu)$; the tree level Feynman diagram of the process is shown in Fig. 17. The branching ratio for a hadronic $W$ decay is approximately twice as large as that of a leptonic $W$ decay. For this analysis, it is required that one $\mathrm{W}$ decay hadronically to give two jets and the other decay leptonically to either an electron or a muon (which can be identified with high purity in the detector) with the corresponding neutrino. Since the tau lepton is difficult to detect, the practical leptonic branching ratio is a bit smaller. The neutrino escapes the detector but its transverse energy can be inferred from the missing transverse energy in the transverse plane.

In a first section, the major backgrounds are described including vetoes and cuts to reduce them. The ensuing section gives an overview of the trigger paths and the offline event selection.

\subsection{Backgrounds and Vetoes}

One of the major challenges of a Higgs boson search are the overwhelming backgrounds, which dominate by four orders of magnitude. By requiring two jets, a lepton and missing transverse energy $\left(\mathscr{E}_{T}\right)$, we select events that have the same signature as our signal; however, many background processes have the same or similar signatures. Some of those contributions can be reduced by event vetoes. 


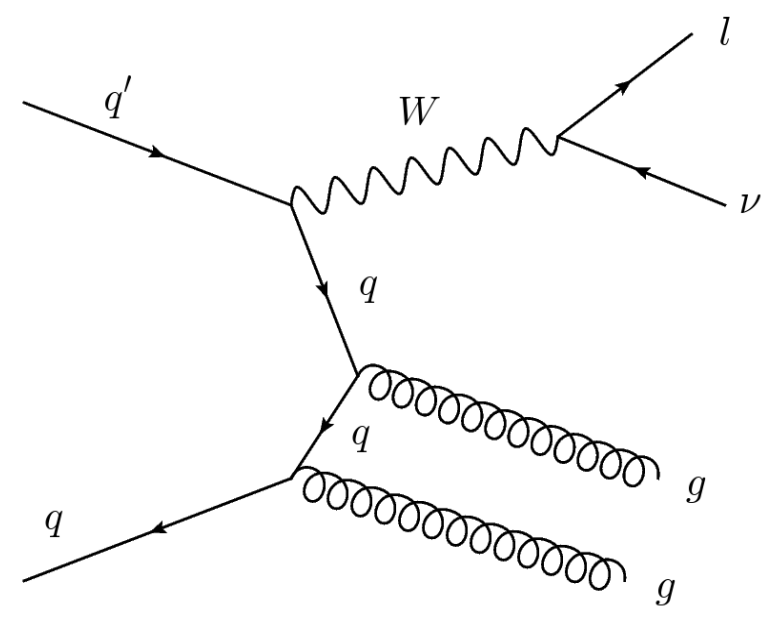

Figure 18: One of the contributing diagrams for the $W+$ jets background: A leptonically decaying $W$ is produced in association with two gluons

\subsection{1 $W+$ Jets}

The largest background contribution stems from $W+$ jets events; these come from quark interactions that radiate a $W$ boson in association with two jets, with the $W$ boson decaying leptonically $\left(W \rightarrow e \nu_{e}\right.$ or $\left.W \rightarrow \mu \nu_{\mu}\right)$. The tree level diagram of an example is given in Fig. 18. The final state signature is identical to that of $H \rightarrow W W$, yet with a much higher cross section. This background poses a challenge in many respects; it is very dominant (by four orders of magnitude more frequent than the signal), cannot be reduced by simple vetoes, and the modeling of this background results in significant systematic uncertainties in the measurement. There are some ways to reduce this background nonetheless, such as vetoing events with additional jets: events containing a third jet with $E_{T}>12 \mathrm{GeV}$ and $|\eta|<2.0$ are removed. Furthermore, we cut on the dijet mass as explained in section 4.1.7.

\subsubsection{Top Production}

A second background process is the top pair production, yet it is not as dominant as the $W+$ jets background. This decay is not problematic per se, since the final state signature is different from the signature one expects from $H \rightarrow W W$. However, the signature can be misinterpreted, for instance in the case of misidentification or failure to detect a particle (as illustrated in Fig. 19), such as when one of the leptons is missed or in lepton + jets decays in which two jets are not detected.

This background is reduced by vetoing events with a second lepton. The second lepton is identified as any tight lepton as well as an electron in the plug calorimeter or a nonisolated lepton in the CEM,CMUP or CMX. The veto on events with a third jet with $E_{T}>12 \mathrm{GeV}$ will strongly reduce the background from $t \bar{t}$ decays. 


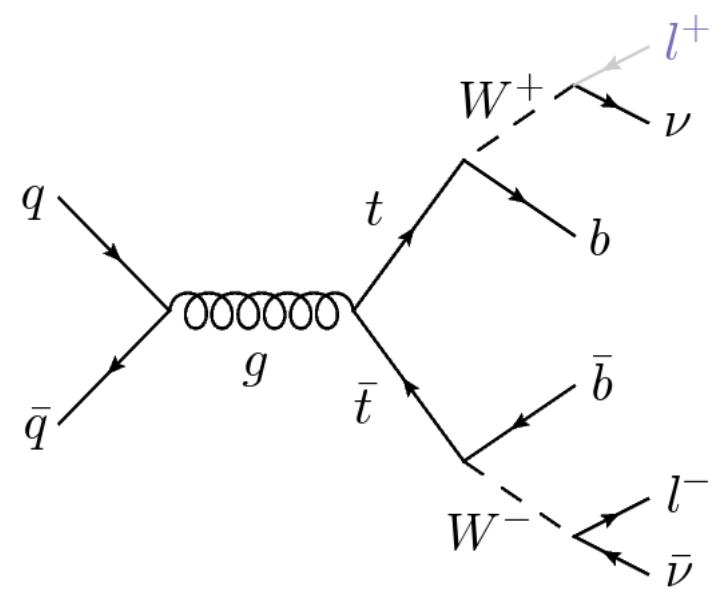

Figure 19: One of the contributing diagrams for the top production background: this event passes the event selection due to a failure to detect a lepton (grey particle)

\subsubsection{QCD Multijet}

A third background comes from events containing no $W$ bosons (also referred to as "non- $W$ " events); the respective inclusive cross section is considerable, yet to be confused with $H \rightarrow W W$ events one of the jets needs to "fake" a lepton and at the same time a mismeasurement needs to create a large missing transverse energy. An example of a QCD multijet background event is given in Fig 20. While the combined probability of these two cases is rather low, the corresponding high inclusive cross section leads to a non-neglible number of events. Additionally, the modeling of these events is rather challenging as they rarely pass all selection cuts. It is not possible to simulate these events with Monte Carlo, and one thus uses a data-based sample and estimates.

A special QCD veto is put in place to reduce this background (especially since it is hard to model). Since jets fake electrons more often than they fake muons, the veto is

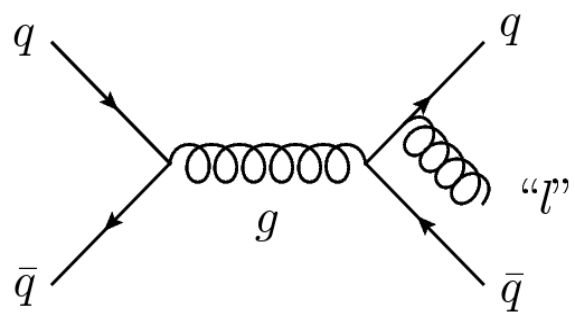

Figure 20: One of the contributing diagrams for the QCD multijet sample. In order to be accepted, a QCD event must have a jet from a quark or gluon pass all lepton selection requirements and be misidentified as a lepton. 


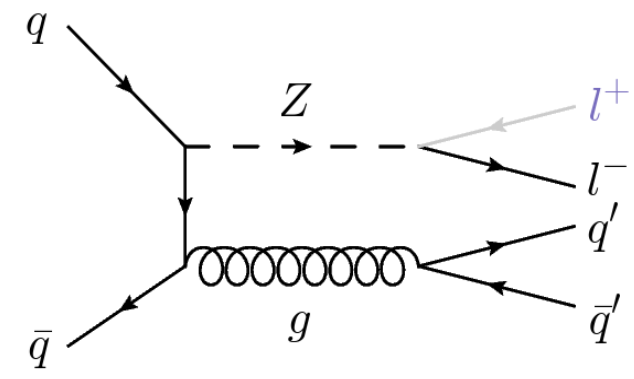

Figure 21: An example of a $Z+$ jets diagram having the same signature as the signal; in order to pass the event selection criteria, one of the leptons from the $Z$ boson decay must go undetected.

tighter for electron events. One can consider the transverse mass of the $W$,

$$
m_{T}(W)=\sqrt{2\left(p_{T l} p_{T \nu}-p_{x l} p_{x \nu}-p_{y l} p_{y \nu}\right)}
$$

and require it to be $m_{T}(W)>10 \mathrm{GeV}$ for muon events and $m_{T}(W)>20 \mathrm{GeV}$ for electron events. Furthermore, additional cuts are applied for electrons.

\subsubsection{Z+Jets}

The $Z+$ jets contribution is rather small, as $Z$ bosons decay to give either a pair of leptons or neutrinos and thus do not often "fake" an $H \rightarrow W W$ event. However, one needs to take into consideration the large inclusive cross section, which, when combined with the fact that in some events a lepton may be lost (and its energy is then considered missing energy), one still obtains a non-trivial remaining background. An example is shown in Fig. 21.

One thus uses a $Z$ boson veto, which works by looking for a second lepton in the event that fulfills looser electron and muon identification criteria than those described above for the first lepton in the event. The event is rejected if the second lepton has the opposite charge of the identified lepton in the event, and the invariant mass of the two leptons is close to the $Z$ boson mass (i.e. $76<m_{l l}<106 \mathrm{GeV}$ ).

\subsubsection{Diboson}

The last background considered in the analysis is the contribution from electroweak dibosons production $(W W, W Z$ and $Z Z)$ which also generates a small background, particularly $W W$ and $W Z$, which have the same final state signature as $H \rightarrow W W$. This background is relatively small compared to $W+$ jets.

\subsubsection{Cosmic Ray Veto}

Muons from cosmic rays may pass all muon identification criteria. One thus uses a cosmic ray veto which uses tracking and timing information; tracking is used to separate 

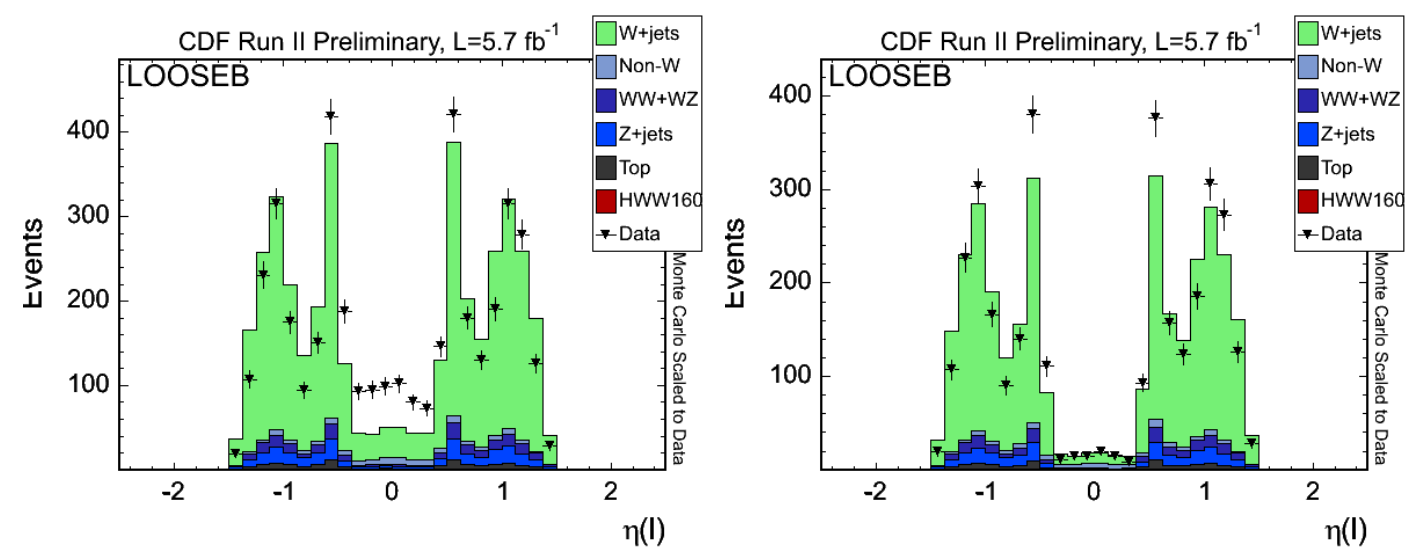

Figure 22: An excess of data events was found in the central region of the lepton $\eta$ in the LOOSEB category (illustrated on left); it was determined to be caused by the SCMIO which was consequently excluded (right).

\begin{tabular}{|l|c|c|c|c|c|c|}
\hline & $150 \mathrm{GeV}$ & $160 \mathrm{GeV}$ & $170 \mathrm{GeV}$ & $180 \mathrm{GeV}$ & $190 \mathrm{GeV}$ & $200 \mathrm{GeV}$ \\
\hline efficiency & $58.8 \%$ & $61.5 \%$ & $60.9 \%$ & $59.8 \%$ & $59.0 \%$ & $59.0 \%$ \\
\hline
\end{tabular}

Table 3: Efficiency of the adaptation of the veto (from $E_{T}<20 \mathrm{GeV}$ to $W_{T}<12 \mathrm{GeV}$ ) on events containing a third jet

particles passing through the detector from top to bottom as opposed to particles originating from a collision, with back-to-back tracks. Furthermore, timing information can also be used as cosmic ray events are generally not in time with collisions.

\subsubsection{Additional Cuts}

Mismodeling was observed in the lepton $\eta$ for the extended muon category starting in run period 10 (see Fig,22). This was caused by mismodeling in the SCMIO, which was not described well by the Monte Carlo; the SCMIO was thus excluded.

An additional lepton cut was introduced to take care of mismodeling in the event probabilities (the matrix element method is explained in Section 6): We require $\Delta \phi\left(E_{T}\right.$,lepton $)<3.0$ for leptons with $p_{T}>100 \mathrm{GeV}$. Additionally, third jets with $E_{T}>12 \mathrm{GeV}$ and $|\eta|<2.0$ are removed as mentioned previously. Originally, events with a third jet were only vetoed if the corresponding transverse energy was $E_{T}>20 \mathrm{GeV}$, but the modeling was suboptimal. These two cuts are motivated by the corresponding improvements in the event probability modeling as illustrated in Fig. 23. The efficiency of the lepton cut is above $99 \%$ and it affects backgrounds and signal about equally. The efficiency of the adaptation of the veto for the third jet, setting the threshold at $12 \mathrm{GeV}$ instead of $20 \mathrm{GeV}$, is on the order of $60 \%$ for the signal (varying as a function of the assumed Higgs mass, see Table 3 and $68 \%$ for the backgrounds. 

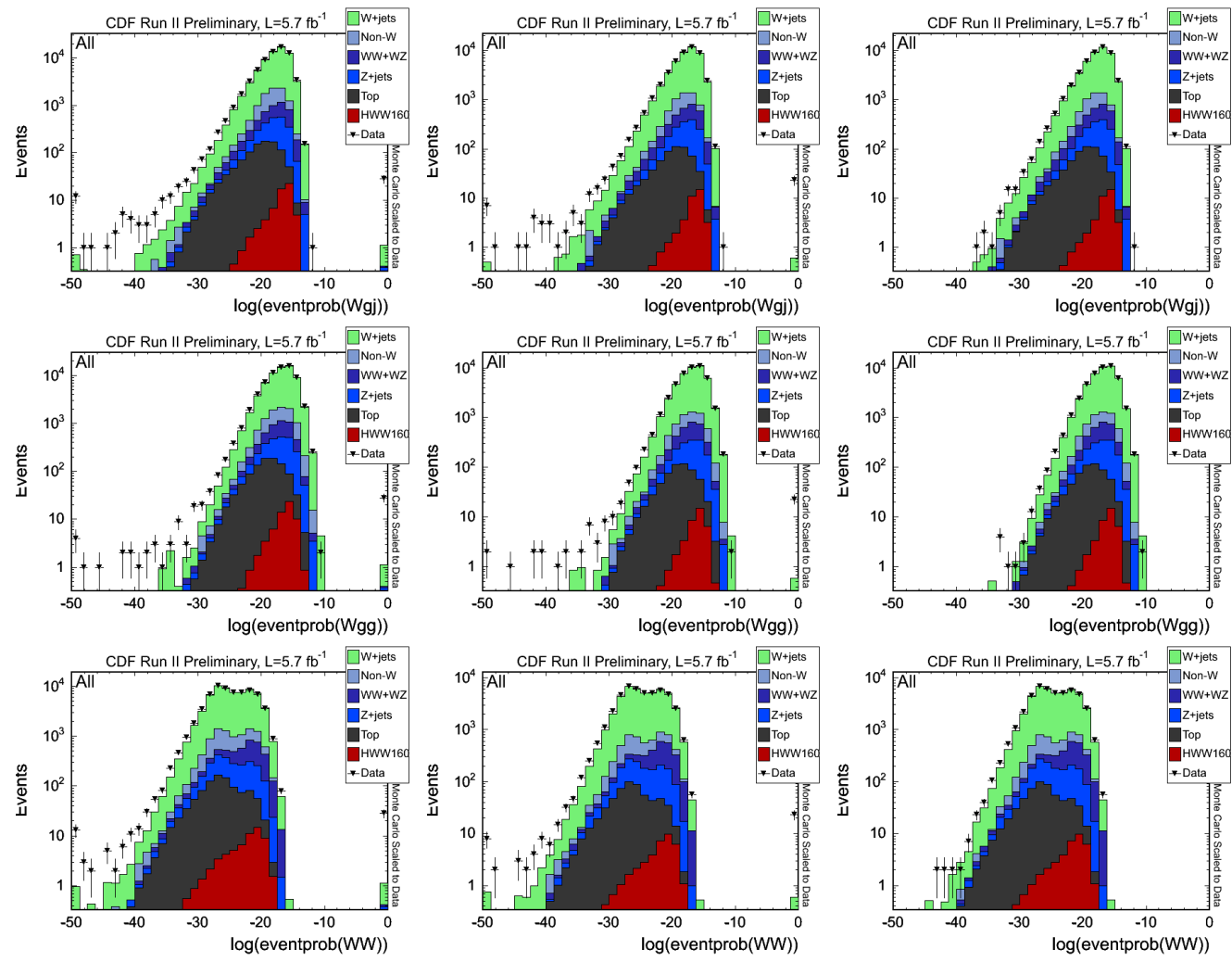

Figure 23: The event probability modeling is improved by introducing a lepton cut (lepton $p_{T}<100 \mathrm{GeV}$ or $\left.\Delta \phi\left(\mathbb{E}_{T}, l\right)<3.0\right)$ and rejecting events with a third jet with $E_{T}>12 \mathrm{GeV}$ and $|\eta|<2.0$. In the top row, the logarithm of the event probability for $W g j$ is shown, in the second row for $W g g$ and in the third $W W$; the first column contains neither lepton cut nor the cut on the third jet, the second column contains only the cut on the second jet and the third column contains both the cut on the third jet and the lepton cut. With the additional cuts the modeling overall improves significantly.

Furthermore, we require the dijet mass to be between $65<M_{j j}<95 \mathrm{GeV}$. The cut on the dijet mass was introduced to suppress more background and improve modeling as can be seen in Fig. 24. The efficiency of the dijet mass cut, along with the two previously discussed cuts, is $21 \%$ for the backgrounds and about $40 \%$ for the signals, again varying as a function of the assumed Higgs mass.

\subsection{Trigger Paths}

The events used in the analysis come from different trigger paths of which three trigger on high $p_{T}$ electrons and muons and a forth triggers on jets and missing transverse energy. The phi gap trigger has been described in Section 3.

- Central Electron Trigger: The CEM events come from the central electron trigger; at L1, those events are required to have a calorimetry tower with a transverse 

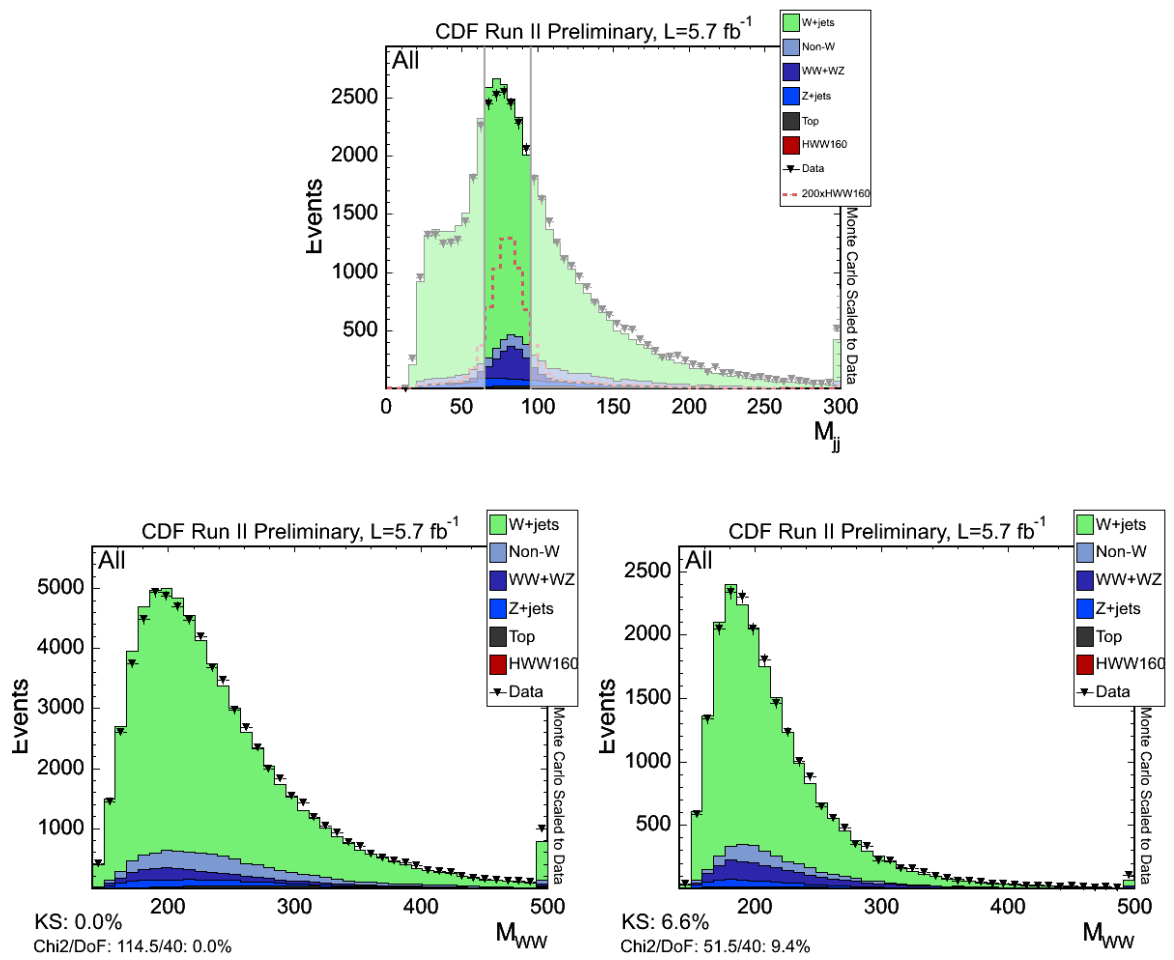

Figure 24: The cut on the dijet mass at $65 \mathrm{GeV}$ and $95 \mathrm{GeV}$ (illustrated above) improves the modeling for several variables, e.g. $M_{W W}$ (second row), and cuts away a large part of the background while preserving the important part of the signal. The cut also removes poorly modeled dijet mass regions.

energy in excess of $8 \mathrm{GeV}$ and $E_{h a d} / E_{e m}<0.125$, i.e. the ratio between hadronic energy and electromagnetic energy must be smaller than 0.125. At L2, a cluster is formed of several calorimeter towers, which is required to have $E_{T}>18 \mathrm{GeV}$. A shower profile is imposed at level 3. The energy threshold for electrons is raised to $20 \mathrm{GeV}$, which has only a small effect on the order of a few percent on the turn-on, which is small enough not to affect the analysis.

- Central muon trigger: The CMUP events come from the central muon trigger. At level one, these events are required to have stubs in both the CMU and the CMP detectors, corresponding to a muon with $p_{T}>6 \mathrm{GeV}$ and a matching track with $p_{T}>4 \mathrm{GeV}$. The calorimeter cluster at level two must correspond to a minimum ionizing particle, the $p_{T}$ track threshold is $15 \mathrm{GeV}$ and at level three, requirements are placed on the distance between a stub and the corresponding track extrapolated to the muon detectors.

- Central Muon Extension Trigger: Events that contain muons in the pseudorapidity range $0.6<|\eta|<1.0$ can be collected by the CMX trigger. The requirements are in general very similar to those used for the CMUP, however there is no second line of drift tubes which effectively means that there is a higher probability 


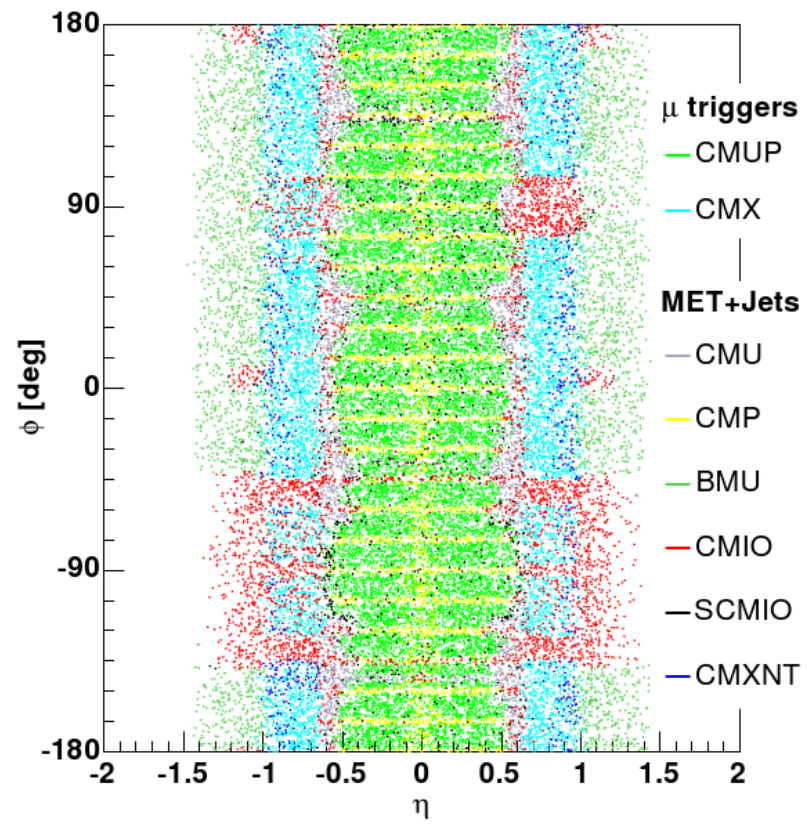

Figure 25: $\eta-\phi$ space coverage by the different subdetector systems

of jets faking muons. The CMX is prescaled, i.e. a given fraction of events is automatically rejected, so that the DAQ system can handle the data flow.

- $\mathbb{E}_{T}+$ jets trigger: When one considers the coverage of $\eta-\phi$ space obtained by using the CMUP and CMX triggers, one finds large gaps which can be filled by using an additional trigger which relies on two jets and large $E_{T}$ (referred to as $E_{T}+$ jets trigger). An illustration of the coverage of $\eta-\phi$ space by the different triggers is given in Fig. 25. The muon events are selected from this trigger offline. There are several requirements for events; at level one, events are required to have $E_{T}>15 \mathrm{GeV}$, where the $E_{T}$ is calculated based on calorimeter towers with $E_{T}>10 \mathrm{GeV}$ and two or more jets. At level three, the $E_{T}$ requirement is raised to $35 \mathrm{GeV}$. In recent data periods the trigger rates grew too large for data acquisition so that a new requirement that one jet needs to be central was imposed to reduce the rate. In order for the trigger to be fully efficient at level 2, the following offline requirements need to be fulfilled:

- The event must contain two jets, each having $E_{T}>25 \mathrm{GeV}$, and one jet being central

- The $\eta-\phi$ space distance between the two,

$$
R:=\sqrt{\left(\phi_{1}-\phi_{2}\right)^{2}+\left(\eta_{1}-\eta_{2}\right)^{2}}
$$

must be larger than 1.0 


\subsection{Offline Event Selection}

Events are required to contain one high quality electron or muon; the electron and muon identification is outlined in CDF note 10124 [17], the CMP category has been discussed in Section 3.3. The cut on the transverse energy of the jets was adapted to improve modeling; it is at $30 \mathrm{GeV}$ for the leading jet and $20 \mathrm{GeV}$ for the second jet. 


\section{Monte Carlo Modeling}

The present analysis employs a Matrix Element technique, as laid out in Section 6, which uses kinematic parameters to compute the matrix elements. It thus relies on accurate modeling of background and signal processes. In this chapter, the Monte Carlo samples used in the modeling are listed in the first section, followed by a section on background estimates. In a third section, the event yields are presented.The chapter closes with the modeling validation.

\subsection{Monte Carlo Samples}

The $H \rightarrow W W$ events as well as all background processes, except the QCD multi-jet background, are generated by using event generators. A list of the Monte Carlo samples used is given in Table 5. Alpgen samples with Pythia parton showering are used to model the $W+$ jets and $Z+$ jets backgrounds whereas Pythia is used to model the $W W$ and $W Z$ processes.

The QCD multijet background cannot be modeled using Monte Carlo, as explained in Section 4 and in more detail below in Section 5.2.2. Therefore, the non- $W$ background is modeled using data [27.

\subsection{Background Estimate}

The estimation of background levels is similar to the procedure used for the pretag sample in the single top search (see for instance CDF note 9185 [26]).

Assuming that the data sample consists of the backgrounds mentioned previously in Section 4.1, the Monte Carlo based processes are calculated by using measured or theoretical cross sections, the integrated luminosity of our dataset, and Monte Carlo derived efficiencies $(\varepsilon)$ to calculate each normalization. For MC based processes (such as electroweak, single top, and $t \bar{t}$ ) the normalization is given by

$$
N_{p \bar{p} \rightarrow X}=\sigma_{p \bar{p} \rightarrow X} \cdot \varepsilon \cdot \int d t \cdot \mathcal{L} .
$$

In a second step, the non- $W$ QCD fraction is evaluated. As previously mentioned in the context of backgrounds (Chapter 4), the QCD background is the least understood and most poorly predicted. A large uncertainty of $40 \%$ is thus assigned to the normalization of this process. In order to estimate the fraction of our sample stemming from QCD processes, we make a fit to the $E_{T}$ spectrum. The non- $W$ QCD fraction is then simply applied to the amount of data in our signal region to obtain the normalization.

$$
N_{Q C D}=f_{Q C D} \cdot N
$$

So far only not all contributions have been taken into consideration, there is still a remainder stemming from processes that are neither electroweak, QCD nor top. One thus subtracts the MC-based processes and the non- $W$ QCD from the data to obtain the remainder which is then considered the $W+$ jets contribution.

$$
N_{W+j e t s}=N \cdot\left(1-f_{Q C D}\right)-N_{e w k}-N_{t o p}
$$




\subsubsection{Based Background Estimate}

There are numerous contributions from electroweak processes, namely $W W, W Z, Z Z$, $Z+$ jets, $t \bar{t}$ and single top events. The indicated estimates are based on the theoretical or measured $(Z+$ jets $)$ cross sections shown in Table 5 , the integrated luminosity of our dataset, trigger efficiency, and an overall selection efficiency derived from Monte Carlo simulation of each process. The estimates for our sample are given by

$$
N_{p \bar{p} \rightarrow X}=\sigma_{p \bar{p} \rightarrow X} \cdot \varepsilon_{\text {event }} \cdot \int d t \cdot \mathcal{L}
$$

where $\sigma_{p \bar{p} \rightarrow X}$ is the theoretical or measured cross section, $\int d t \cdot \mathcal{L}$ is the total integrated luminosity used in this analysis, and $\varepsilon_{\text {event }}$ is the selection efficiency which is derived based on Monte Carlo.

\subsubsection{Non- $W$ Background Estimate}

Because of their high inclusive cross section, non- $W$ events can arise in spite of the low combined probability of a simultaneous mismeasurement leading to a large $E_{T}$ and a jet "faking" an electron or muon. The exact cross section is unknown since these events rarely pass event selection criteria so that one would thus have to generate large QCD samples to obtain a sufficiently large number of events. An additional obstacle is the fact that the mismeasurements are often caused by instrumental effects which would make it once again harder in detector simulations. We therefore use data for modeling the non- $W$ background.

The non- $W$ fraction is estimated by fitting the $E_{T}$ spectrum in data to a sum of background $E_{T}$ shapes. The fit of the $E_{T}$ distributions is carried out for CEM, CMUP, CMX, LOOSE A, LOOSE B, and CMP categories; the fit includes the region below the analysis $E_{T}$ cut, which is enriched in QCD events.

The fit has one fixed component (from the MC-based processes) and two templates whose normalizations can float. The two floating templates are a Monte Carlo $W+$ jets template and a non- $W$ template, which is different depending on the lepton category. Three different types of samples are used:

- Anti-electrons: The first type comes from the central electron trigger; these events must fulfill the same kinematic requirements as tight electrons, but must fail at least two of the five cuts related to shower shapes.

- Jet electrons: These events pass a $20 \mathrm{GeV}$ jet trigger, with one jet resembling an electron with transverse energy $E_{T}>20 \mathrm{GeV}$ and they must have deposited $80-90 \%$ of their energy in the electromagnetic calorimeter. Furthermore, the jet also needs to have at least four tracks, thereby removing events that may have a real electron.

- Non-isolated muons: The third type of events comes from the central muon trigger and contain a muon; these events pass all identification requirements, but fail the isolation requirement. 
The anti-electron sample is used to model the shape of the non- $\mathrm{W}$ contribution in the CEM, CMUP, CMX, CMP, and the extended muon categories. Furthermore, the non-iso sample is used for the extended categories (but not the CMP).

The fits are shown in Figure 26, they are generally good. The arrow indicates the $E_{T}$ cut applied in the analysis, and the $f_{Q C D}$ value gives the percentage of QCD expected after applying the $\mathscr{E}_{T}$ cut. The templates are fitted to the $E_{T}$ distribution of data events using a binned likelihood fitter before the $\mathscr{E}_{T}$ cut has been imposed.
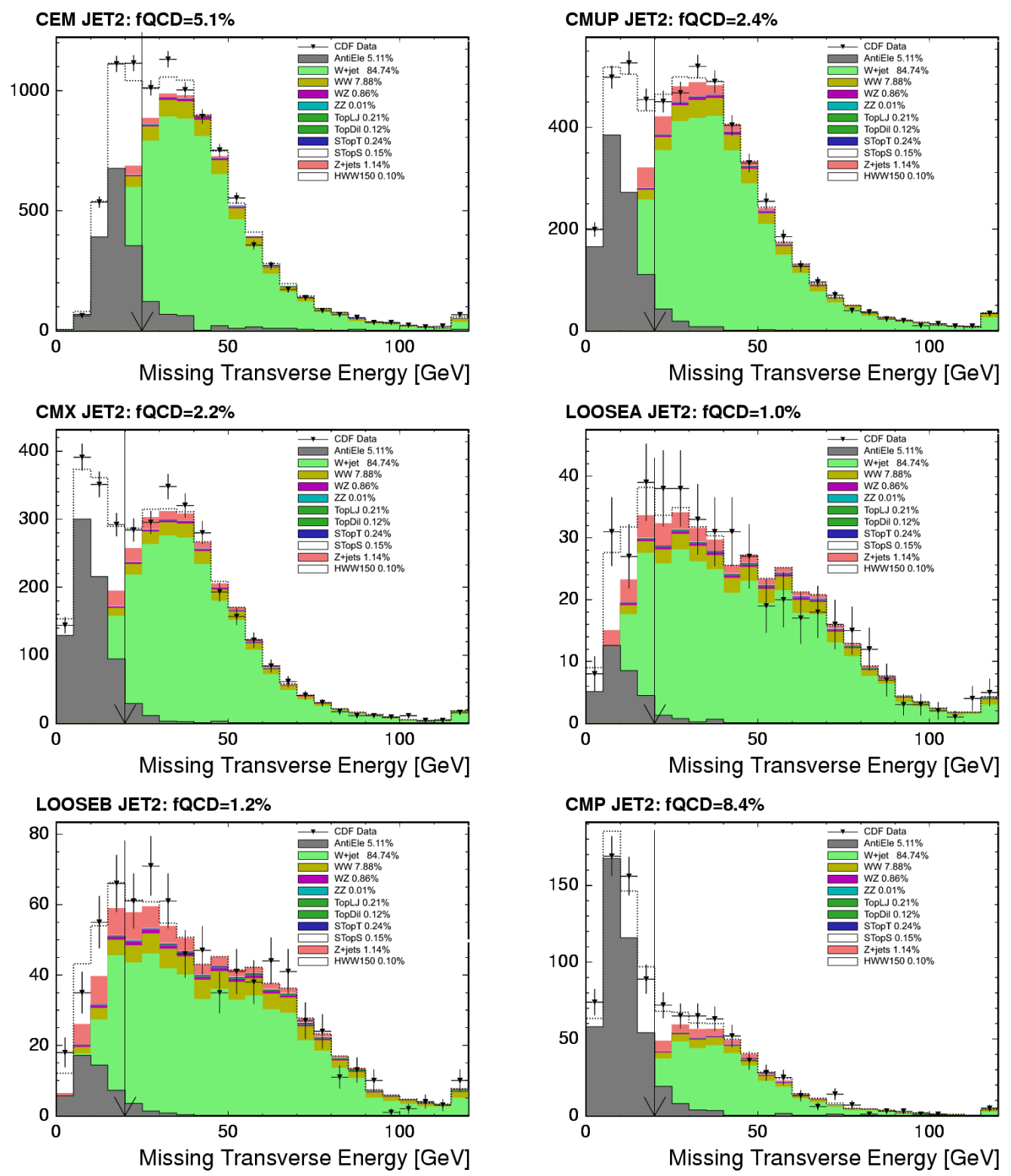

Figure 26: Missing $\mathrm{E}_{T}$ fit to determine non-W normalization in the CEM (top left), CMUP (top right), CMX (second row left), CMUP (second row right), LOOSE A (third row left), and LOOSE B (third row right). 
Once the QCD fraction $f_{Q C D}$ is calculated the number of QCD multi-jet events is simply:

$$
N_{Q C D}=f_{Q C D} \cdot N
$$

where $N$ is the total number of events.

\subsubsection{Signal Estimate}

The signal samples were generated for $150<m_{H}<200 \mathrm{GeV}$ in $10 \mathrm{GeV}$ increments using PYTHIA. The expected number of signal events is estimated at each Higgs boson mass point,

$$
N_{g g \rightarrow H \rightarrow W W}=\sigma_{g g \rightarrow H} \cdot \mathcal{B R}(H \rightarrow W W) \cdot \varepsilon_{\text {event }} \cdot \int d t \cdot \mathcal{L}
$$

where $\sigma_{g g \rightarrow X}$ and $\mathcal{B R}(H \rightarrow W W)$ for each Higgs mass are given in Table 4 , and the other terms were explained in Section 5.2.1.

\begin{tabular}{|c|c|c|}
\hline$m_{H}$ & $\sigma(g g \rightarrow H)$ & $\mathrm{BR}(H \rightarrow W W)$ \\
\hline $150 \mathrm{GeV}$ & 0.548 & 0.682 \\
\hline $160 \mathrm{GeV}$ & 0.439 & 0.901 \\
\hline $170 \mathrm{GeV}$ & 0.349 & 0.965 \\
\hline $180 \mathrm{GeV}$ & 0.283 & 0.935 \\
\hline $190 \mathrm{GeV}$ & 0.231 & 0.776 \\
\hline $200 \mathrm{GeV}$ & 0.192 & 0.735 \\
\hline
\end{tabular}

Table 4: Production cross section and branching ratios at the investigated Higgs mass points 


\begin{tabular}{|c|c|c|c|c|}
\hline Process & $\begin{array}{l}\text { Monte } \\
\text { Carlo }\end{array}$ & Sample (LL) & $\begin{array}{l}\text { Sample } \\
\text { (HL) }\end{array}$ & Cross section \\
\hline$H W W$ & Pythia & $\begin{array}{ll}\text { ehht1k } & (150), \\
\text { ehht1m } & (160), \\
\text { ehht1o } & (170), \\
\text { ehht1q } & (180), \\
\text { ehht1s } & (190), \\
\text { ehht1u } & (200)\end{array}$ & & $\begin{array}{l}0.548 \pm 0.027 \mathrm{pb}(150 \mathrm{GeV}) \\
0.439 \pm 0.022 \mathrm{pb}(160 \mathrm{GeV}), \\
0.349 \pm 0.017 \mathrm{pb}(170 \mathrm{GeV}) \\
0.283 \pm 0.014 \mathrm{pb}(180 \mathrm{GeV}) \\
0.231 \pm 0.012 \mathrm{pb}(190 \mathrm{GeV}) \\
0.192 \pm 0.010 \mathrm{pb}(200 \mathrm{GeV})\end{array}$ \\
\hline $\begin{array}{l}(W \rightarrow e \nu)+\text { jets } \\
(W \rightarrow \mu \nu)+\text { jets } \\
(W \rightarrow \tau \nu)+\text { jets }\end{array}$ & $\begin{array}{l}\text { Alpgen }+ \\
\text { Pythia } \\
\\
\begin{array}{l}\text { Alpgen }+ \\
\text { Pythia }\end{array} \\
\\
\text { Alpgen + } \\
\text { Pythia }\end{array}$ & $\begin{array}{l}\text { ptopw0, } \\
\text { ptopw1, } \\
\text { ptop2w, } \\
\text { ptop3w, } \\
\text { ptop4w } \\
\text { ptopw5, } \\
\text { ptopw6, } \\
\text { ptop7w, } \\
\text { ptop8w, } \\
\text { ptop9w } \\
\text { utopw0, } \\
\text { utop1w, } \\
\text { utop2w, } \\
\text { utop3w, } \\
\text { utop4w }\end{array}$ & $\begin{array}{l}\text { wtoph5, } \\
\text { wtoph6, } \\
\text { wtoph7, } \\
\text { wtoph8, } \\
\text { wtoph9 } \\
\text { wtophf, } \\
\text { wtophg, } \\
\text { wtophh, } \\
\text { wtophi, } \\
\text { wtophj } \\
\text { wtophp, } \\
\text { wtophq, } \\
\text { wtophr, } \\
\text { wtophs, } \\
\text { wtopht }\end{array}$ & From data fit \\
\hline $\begin{array}{l}(Z \rightarrow e e)+\text { jets } \\
(Z \rightarrow \mu \mu)+\text { jets } \\
(Z \rightarrow \tau \tau)+\text { jets }\end{array}$ & $\begin{array}{l}\text { Alpgen }+ \\
\text { Pythia } \\
\\
\text { Alpgen } \\
\text { Pythia } \\
\\
\text { Alpgen + } \\
\text { Pythia }\end{array}$ & $\begin{array}{l}\text { ztopp0, } \\
\text { ztopp1, } \\
\text { ztop2p, } \\
\text { ztop3p, ztop4p } \\
\text { ztopp5, } \\
\text { ztopp6, } \\
\text { ztop7p, } \\
\text { ztop8p, ztop9p } \\
\text { ztopt3, ztopt4, } \\
\text { ztopt2 }\end{array}$ & & $787 \pm 85 \mathrm{pb}$ \\
\hline Non- $W$ & Data & $\begin{array}{l}\text { Anti-electron, } \\
\text { jet-electron, } \\
\text { non-iso muons }\end{array}$ & & From $\not_{T}$ fit \\
\hline$\overline{t \bar{t}}$ & Pythia & $\begin{array}{l}\text { ttop75, } \\
\text { ttop75_1fb }\end{array}$ & & $6.7 \pm 0.8 \mathrm{pb}$ \\
\hline Single top & $\begin{array}{l}\text { Madevent }+ \\
\text { Pythia }\end{array}$ & $\begin{array}{l}\text { stop00, } \\
\text { stopm0, } \\
\text { stop20, stop2m }\end{array}$ & & $2.864 \mathrm{pb}$ \\
\hline$\overline{W W}$ & Pythia & ihht1a & & $11.66 \pm 0.7 \mathrm{pb}$ \\
\hline$W Z$ & Pythia & jhht1a & & $3.46 \pm 0.3 \mathrm{pb}$ \\
\hline$Z Z$ & Pythia & khht1a & & $1.51 \pm 0.2 \mathrm{pb}$ \\
\hline
\end{tabular}

Table 5: Monte Carlo samples used. 


\subsection{Event Yields}

Table 6 gives an overview of the expected event yields for all processes and the observed number of events. The lepton categories are divided into four columns in the table; when calculating the limits, the lepton categories in the first three columns are treated as one category, whereas LOOSE A and B (in Table 6 the last column) are treated as a second, separate category. The observed and predicted yields match well since the prediction is derived from a fit to the data.

\begin{tabular}{|l|c|c|c|c|}
\hline & CEM & CMUP +CMX & CMP & LOOSE \\
\hline HWW150 & $7.02 \pm 0.55$ & $6.6 \pm 0.4$ & $0.62 \pm 0.1$ & $0.36 \pm 0.05$ \\
\hline HWW160 & $13.85 \pm 1.08$ & $12.21 \pm 0.8$ & $1.14 \pm 0.19$ & $0.36 \pm 0.05$ \\
\hline HWW170 & $13.45 \pm 1.05$ & $11.46 \pm 0.7$ & $1.09 \pm 0.18$ & $0.46 \pm 0.06$ \\
\hline HWW180 & $10.4 \pm 0.81$ & $9.06 \pm 0.6$ & $0.87 \pm 0.14$ & $0.95 \pm 0.12$ \\
\hline HWW190 & $6.99 \pm 0.55$ & $6.05 \pm 0.4$ & $0.59 \pm 0.1$ & $1.24 \pm 0.16$ \\
\hline HWW200 & $5.68 \pm 0.44$ & $4.92 \pm 0.3$ & $0.48 \pm 0.08$ & $1.42 \pm 0.18$ \\
\hline Non-W & $341.2 \pm 147.7$ & $137.6 \pm 66.4$ & $38.5 \pm 21.2$ & $10.6 \pm 24.1$ \\
\hline Diboson & $581.8 \pm 44.9$ & $508 \pm 31.6$ & $48.5 \pm 7.2$ & $114.7 \pm 13.1$ \\
\hline top & $47.2 \pm 3.4$ & $39.5 \pm 2.2$ & $4.1 \pm 0.4$ & $15.5 \pm 1.2$ \\
\hline Z+jets & $75.1 \pm 9.3$ & $239.6 \pm 22.9$ & $32.9 \pm 6.2$ & $53.6 \pm 7.9$ \\
\hline W & $5576.3 \pm 147.7$ & $4915 \pm 66.4$ & $331.3 \pm 21.2$ & $731 \pm 24.1$ \\
\hline Data & 6679 & 5890 & 460 & 930 \\
\hline
\end{tabular}

Table 6: Expected and observed number of events

\subsection{Modeling Validation}

Once the background level prediction has been obtained, the next step consists of checking various kinematic distributions for the agreement between Monte Carlo and the data. Several distributions were checked and are given in Figures 27 to 38 . The relative contributions are taken from Table 6, but the sum of the Monte Carlo contribution is normalized to the data; the plots are thus shape comparisons only.

The eight plots show the distributions in the different categories, and the sum over them. The PHX category is included in the plots to illustrate the reasoning for its exclusion; it is not included in the sum drawn on the last canvas of each plot. The plotted variables are rather basic kinematic variables that enter the matrix element calculation; they include the energy (Fig. 27 and Fig. 29) and $\eta$ (Fig. 28 and Fig. 30 of the jets, as well as the lepton's momentum (Fig. 31) and $\eta$ (Fig. 32); furthermore, plots are included for the missing transverse energy (Fig. 33) as well as the transverse mass of the $W$ (Fig. 34), even though they do not enter the matrix element calculations. Those plots validate our understanding of the non- $W$ modeling. In the last plots, we check the kinematics of the dijet system in order to validate the modeling of the correlation between two jets. The angle between the two jets, $\Delta \phi=\left|\phi_{j_{1}}-\phi_{j_{2}}\right|$ (Fig. 36) as well as the $\eta-\phi$-space distance $\Delta R=\sqrt{\left(\eta_{j_{1}}-\eta_{j_{2}}\right)^{2}+\left(\phi_{j_{1}}-\phi_{j_{2}}\right)^{2}}$ (Fig. 35), the dijet mass 
$m_{j j}=\sqrt{\left(E_{j_{1}}+E_{j_{2}}\right)^{2}-\left(\overrightarrow{\left.p_{j_{1}}+\overrightarrow{p_{j_{2}}}\right)^{2}}\right.}$ (Fig. 38 and the transverse momentum of the dijet system (Fig. 37) are analyzed.

In general, the modeling is in very good agreement with the data. The non- $W$ modeling seems adequate, as the corresponding kinematic variables, such as the transverse momentum and the transverse energy as well as the transverse mass of the leptonic $W$, seem to be well modeled. At the same time, not all variables are optimally modeled. While the leading jet's transverse energy is well modeled (Fig. 27), the second jet's transverse energy is not (Fig. 29). The $\eta$ of both jets is well modeled (Fig. 28 and Fig. 30p, though again for the first jet the agreement is better. There is some mismodeling as well in the dijet system, which manifests itself for instance in the $\eta-\phi$-space distance between the two jets (35). 

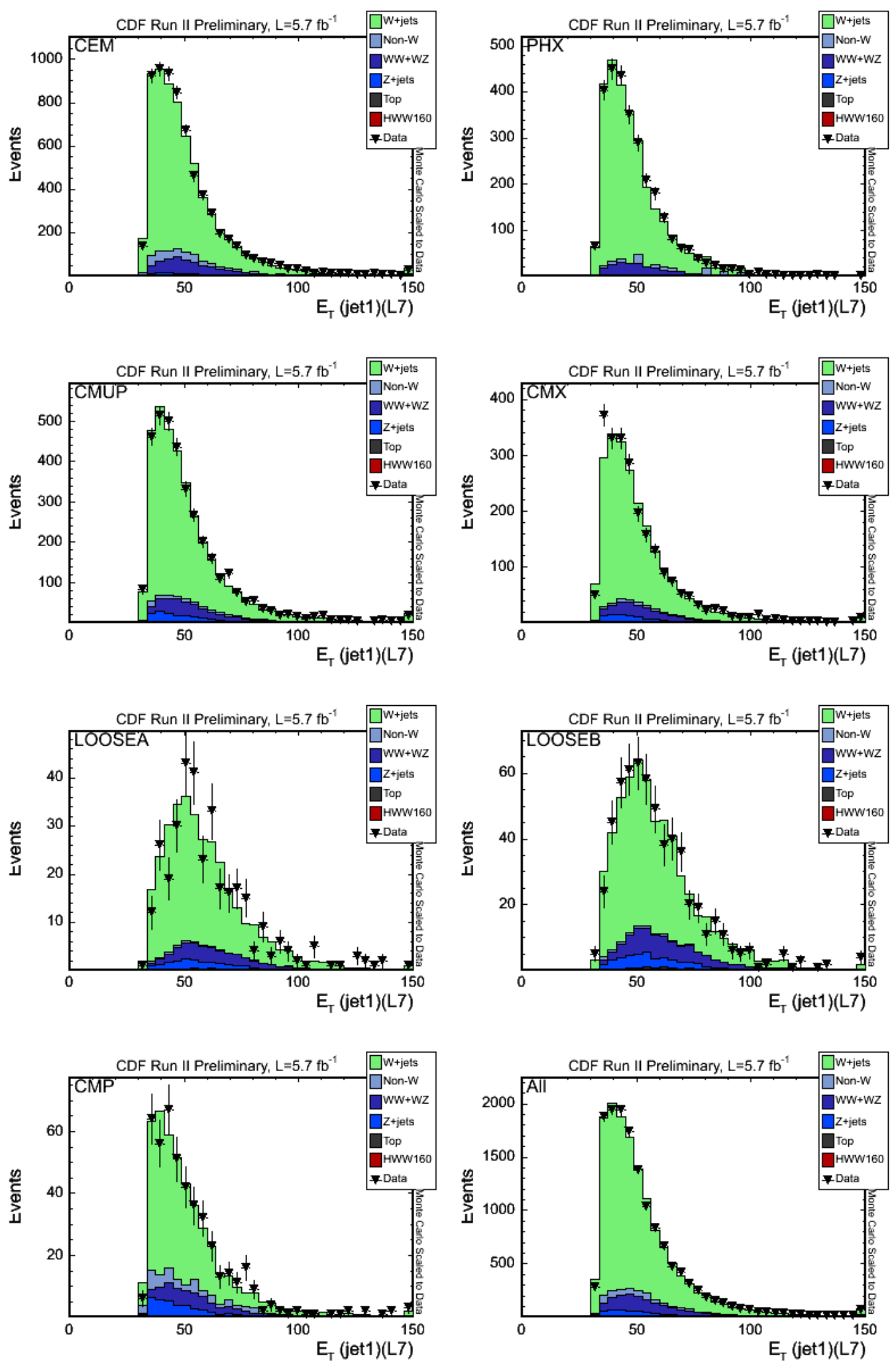

Figure 27: $E_{T}$ of the higher- $E_{T}$ jet 

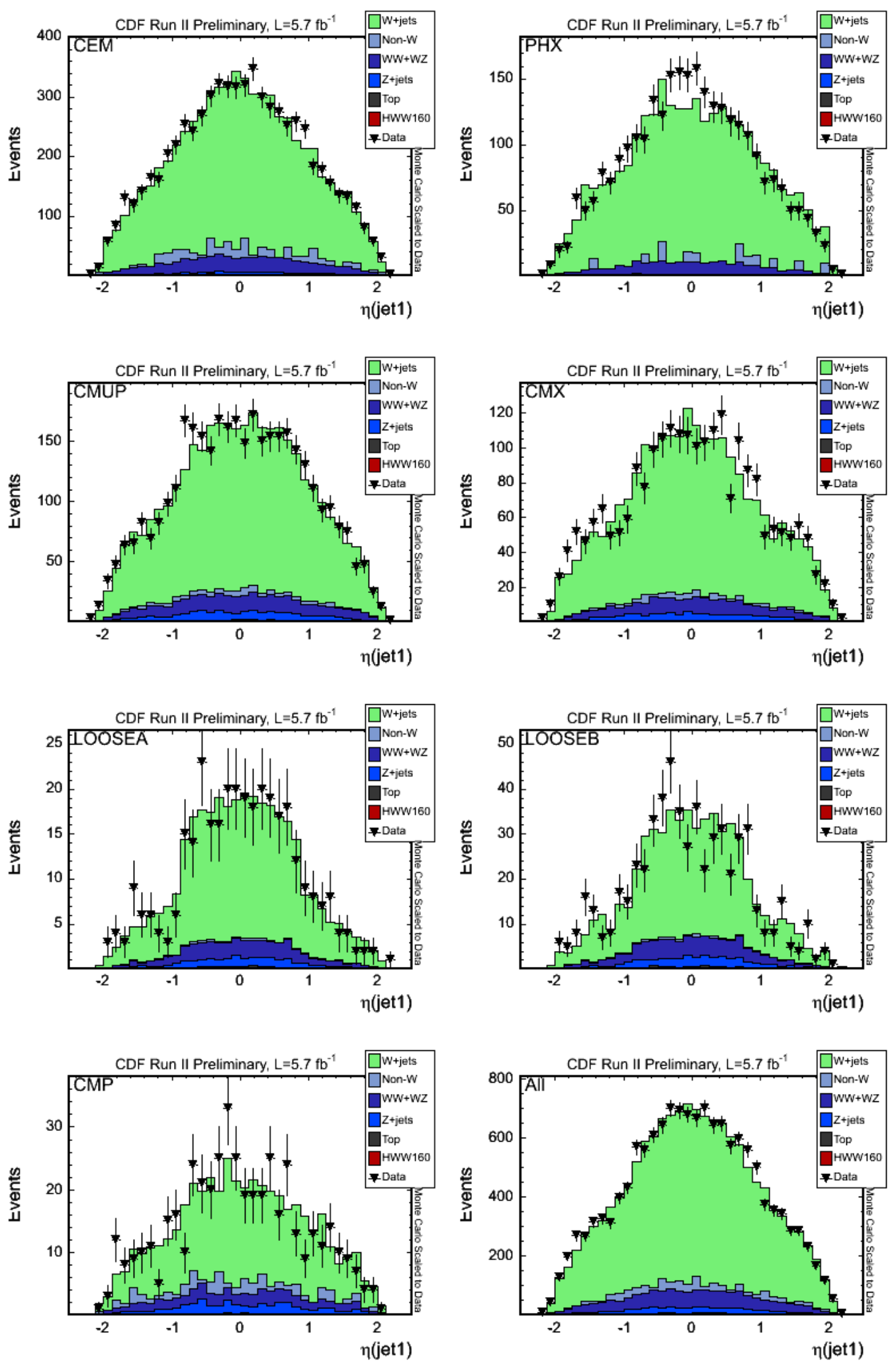

Figure 28: $\eta$ of the higher- $E_{T}$ jet 

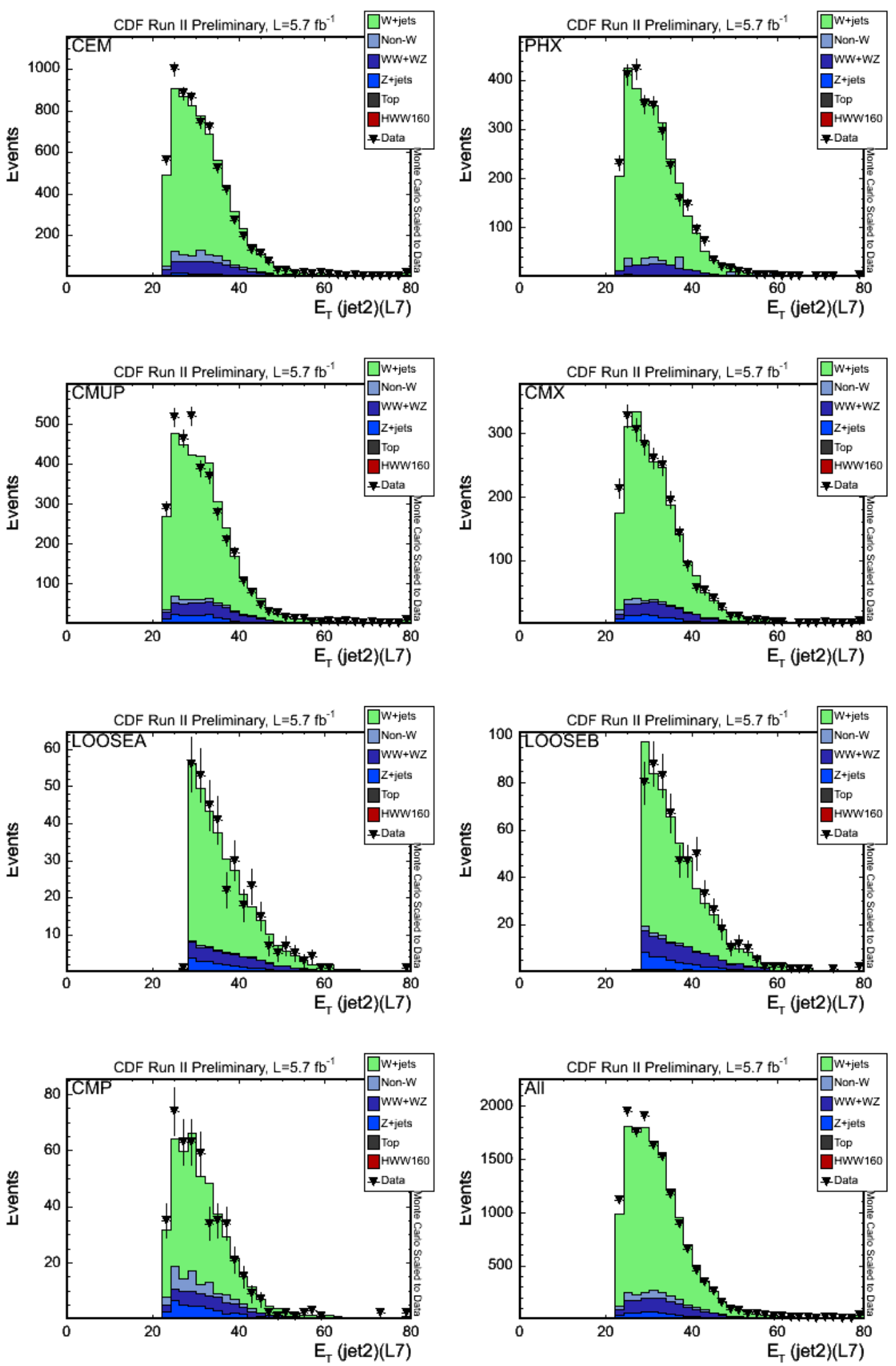

Figure 29: $E_{T}$ of the lower- $E_{T}$ jet 

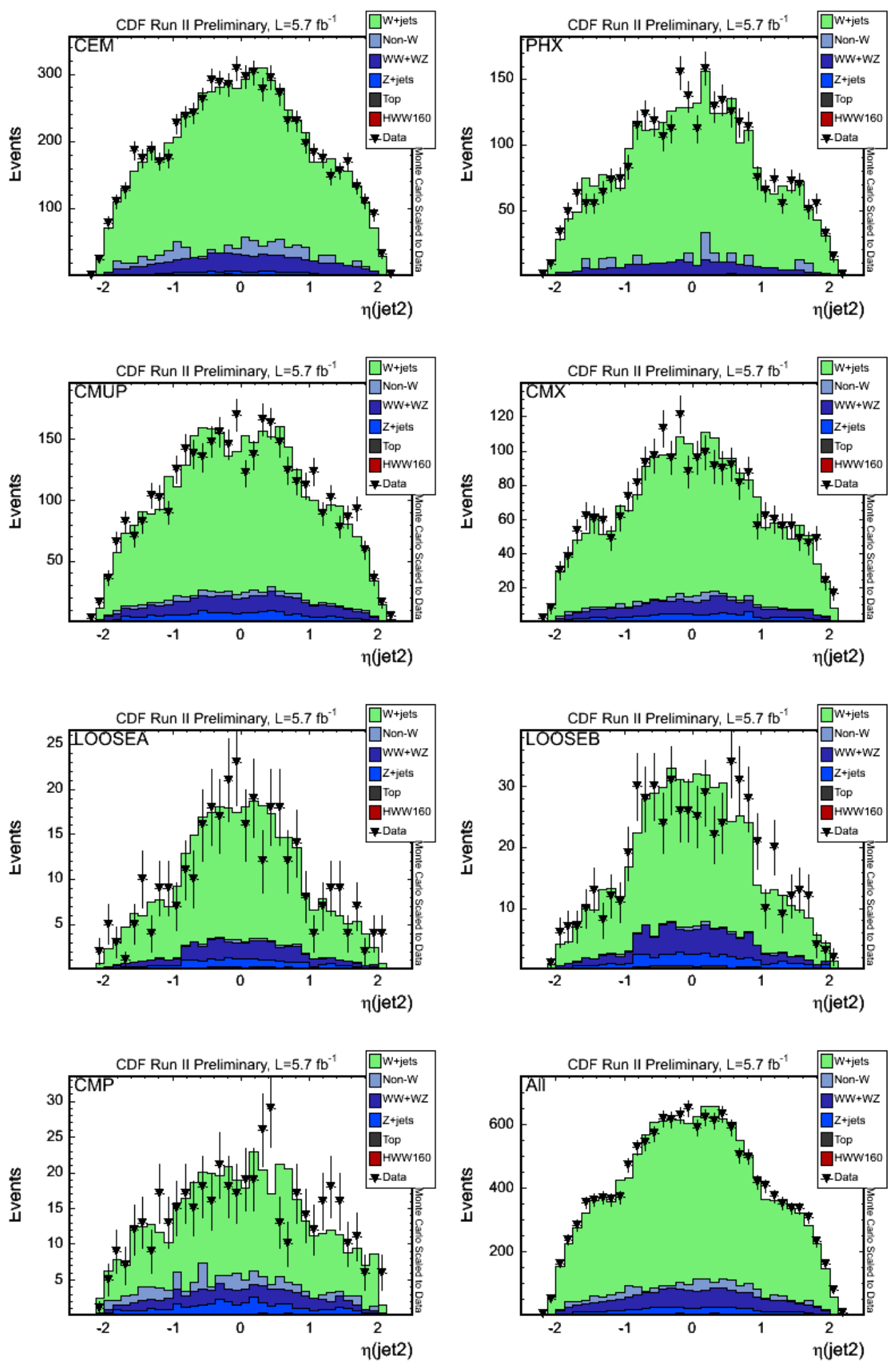

Figure 30: $\eta$ of the lower- $E_{T}$ jet 

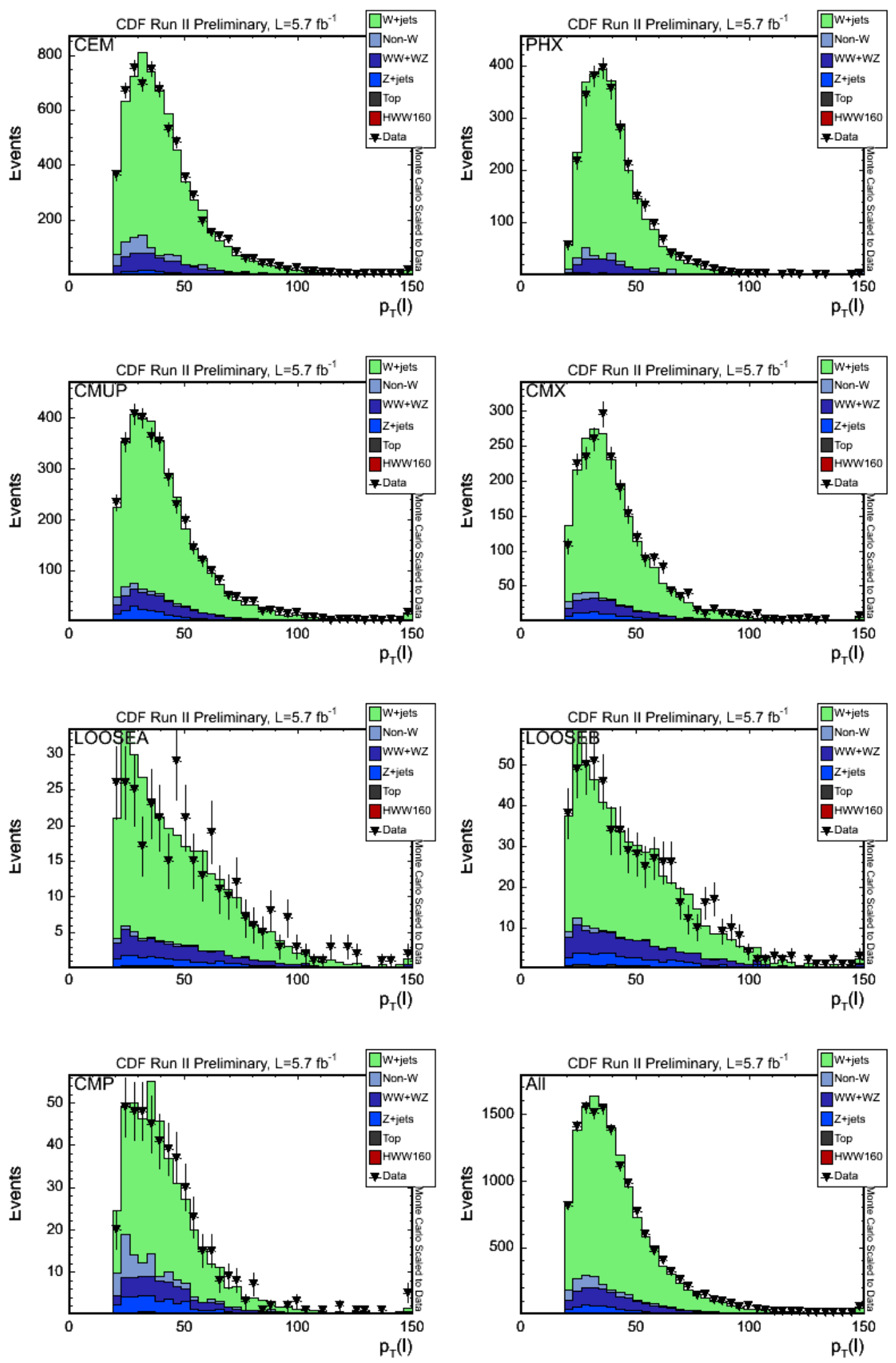

Figure 31: $p_{T}$ of the lepton. 

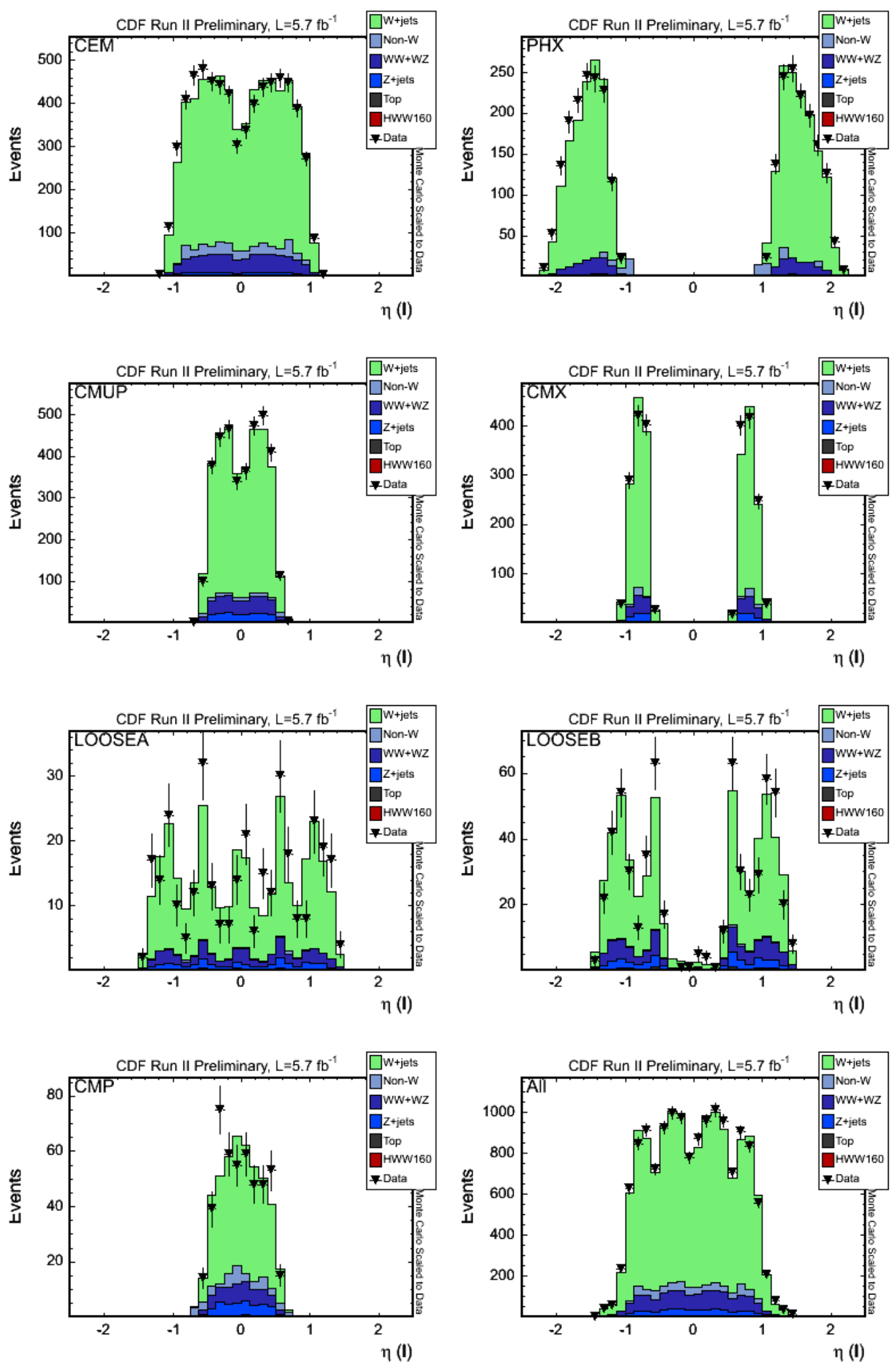

Figure 32: $\eta$ of the lepton. 

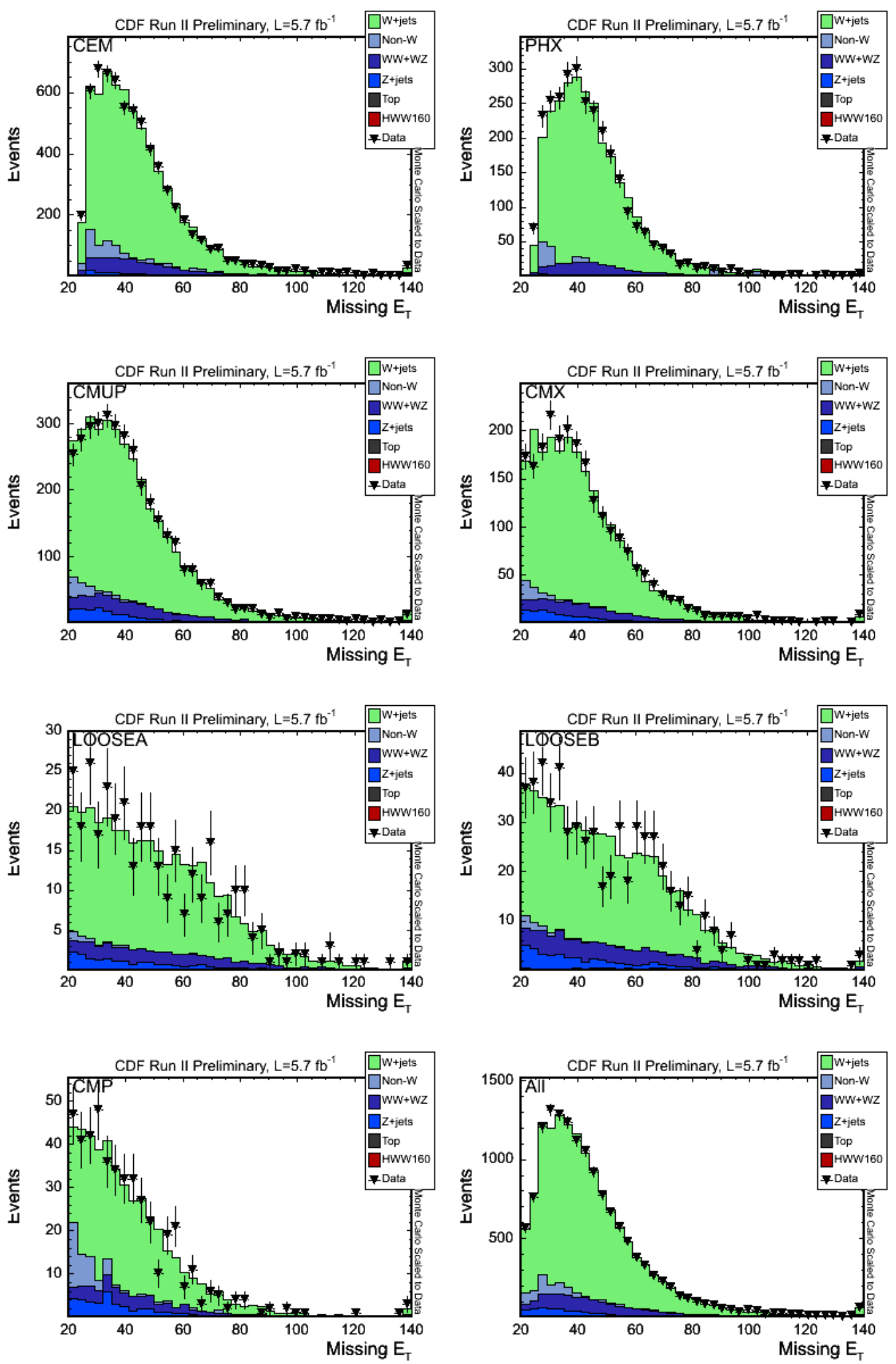

Figure 33: Missing transverse energy $\left(\not_{T}\right)$ 

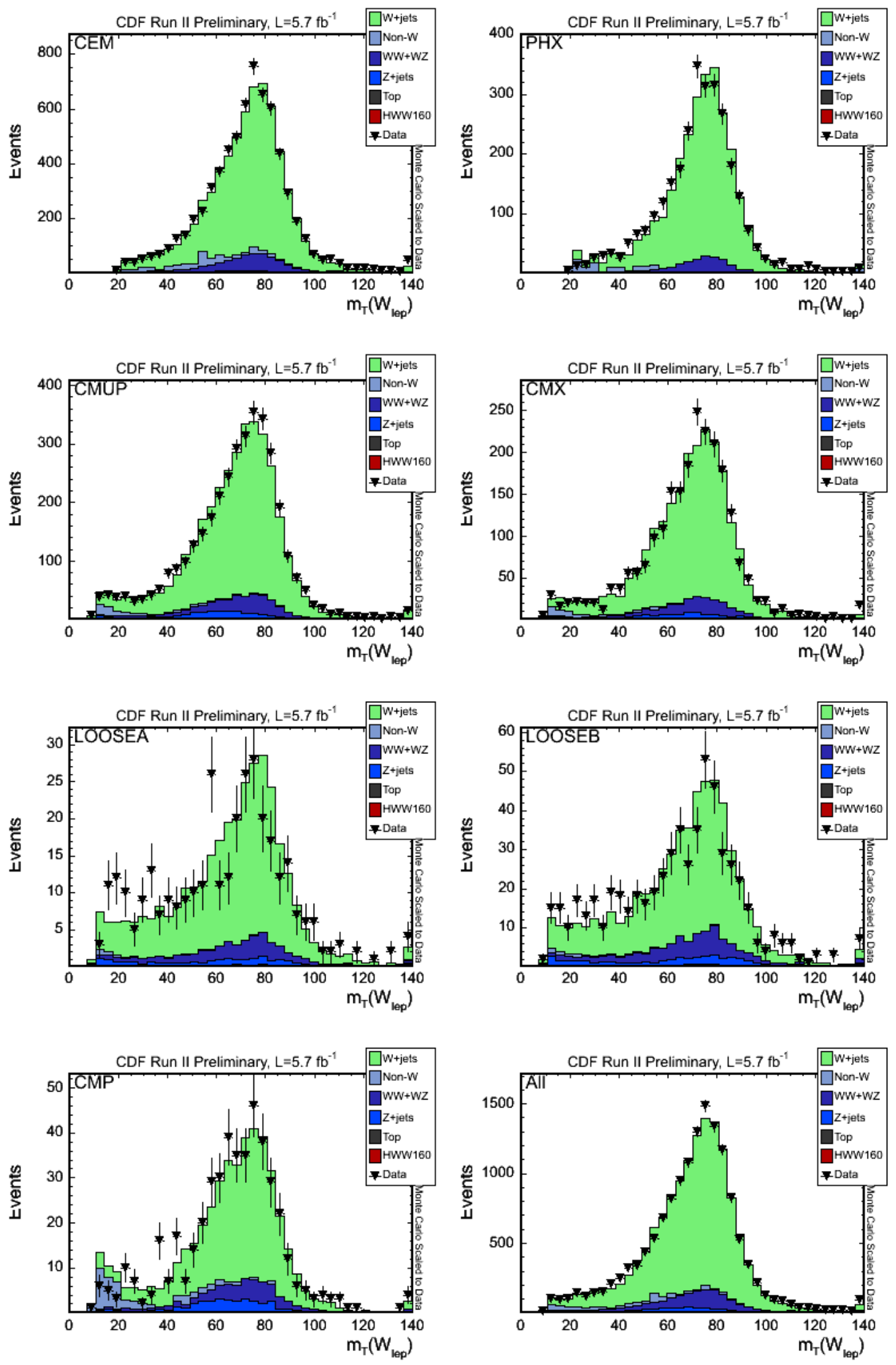

Figure 34: Transverse mass of leptonic $W\left(\boldsymbol{E}_{T}+\right.$ lepton system). 

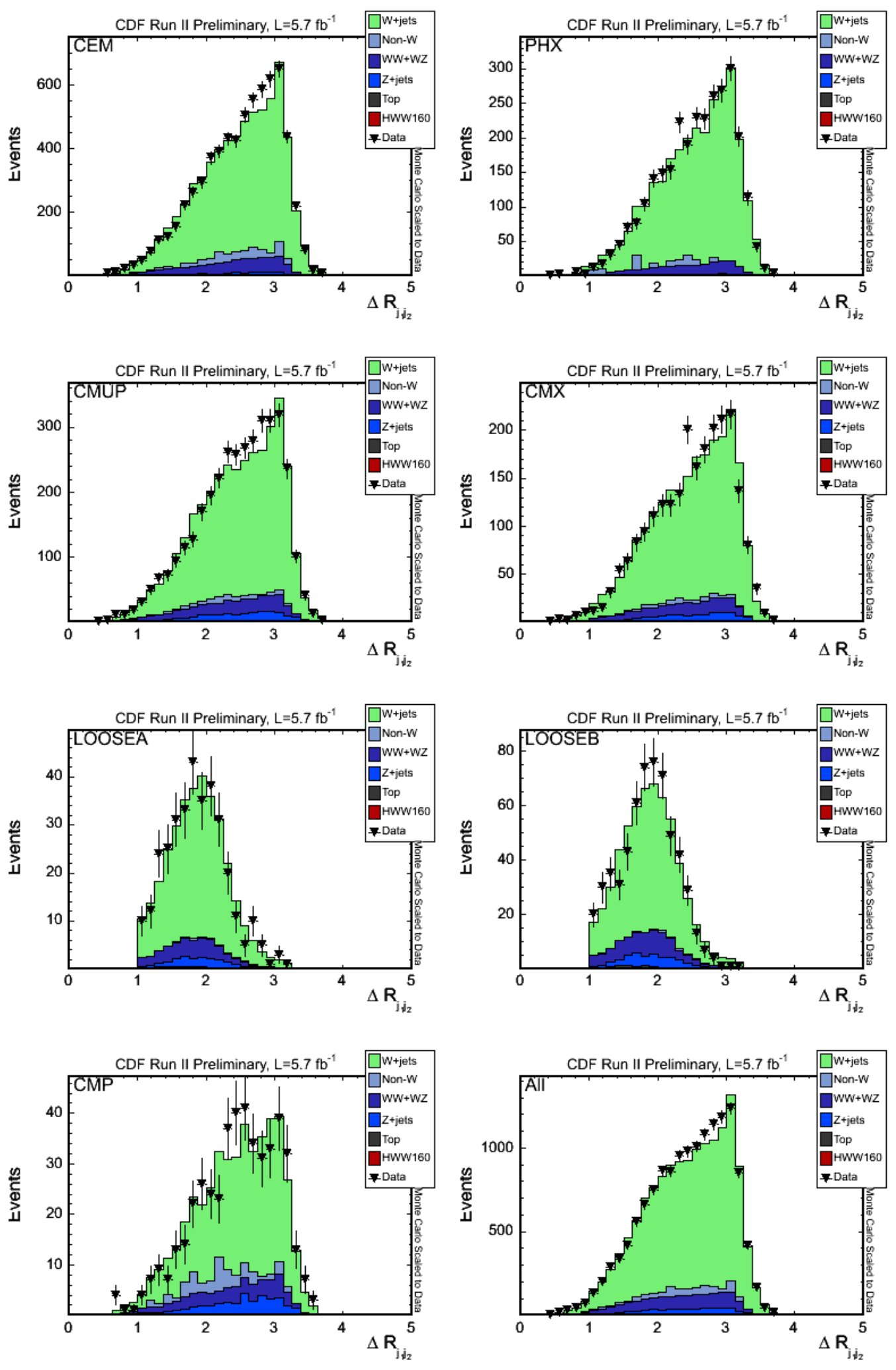

Figure 35: $\Delta R$ between the two jets. 

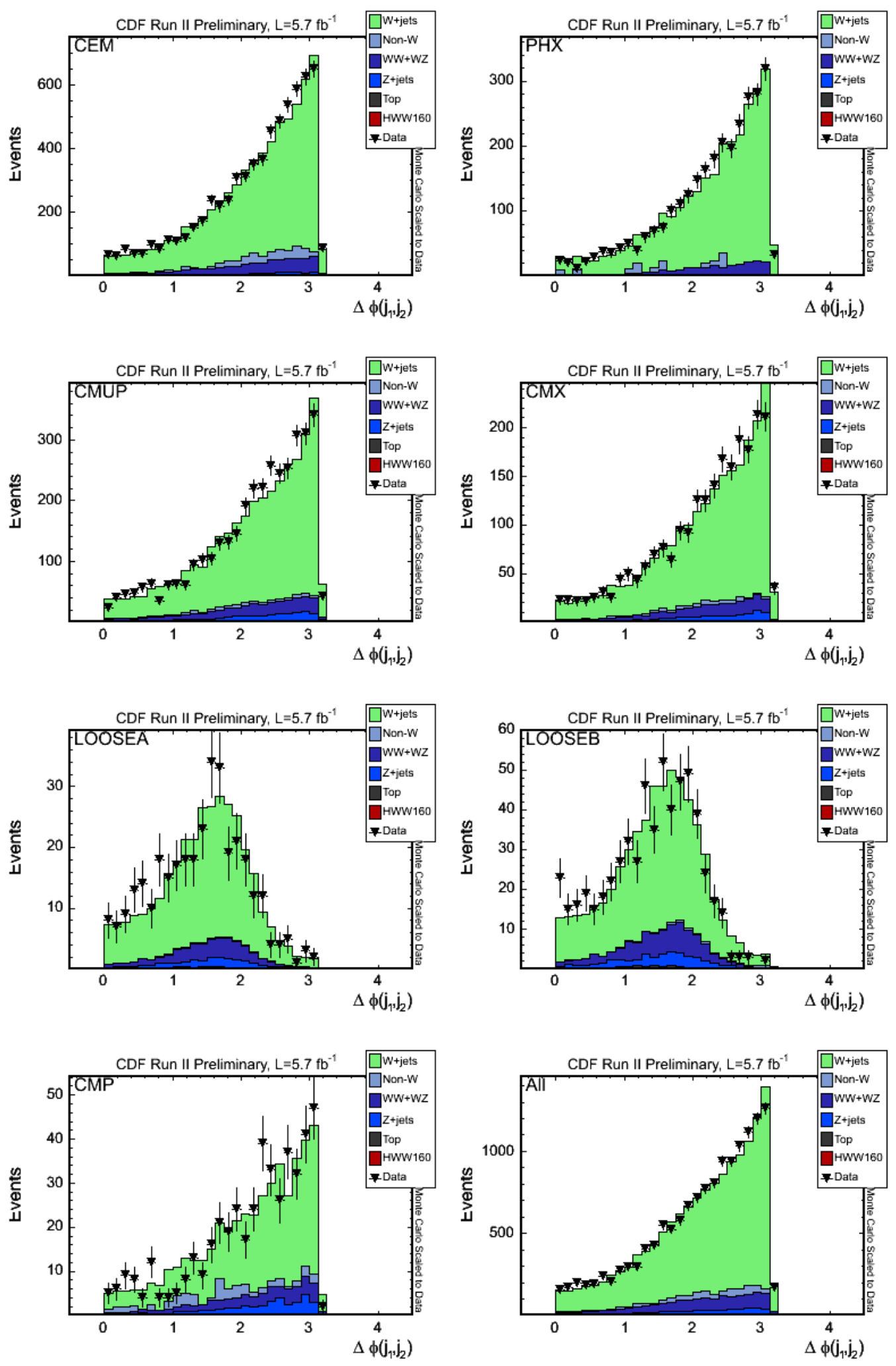

Figure 36: $\Delta \phi$ between the two jets. 

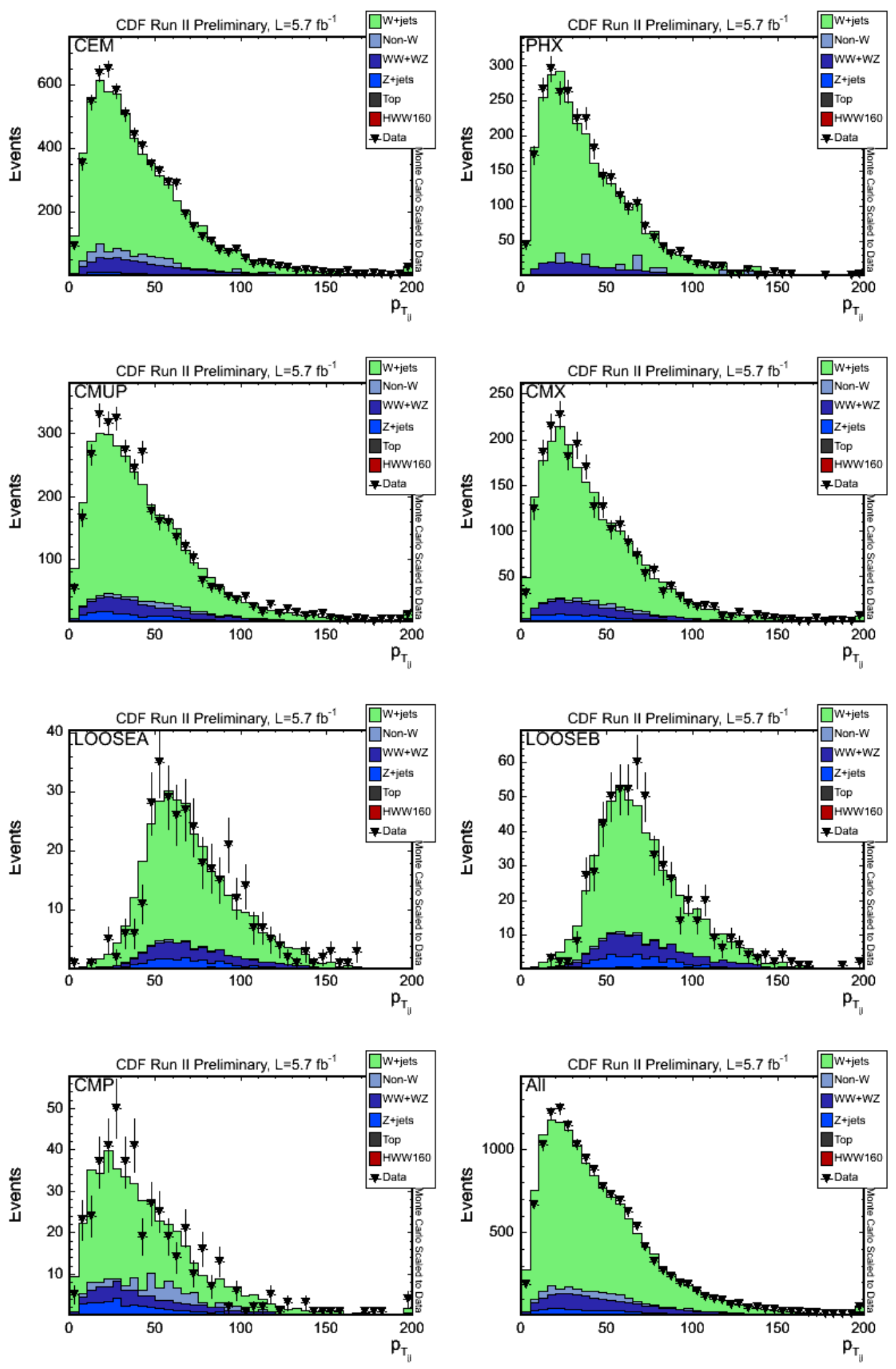

Figure 37: $p_{T}$ of the dijet system. 

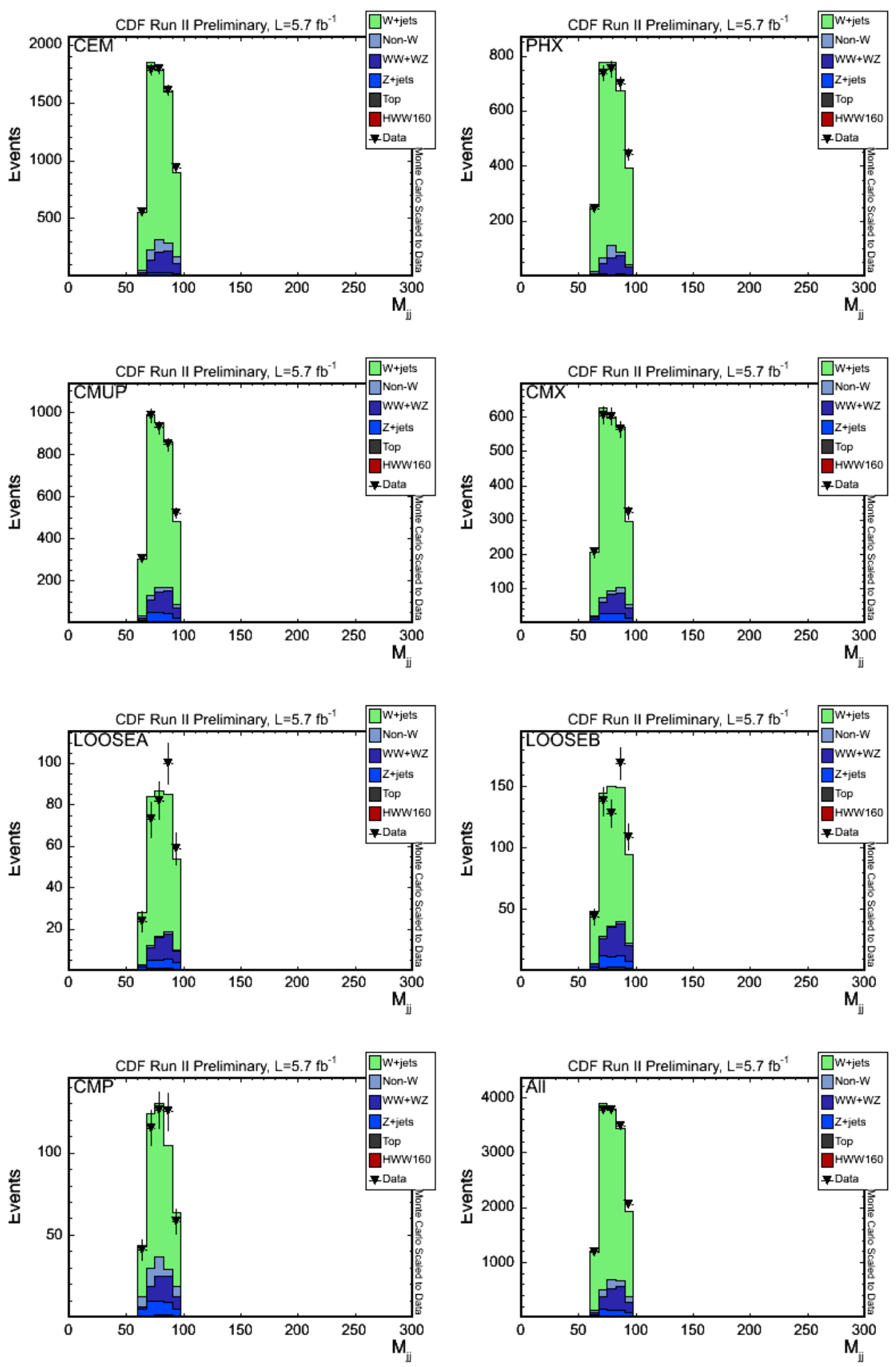

Figure 38: Invariant mass of the dijet system. 


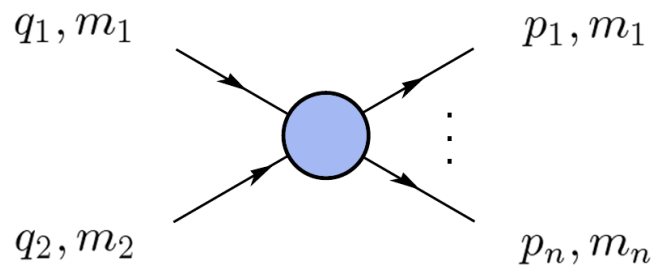

Figure 39: Definition of variables for a process with two particles in the initial state and an $n$ particle final state

\section{Matrix Element Method}

The matrix element method is based on the evaluation of event probability densities for signal and background processes which are obtained through the Standard Model differential cross section calculation. In a second step, a discriminant is defined which has a different shape for signal and background processes. The discriminant and shapes vary depending on the considered Higgs mass.

The different steps are explained in this chapter, starting out with the event probabilities and followed by the transfer function which links observable variables one measures and the production variables. In the next section, the Event Probability Density, which is constructed from the different event probabilities, is presented, followed in turn by an overview of the signal and background processes for which matrix elements are calculated. The next section takes a closer look at the effectiveness of the EPD. The final step of the analysis is then the calculation of the limits which is explained in the last section of this chapter.

\subsection{Event Probabilities}

The cross section for a process with two particles in the initial state (with momenta $q_{1}$ and $q_{2}$ and masses $m_{1}$ and $m_{2}$ ) and $n$ particles in the final state (with momenta $p_{1}, \cdots, p_{n}$ and masses $\left.m_{1}, \cdots, m_{n}\right)$ as illustrated in Fig. 39 is

$$
d \sigma=\frac{(2 \pi)^{4}|\mathcal{M}|^{2}}{4 \sqrt{\left(q_{1} \cdot q_{2}\right)^{2}-m_{1}^{2} m_{2}^{2}}} d \Phi_{n}\left(q_{1}+q_{2} ; p_{1}, \ldots, p_{n}\right)
$$

where $\mathcal{M}$ is the Lorentz invariant matrix element for the interaction and $d \Phi_{n}$ is an element of $n$-body phase space given by

$$
d \Phi_{n}\left(q_{1}+q_{2} ; p_{1}, \ldots, p_{n}\right)=\delta^{4}\left(q_{1}+q_{2}-\sum_{i=1}^{n} p_{i}\right) \prod_{i=1}^{n} \frac{d^{3} p_{i}}{(2 \pi)^{3} 2 E_{i}}
$$

where $E_{i}$ and $p_{i}$ are the energies and momenta of the particles in the final state. By normalizing the differential cross-section to the total cross-section,

$$
P_{\text {event }} \sim \frac{d \sigma}{\sigma}
$$


one can define a probability density for the corresponding process. If all four momenta of the particles in the initial and final state were known, one could use equation (9) to find the event probability. However, several effects need to be considered:

1. The initial state interaction is initiated by partons inside the protons and antiprotons.

2. The final state parton measurements are affected by the experimental resolution which is non-zero and should not be ignored

3. Furthermore, the neutrinos in the final state are not identified directly

The first complication is remedied by folding the differential cross section over the parton distribution functions, which give the probability density of finding a certain parton with a given longitudinal momentum fraction $x$ at momentum transfer $Q^{2}$ of the collision. The parton distribution functions (PDFs) are evaluated according to the CTEQ6.1 parametrization [13]. In order to account for the last two complications, one can integrate over the particle momenta that are either unmeasured (e.g. $p_{z}$ of the neutrino) or not measured well (e.g. due to resolution effects). The integration is carried out as one would like to sum over all possible particle variables $y$ leading to the observed set of variables $x$ measured by the detector. The mapping between the possible particle variables and the measured variables is established with the transfer function $W(y, x)$.

When incorporating these adaptations, one obtains

$$
P_{\text {event }}(x)=\frac{1}{\sigma} \int d \sigma(y) d q_{1} d q_{2} f\left(y_{1}\right) f\left(y_{2}\right) W(y, x)
$$

where $d \sigma(y)$ is the differential cross section in terms of the particle variables $y$ and $f\left(y_{i}\right)$ are the PDFs. Furthermore, one neglects masses and transverse momenta of the initial state partons so that

$$
\sqrt{\left(q_{1} \cdot q_{2}\right)^{2}-m_{q_{1}}^{2} m_{q_{2}}^{2}} \simeq 2 E_{q_{1}} E_{q_{2}} \quad \text { for } \quad q_{i}^{2} \simeq 0, q_{i, T} \simeq 0
$$

When taking all the previously mentioned modifications into account, the event probability becomes

$$
P_{\text {event }}(x)=\frac{1}{\sigma} \int 2 \pi^{4}|\mathcal{M}|^{2} \frac{f\left(y_{1}\right)}{\left|E_{q_{1}}\right|} \frac{f\left(y_{2}\right)}{\left|E_{q_{2}}\right|} W(y, x) d \Phi_{4} d E_{q_{1}} d E_{q_{2}}
$$

In the analysis, the event probability densities were calculated for the $H \rightarrow W W$ signal as well as for the s-channel and t-channel single top, $t \bar{t}, W b \bar{b}, W c \bar{c}, W c j$, mistags $(W g g)$ and diboson background processes. The matrix element $\left(|\mathcal{M}|^{2}\right)$ for the event probability density is calculated at leading order by using the HELAS (HELicity Amplitude Subroutines for Feynman Diagram Evaluations) package; the subroutines are generated by the MadGraph program. Different subroutines are employed for calculating event probabilities for the $H \rightarrow W W$ signal and $W W, W Z$, single top, $W g g, W g j, W c c$, $W c j, W b b$ and $t \bar{t}$ background hypotheses. The transfer function is normalized such that $\int W(y, x) d x=1$ which expresses the assumption that a set of partonic variables $y$ will 
always turn into a set of detector variables $x$. Consequently, integrating over all $x$ will lead to unity,

$$
\frac{1}{\sigma} \int P_{\text {event }}(x) d x=\frac{1}{\sigma} \int d \sigma(y) d x d q_{1} d q_{2} f\left(y_{1}\right) f\left(y_{2}\right) W(y, x)=1
$$

\subsection{Transfer Function}

The link between the observable variables $x$ one measures and the production variables $y$ is established by the transfer function (or "detector response"), which provides the probability of measuring a set of observables $x$ corresponding to the set of production variables $y$. One distinguishes different cases: In the case of well-measured objects, the transfer function can be taken as a $\delta$ function so that the measured momenta are assumed to be the final state momenta. In the other extreme case of unmeasured quantities, the transfer function is taken as unity as is the case for neutrinos (but not their transverse momentum as it can be inferred from energy and momentum conservation). When the detector resolution cannot be ignored, the transfer function is taken as a Gaussian-type function.

\subsection{Event Probability Discriminant}

The background processes dominate by orders of magnitude, requiring a discriminant. The probabilities for all processes are thus combined to form one discriminant to specify each event; this discriminant has a different shape for background-like events than for signal-like events. The event probability is used to form an event probability discriminant (EPD), which is a distribution that separates signal from background and which can be fit to the data. The choice of the combination of the event probabilities to give the EPD is arbitrary to a certain degree, but the most intuitive discriminant is the ratio of signal over signal + background probability,

$$
E P D=\frac{P_{s}}{P_{s}+P_{b g}}
$$

which is closer to zero for more background-like events and closer to unity for signal-like events. The probability $P_{s}$ is the probability of the signal process and $P_{b g}$ is the sum of all background processes. The previously introduced $P_{i}$ are not true probabilities as they are not normalized; the normalization is done by calculating them for each event in large Monte Carlo samples, finding the maximal $P_{i}$ over all $\mathrm{MC}$ events and then normalizing the original $P_{i}$ to it. In the present analysis, the background event probability is given by

$$
P_{b g}=P_{W W}+P_{W Z}+P_{\text {single top }}+P_{W g g}+P_{W g j}+P_{W c c}+P_{W b b}+P_{W c j}
$$

so that

$$
E P D=\frac{P_{H W W}}{P_{H W W}+\left(P_{W W}+P_{W Z}+P_{\text {single top }}+P_{W g g}+P_{W g j}+P_{W c c}+P_{W b b}+P_{W c j}\right)}
$$

One obtains characteristic shapes for background and signal processes as illustrated in Fig. 40. In order to make the signal shape more different from the background 

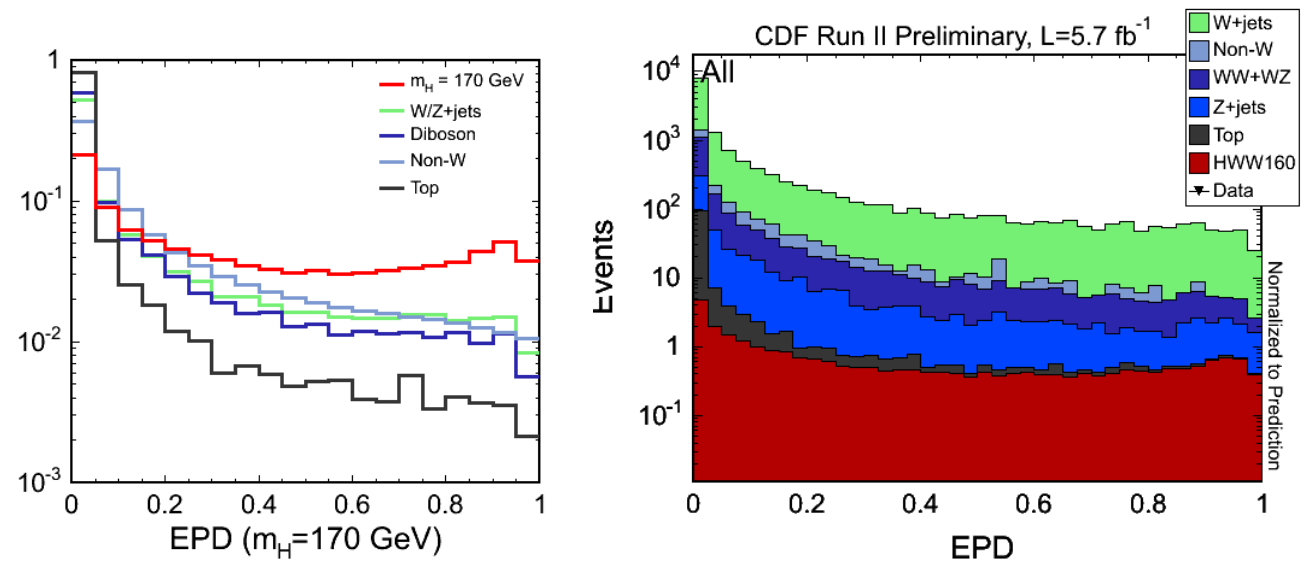

Figure 40: Comparison of the different shapes in a Monte Carlo sample for a Higgs mass of $m_{H}=170 \mathrm{GeV}$. The x-axis gives the event probability discriminant value whereas the $\mathrm{y}$ axis represents the relative number of events at the corresponding EPD value (normalized to unit area for each contribution); the stacked plot on the right includes the expected normalizations.

shapes, one introduces coefficients for the event probabilities in the definition of the event probability discriminant:

$$
E P D=\frac{c_{s} P_{s}}{c_{s} P_{s}+\tilde{P}_{b g}}
$$

where

$$
\begin{aligned}
\tilde{P}_{b g}= & c_{W W} P_{W W}+c_{W Z} P_{W Z}+c_{\text {single top }} P_{\text {single top }}+ \\
& c_{W g g} P_{W g g}+c_{W g q} P_{W g q}+c_{W c c} P_{W c c}+c_{W b b} P_{W b b}+c_{w c j} P_{W c j}
\end{aligned}
$$

The coefficients $c_{i}$ change the relative weight of the different contributions and with it the shape of the EPD; the coefficients have been optimized in this analysis to yield the best figure of merit with the cuts described in Chapter 4 . The optimization is carried out using Monte Carlo models only, no data, and the expected yields of the signal and backgrounds processes.

\subsection{Signal and Background Processes}

Matrix Element probabilities are evaluated for the signal process (at all masses) and a selection of background processes listed on the following pages. The leading order diagrams used in the matrix element calculation are provided by MADGRAPH [20].

The $W+$ jets contributions are split up into different contributions: $W+$ two gluons $(W g g), W+$ one gluon and one quark $(W g q), W+$ two $b$ quarks $(W b b)$, and $W+$ one $c$-quark and one gluon $(W c g)$. Some of the corresponding diagrams are given in Fig. 41 for $W g g$ and Fig. 42 for $W g j$. 

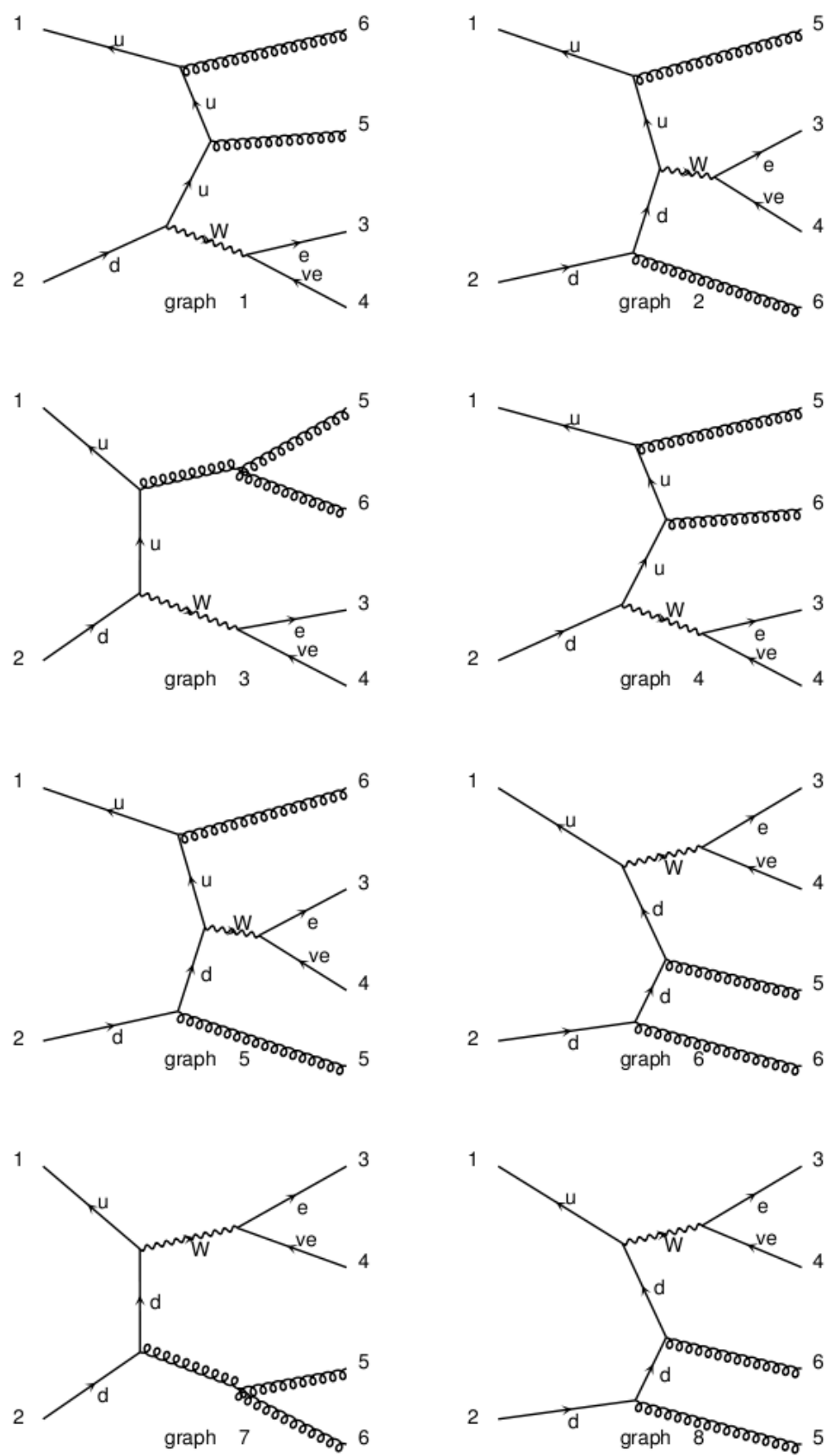

Figure 41: Examples of $W g g$ production diagrams 

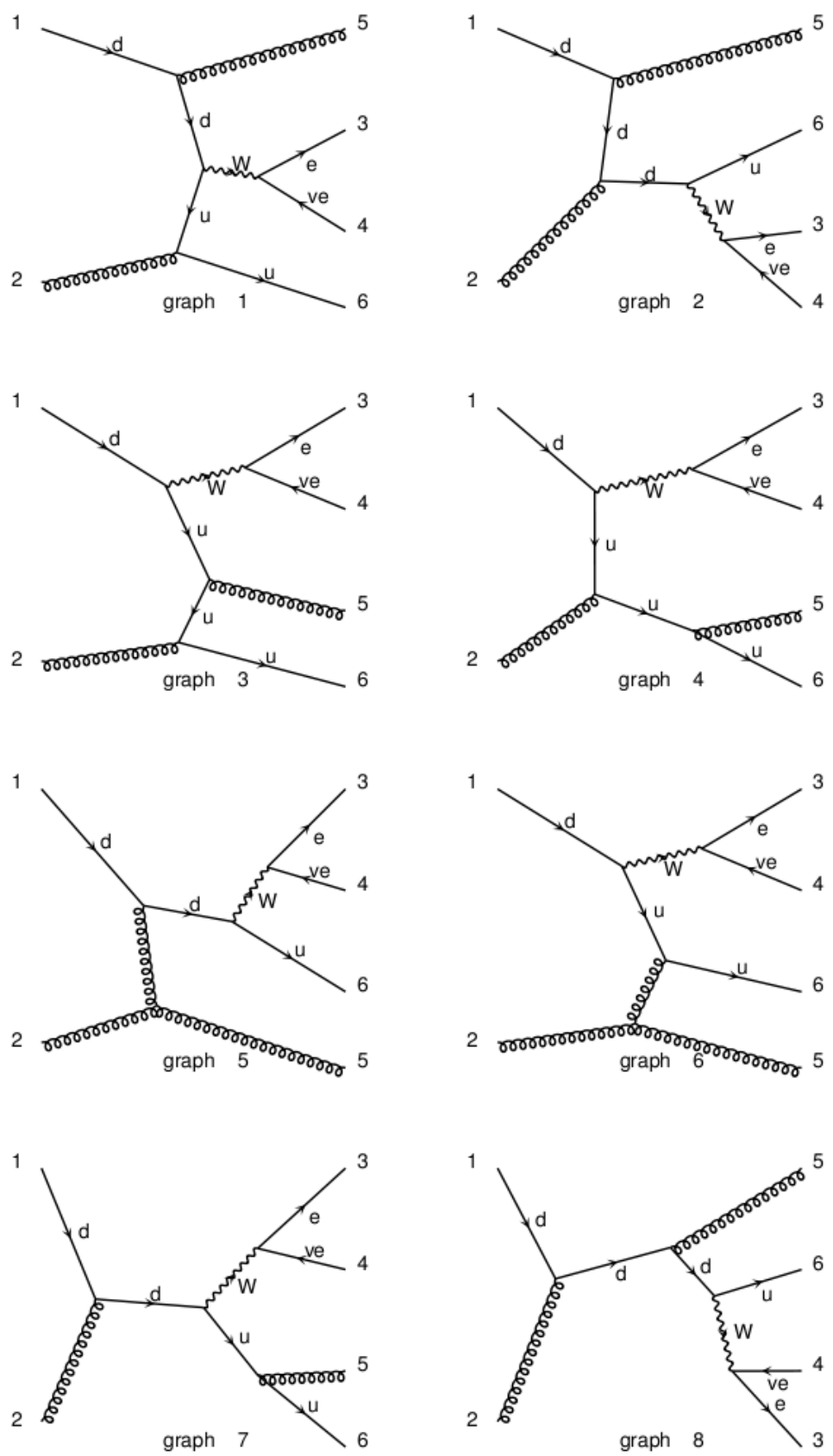

Figure 42: Examples of $W g q$ production diagrams 


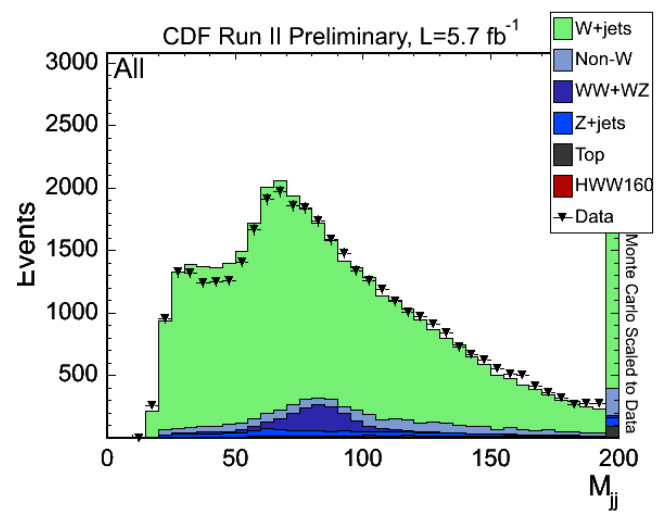

(a) $M_{j j}$ distribution for $0<E P D<0.25$

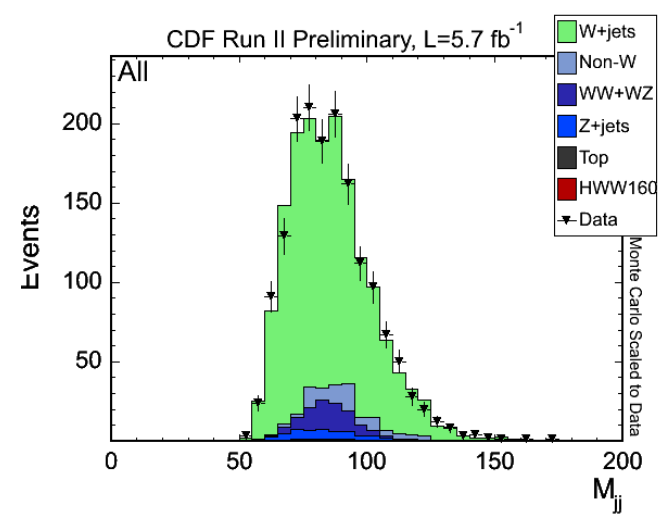

(c) $M_{j j}$ distribution for $0.5<E P D<0.75$

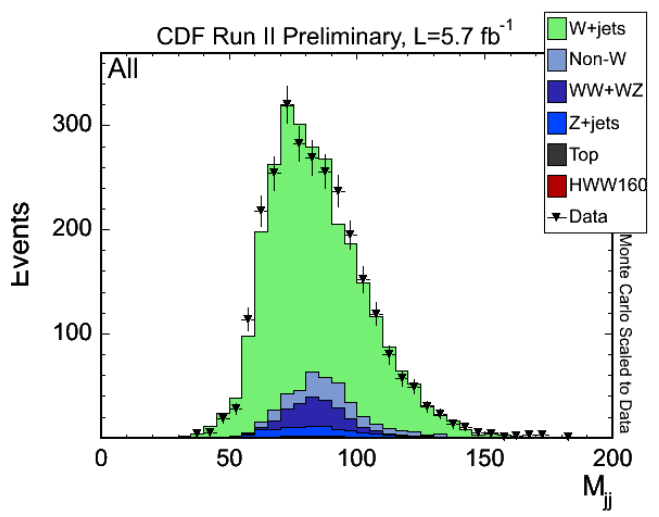

(b) $M_{j j}$ distribution for $0.25<E P D<0.5$

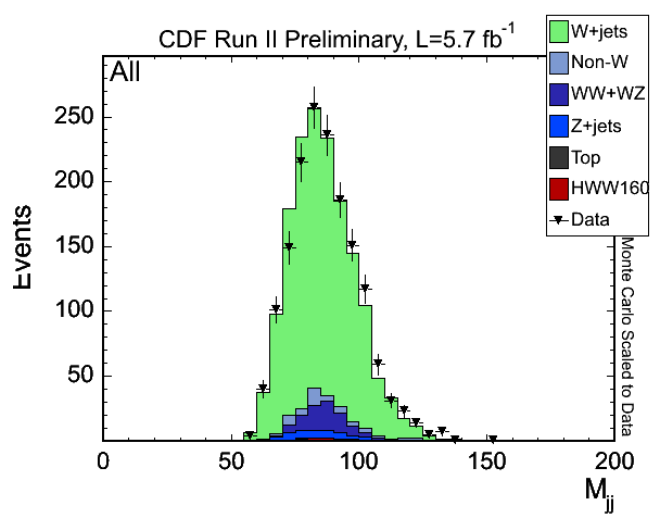

(d) $M_{j j}$ distribution for $0.75<E P D<1$

Figure 43: Distribution of the dijet mass $M_{j j}$ in four EPD bins. As the EPD approaches unity, the peak near the $W$ mass becomes more clearly visible.

Additional matrix elements include those for single top (s-channel and two t-channels). For $t \bar{t}, Z+$ jets, and QCD multi-jet backgrounds, additional assumptions are necessary and no matrix element calculation is carried out.

\subsection{Effectiveness of the Event Probability Discriminant}

The effectiveness of the EPD can be seen when plotting the invariant dijet mass, which should peak at around the $W$ mass for signal-like events. For this test, the dijet mass cut (described in Section 4.1.7) was lifted. The corresponding stack plots for the four $\mathrm{EPD}$ regions $(\mathrm{EPD}<0.25,0.25<\mathrm{EPD}<0.5,0.5<\mathrm{EPD}<0.75$ and $0.75<\mathrm{EPD})$ are given in Fig. 43 . While the top left plot in Fig. 43 contains the full range, the other three plots increasingly clearly show a peak in the $W$ mass range. Furthermore, the signal to background ratio increases as the EPD reaches unity. In the case of $m_{H}=160 \mathrm{GeV}$, the signal to background ratio is on the order of 1:1000 over the full EPD range whereas it is about 1:60 in the highest EPD region defined as $0.975 \leq \mathrm{EPD} \leq 1$ region (corresponding to the highest EPD bin in the templates). 


\subsection{The Likelihood Fit}

The limits are calculated by fitting the shape of the Event Probability Discriminant (EPD) in the data to the sum of background and signal shapes. The details of the fitting procedure are explained in this subsection.

\subsubsection{Fitting Procedure}

In this analysis, a Bayesian approach to a maximum likelihood fit is chosen. Given a set of observables $x$ based on which we determine a quantity $y$, the posterior probability density function $p(y \mid x)$ can be determined using Bayes' theorem:

$$
p(y \mid x)=\frac{\mathcal{L}(x \mid y) \pi(y)}{\int \mathcal{L}\left(x \mid y^{\prime}\right) \pi\left(y^{\prime}\right) d y^{\prime}}
$$

where $\mathcal{L}$ is the likelihood function and $\pi(y)$ is the prior probability distribution function for $y$. [28]. The fitted limit corresponds to the value at which the probability density function is maximal. The one $\sigma$ bands are determined by finding the smallest interval around this value that covers $68 \%$ of the total area of the probability density function.

The MCLIMIT package, which relies on Markov chains, is used for the limit calculation. [29][30].

\subsubsection{Treatment of Systematic Uncertainties}

Systematic normalization and shape uncertainties are incorporated into the likelihood as nuisance parameters, conforming with a fully Bayesian treatment [25]. The uncertainties are marginalized or integrated over to derive a likelihood that is only a function of the parameters of interest. In the present analysis, we want to establish a limit on the $H$ production cross section times the branching ratio of the Higgs boson to decay to two $W$ in units of the SM, so we parametrize $\beta=\sigma^{f i t} / \sigma_{S M}$. Bayesian measurements require a prior which represents the experimenter's belief in the distribution of values of the outcome before examining the result. In this analysis, a flat prior is used for all nonnegative values of $\beta$. Gaussian priors, which are normalized by their estimated standard deviations to obtain standard normal distributions, are used for all nuisance parameters.

When integrating over the prior p.d.f. of a nuisance parameter, some region may contribute more to the likelihood than others and if some regions of the p.d.f. lead to very low likelihoods, they can effectively be excluded. In such a case, the likelihood fit is constraining the value of a nuisance parameter, and by examining the posterior p.d.f. of that nuisance parameter one can conclude the regions strongly favored by the fit.

If there are many nuisance parameters, integrating over all of their prior p.d.f. is a standard approach, but can run into difficulty when a nuisance parameter is strongly constrained and many integration points fall outside of the region relevant to the fit. Since that is a concern in our fit, we use a Markov Chain Monte Carlo integration, which more efficiently finds the regions of nuisance parameter space contributing most to the likelihood [31] 


\subsubsection{The Likelihood Function}

The definition of the likelihood function starts from the standard likelihood for a Poison distributed variable. In a simple counting experiment without systematic uncertainties, and with only one signal process and one background process, the likelihood is simply given by

$$
L(\beta)=\frac{\mu^{n}}{n !} e^{-\mu}
$$

where $\mu$ is the number of expected events given $\beta$ (with $N_{b}$ background events and $N_{s}$ signal events, $\mu=\beta N_{s}+N_{b}$ ), $N_{s}$ is the number of signal events expected from the Standard Model prediction, $N_{b}$ is the number of background events, $n$ is the number of observed data events, and $\beta$ is the fraction of the standard Model expectation as defined above. When we consider a histogram instead of a simple counting experiment, we can generalize 113 as we are dealing with a combination of many statistically independent counting experiments, so we obtain

$$
L(\beta)=\prod_{k=1}^{n_{b i n s}} \frac{\mu_{k}^{n_{k}}}{n_{k} !} e^{-\mu_{k}}
$$

with $\mu_{k}=\beta N_{s, k}+N_{b, k}$, and $k$ being the index of each bin. We can adapt the notation to reflect the change from one background process to a number of background processes, so we write

$$
\mu_{k}=\beta N_{s, k}+\sum_{j=1}^{n_{b}} N_{j, k}
$$

with $j$ running over background processes only. The first term thus represents the signal and the second term is the sum over all background processes, with $n_{b}$ being the number of background processes. If there are several channels, the bin index $k$ can be defined to include the bins of all histograms.

So far, systematic uncertainties have not been considered. The simplest case to incorporate systematic uncertainties involves uncertainties on the background estimate that are symmetrical; they are treated as nuisance parameters, i.e. numbers whose values affect the result but are not themselves of interest to the analysis. Each systematic uncertainty $i$ (up to the total number of systematic uncertainties $n_{\text {sys }}$ ) is assigned a nuisance parameter $\delta_{i}$. The $\pm 1 \sigma$ change in the normalization of the process $j$ due to systematic uncertainty $i$ is given by $\epsilon_{i j}$. Each nuisance parameter is assigned a prior which reflects the degree of belief of different possible values and is then marginalized, or integrated, to calculate the reduced likelihood as a function only of the parameter of interest:

$$
L(\beta)=\int_{-\infty}^{\infty} \prod_{i=1}^{n_{\text {sys }}} d \delta_{i} \frac{1}{\sqrt{2 \pi}} \exp \left(-\frac{\delta_{i}^{2}}{2}\right) \prod_{k=1}^{n_{\text {bin }}} \frac{\mu_{k}^{n_{k}}}{n_{k} !} e^{-\mu_{k}}
$$

and

$$
\mu_{k}=\beta N_{s, k}+\sum_{j=1}^{n_{b}} N_{j, k} S_{j}
$$


where $S_{j}$ is a systematic factor given by

$$
S_{j}=\prod_{i=1}^{n_{\text {sys }}}\left(1+\delta_{i} \epsilon_{i j}\right)
$$

The systematic factor described above takes into account the change in the normalization or rate of a given process due to a systematic uncertainty. In addition, the contribution of the systematics may vary with the histogram bin (i.e. the systematic uncertainty may affect the shape of the histogram). To take this into account, we define $\kappa_{j i k}$ as the change in the value of bin $k$ in process $j$ due to uncertainty $i$. The systematic factor is then bin-dependent, meaning

$$
\mu_{k}=\beta N_{s, k} S_{s, k}+\sum_{j=1}^{n_{b}} N_{j, k} S_{j, k}
$$

and

$$
S_{j, k}=\prod_{i=1}^{n_{s y s}}\left(1+\delta_{i} \epsilon_{j i}\right)\left(1+\delta_{i} \kappa_{j i k}\right) .
$$

The shifts caused by systematic effect can be asymmetrical about the mean of a certain parameter. In that case, the single Gaussian prior p.d.f. for the nuisance parameter is replaced by two Gaussians connected by a Heaviside step function at zero. The resulting distribution is discontinuous, yet its effect on the output is continuous since the effect of the systematic vanishes at zero. This prior gives equal probability to positive and negative values of the uncertainty. We can thus split up $\epsilon_{j i}$ (and $\kappa_{j i k}$ ) into $\epsilon_{j i+}$ and $\epsilon_{j i-}$ $\left(\kappa_{j i k+}\right.$ and $\left.\kappa_{j i k-}\right)$, so that the systematic factor becomes

$$
S_{j, k}=\prod_{i=1}^{n_{s y s}}\left(1+\left|\delta_{i}\right|\left(\epsilon_{j i+} H\left(\delta_{i}\right)+\epsilon_{j i-} H\left(-\delta_{i}\right)\right)\right)\left(1+\left|\delta_{i}\right|\left(\kappa_{j i k+} H\left(\delta_{i}\right)+\kappa_{j i k-} H\left(\delta_{i}\right)\right)\right) .
$$

The $\delta_{i}$ correlate shape and normalization uncertainties due to the same source.

The full likelihood is thus given by equation 16, where $\mu_{k}$ is defined in equation 19 and the systematic factor is defined in equation 21 . 


\section{Systematic Uncertainties}

Systematic uncertainties can bias the outcome of the analysis, since the matrix element method relies on the description of data by MC modeling. Their impact manifests itself in various ways, as they can change the event yield (normalization or rate) as well as the shape of the discriminant distribution for signal and background; they thus need to be incorporated into the analysis. Several systematic uncertainties from different sources are included: (1) background normalization ${ }^{4}$ (2) signal normalization, (3) jet energy scale, (4) parton distribution functions, (5) initial state and final state radiation (ISR/FSR), (6) factorization and renormalization scale $\left(Q^{2}\right)$ in Alpgen, and (7) event selection efficiency and luminosity. These uncertainties are explained further in the ensuing sections, an overview is presented in Table 7.

\begin{tabular}{|l|l|c|c|c|}
\hline \hline Process & Source & Rate $-1 \sigma$ & Rate $+1 \sigma$ & Shape? \\
\hline$H W W$ & Normalization & $-10 \%$ & $10 \%$ & no \\
$W W, W Z, Z Z$ & Normalization & $-10 \%$ & $10 \%$ & no \\
$W+$ jets & Normalization & $-20 \%$ & $20 \%$ & no \\
$Z+$ jets & Normalization & $-15 \%$ & $15 \%$ & no \\
$t \bar{t}$ and single top & Normalization & $-12 \%$ & $12 \%$ & no \\
non- $W$ & Normalization & $-40 \%$ & $40 \%$ & no \\
\hline$H W W$ & JES & $-7 \%$ & $5 \%$ & yes \\
\hline$H W W$ & PDF & $-2 \%$ & $2 \%$ & no \\
$H W W$ & IFSR & $-6 \%$ & $6 \%$ & no \\
\hline$W+$ jets & $Q^{2}$ & $0 \%$ & $0 \%$ & yes \\
\hline \hline All MC & Event selection efficiency & $-2 \%$ & $2 \%$ & no \\
All MC & Luminosity & $-6 \%$ & $6 \%$ & no \\
\hline \hline
\end{tabular}

Table 7: Summary of systematic uncertainties. All MC refers to all processes except non- $W$ and $W+$ jets, whose normalizations are determined by fit to the data.

\subsection{Background Normalization}

The first uncertainties considered are the uncertainties on the background normalization, which are taken from the estimates previously described in Section 5 . The background normalization uncertainties reflect the uncertainty on the cross sections in the case of $Z+$ jets, $t \bar{t}$, and single top backgrounds. An overview is given in Table 7 . In the fit, the normalization of the single top and $t \bar{t}$ backgrounds are correlated.

Furthermore, a conservative $40 \%$ uncertainty is applied to the non- $W$ background normalization and a $20 \%$ uncertainty is assigned to the $W+$ jets normalization, which is much higher than either the measured or theoretical uncertainty of the cross section. However, when carrying out the likelihood fit, the $W+$ jets normalization is constrained

\footnotetext{
${ }^{4}$ The background normalization uncertainties are considered part of the statistical uncertainty but are described in detail at this point nonetheless
} 
to better than $2 \%$; the $20 \%$ uncertainty is thus not a real constraint (the $W+$ jets normalization is a free parameter in the fit).

\subsection{Signal Normalization}

An uncertainty of $10 \%$ is used for the signal normalization.

\subsection{Jet Energy Scale}

In order to evaluate the impact of the uncertainty on the jet energy scale, the analysis is rerun with the energy scale being scaled by $\pm 1 \sigma$. The effect of the uncertainty is investigated for the signal templates only, as previous analyses have shown the impact on the background templates to be minimal. [17].

The signal acceptance changes by $+5 \% /-7 \%$ (for $m_{H}=160 \mathrm{GeV}$ ) as a result of the jet energy uncertainty. The change in signal acceptance depends on the assumed Higgs mass, but we use the largest number for all Higgs masses. Both the matrix elements and the EPD are re-calculated and shape uncertainties are assigned both for the signal process and the $W+$ jets background. Examples of the change in the templates are given in Fig. 44, where the top plots illustrate the central shape and the bottom plots show the ratio of the up-corrected template to the central one (green), and the ratio of the down-corrected template to the central one (blue). The plotted shapes are the sum over all considered lepton categories. The shape uncertainties are smoothed internally over 5 bins in the likelihood fit.

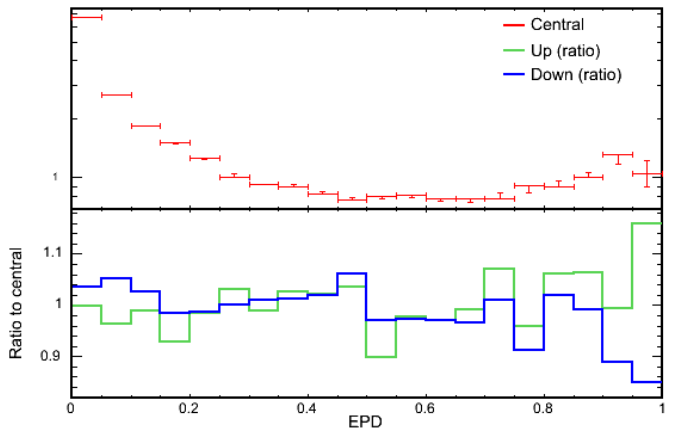

(a) Change in the EPD templates for $m_{H}=160 \mathrm{GeV}$

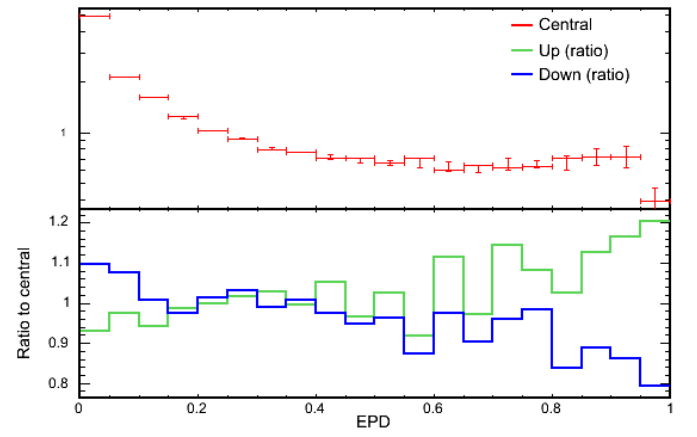

(b) Change in the EPD templates for $m_{H}=180 \mathrm{GeV}$

Figure 44: Changes in the EPD templates associated with the JES uncertainty for Higgs masses of (a) $m_{H}=160 \mathrm{GeV}$ and (b) $m_{H}=180 \mathrm{GeV}$, along with ratios of upcorrected to central template (green) and down-corrected to central template (blue). The uncertainty on the jet energy scale has a large effect on the shape of the templates. 


\subsection{PDF}

A $2 \%$ uncertainty is applied to the signal, based on CDF note 9923 [21]. The PDF uncertainty for the signal has not been explicitly calculated.

\subsection{Initial State Radiation / Final State Radiation}

A $6 \%$ acceptance uncertainty is applied, based on the change due to initial state radiation/final state radiation. The uncertainty for the signal was not explicitly evaluated.

\subsection{Factorization and Renormalization Scale}

Another systematic uncertainty that needs to be included is the so-called $Q^{2}$ uncertainty which is related to the renormalization scale. The ALPGEN event generator, which is used for $W+$ jets events, uses the renormalization and factorization scale to solve divergences arising due to gluon splitting and is thus susceptible to the renormalization scale, since it carries out leading order matrix element calculations. The default choice is $Q^{2}=m_{W}^{2}+\sum m_{T}^{2}$, where $m_{W}$ represents the mass of the $\mathrm{W}$ boson and $\sum m_{T}$ is the sum over the transverse masses of the final state partons. However, since these values are not known (and not physically accessible), one assigns an uncertainty. In order to extract the impact of the uncertainty, the $W+$ jets samples are thus generated once with the default scale doubled and once halved. The change does not only affect the shape of the EPD (as illustrated in Fig. 45) but also the shape of several distributions, some of which are shown in Figure 46. The change in the EPD shape is considered a two $\sigma$ uncertainty and it is not truncated.

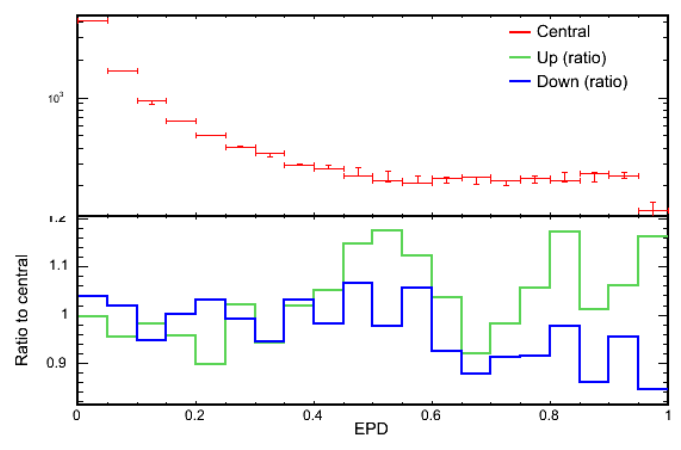

(a) Change in templates for $m_{H}=150 \mathrm{GeV}$

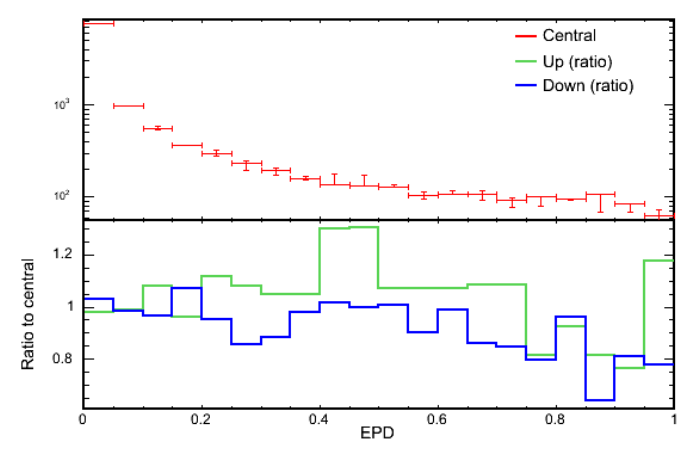

(b) Change in templates for $m_{H}=160 \mathrm{GeV}$

Figure 45: Change in the $W+$ jets EPD template associated with the $Q^{2}$ uncertainty for an assumed mass of $m_{H}=150 \mathrm{GeV}$ (a) and $m_{H}=160 \mathrm{GeV}$ (b)

\subsection{Event Selection Efficiency and Luminosity}

An uncertainty of $6 \%$ on the luminosity is applied to all processes whose normalization is determined by theory and Monte Carlo $(H \rightarrow W W, W W, W Z, Z Z$, single top, $t \bar{t}$, and $Z+$ jets $)$. Furthermore, a $2 \%$ uncertainty is applied due to trigger and offline lepton 

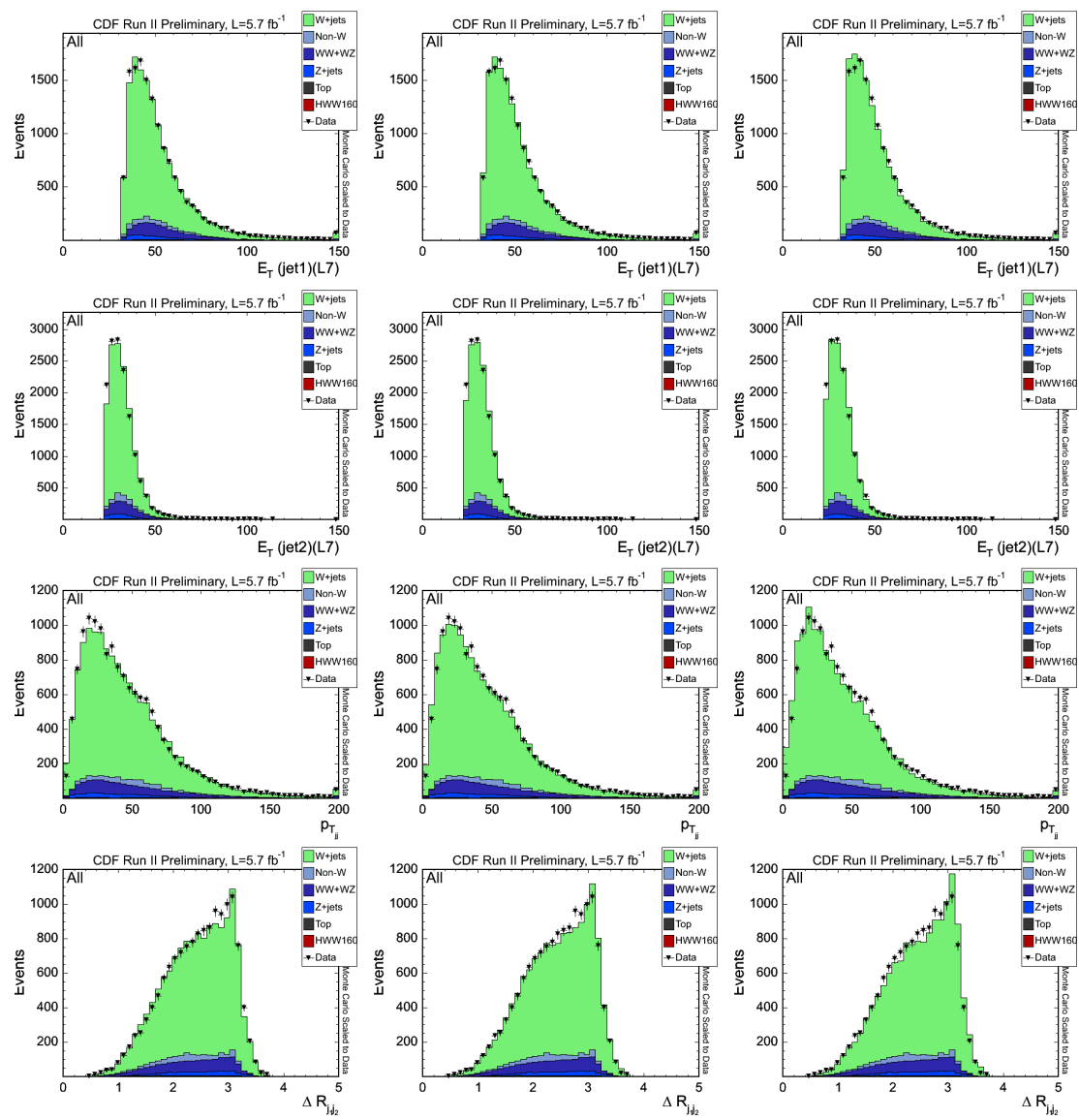

Figure 46: Modeling of various variables with the $Q^{2}$ scale decreased by a factor of $\frac{1}{2}$ (left column), the central choice of $Q^{2}$ (center) and the $Q^{2}$ scale increased by a factor of two (right column). The top row shows the plots for the transverse energy of the harder jet, the second row the transverse energy of the second jet, the third the momentum of the dijet system, and the fourth row gives the plots for the $\Delta \mathrm{R}$ between the two jets.

identification efficiencies.

The effect of the several uncertainties $\left(Q^{2}\right.$ uncertainty, jet energy scale uncertainty and $W+$ jets normalization uncertainty) is analyzed in the results, in Section 8.1 . 


\section{Results}

The analysis is applied to a data sample corresponding to $5.7 \mathrm{fb}^{-1}$ of CDF II data. We compare the EPD output distribution for Higgs masses of 150, 160, 170, 180, 190 and $200 \mathrm{GeV}$ of our candidate events with the sum of predicted $H \rightarrow W W$ signal and background distributions (as shown in Fig. 47 for the case of $m_{H}=160 \mathrm{GeV}$ ).

In order to extract the most probable $H \rightarrow W W$ signal content in the data, we perform the maximum likelihood method described previously in Section 6.6. We perform marginalization with all systematic uncertainties included in the likelihood function. The posterior p.d.f. is obtained using Bayes' theorem:

$$
p\left(\beta_{1} \mid \text { data }\right)=\frac{\mathcal{L}^{*}\left(\text { data } \mid \beta_{1}\right) \pi\left(\beta_{H W W}\right)}{\int \mathcal{L}^{*}\left(\text { data } \mid \beta_{H W W}^{\prime}\right) \pi\left(\beta_{H W W}^{\prime}\right) d \beta_{H W W}^{\prime}}
$$

where $\mathcal{L}^{*}\left(\right.$ data $\left.\mid \beta_{H W W}\right)$ is the reduced likelihood and $\pi\left(\beta_{H W W}\right)$ is the prior p.d.f. for $\beta_{H W W}$. In this analysis we use a flat prior (in the form of a Heaviside function for the prior, i.e. $\pi\left(\beta_{H W W}\right)=H\left(\beta_{H W W}\right)$ with the Heaviside function $\left.\mathrm{H}\right)$.

To set an upper limit on the $H$ production cross-section, we integrate the posterior probability to cover $95 \%$ [18]. The expected and observed results are shown in Table 8 and in Figure 48 .

The upper limits at 95\% C.L. on the $H$ production cross section times the branching ratio are between 7.7 and 62.1 times the Standard Model, while the expected limits estimated in pseudo-experiments are between 12.5 and 41.0 times the Standard Model. 

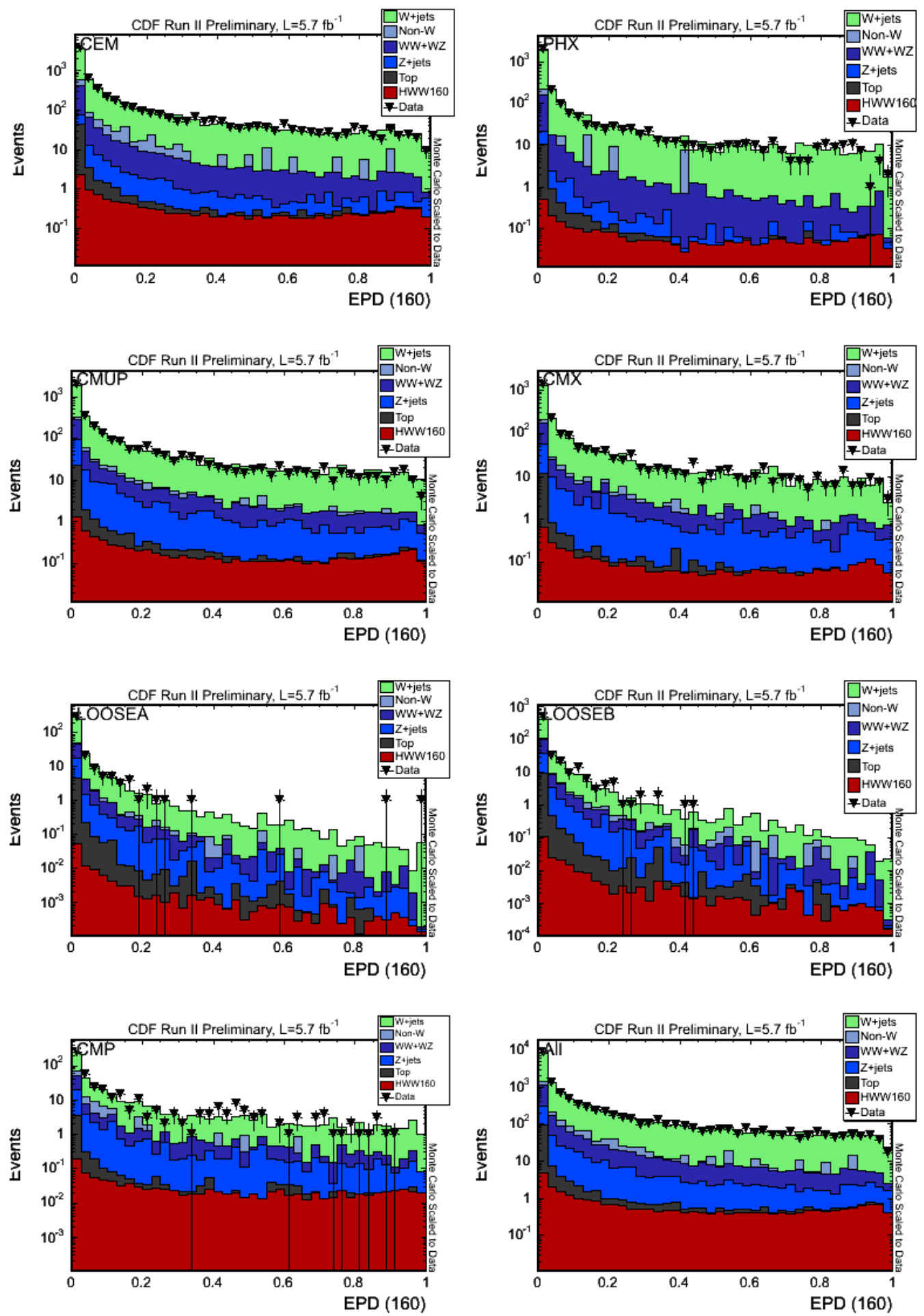

Figure 47: Comparison of the EPD output for data and the Monte Carlo prediction for $H \rightarrow W W$ (with $m_{H}=160 \mathrm{GeV}$ ) signal and background for the different categories. Note that the scale is adapted for the LOOSE categories and for CMP; PHX is shown for completeness only, it is not included in the analysis. 


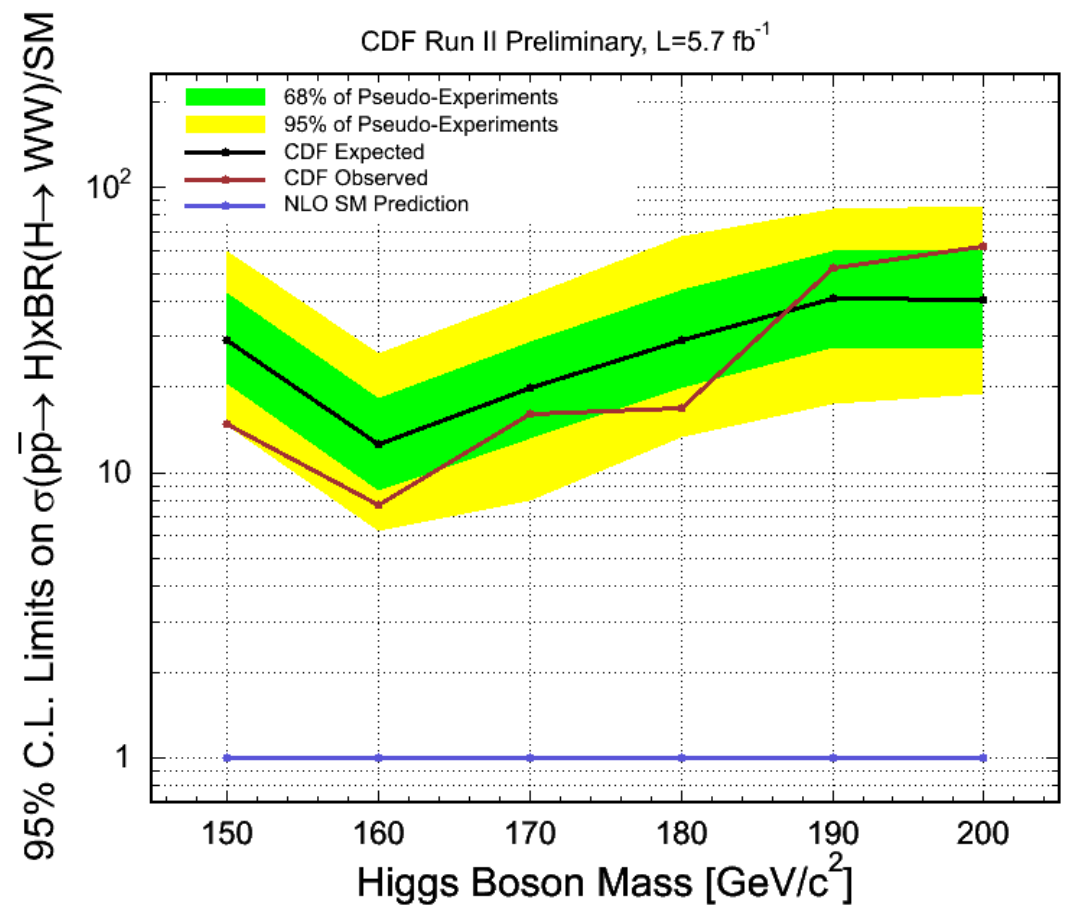

Figure 48: 95\% C.L. upper limits on the $H$ cross section multiplied by the $H \rightarrow W W$ branching ratio for Higgs boson masses of 150, 160, 170, 180, 190, and $200 \mathrm{GeV} / c^{2}$. The plot shows the limit normalized to the predictions from the Standard Model.

\begin{tabular}{|l|c|c|c|c|c|c|}
\hline$\sigma / \mathrm{SM}$ & $150 \mathrm{GeV}$ & $160 \mathrm{GeV}$ & $170 \mathrm{GeV}$ & $180 \mathrm{GeV}$ & $190 \mathrm{GeV}$ & $200 \mathrm{GeV}$ \\
\hline Expected & 29.2 & 12.5 & 19.9 & 29.3 & 41.0 & 40.2 \\
\hline Observed & 14.9 & 7.7 & 16.1 & 16.9 & 52.1 & 62.1 \\
\hline
\end{tabular}

Table 8: Expected and observed upper limit cross section, in SM units, for all Higgs mass points. 


\subsection{Effect of Systematic Uncertainties}

\subsubsection{Upper Limits without Systematic Uncertainties}

In order to evaluate the effect the systematic uncertainties previously described in Section 7 have on the expected limits, the following uncertainties were added one by one:

- Uncertainty on the $W+$ jets contribution normalization (" $W+$ jets")

- Uncertainty on the jet energy scale ("JES")

- Uncertainty due to factorization/renormalization scale (" $Q{ }^{2}$ ")

The other uncertainties listed in Table 7 remained included. The expected limits, without those three uncertainties, are shown in Table 9.

\begin{tabular}{|l|c|c|c|c|c|c|}
\hline$\sigma / \mathrm{SM}$ & $150 \mathrm{GeV}$ & $160 \mathrm{GeV}$ & $170 \mathrm{GeV}$ & $180 \mathrm{GeV}$ & $190 \mathrm{GeV}$ & $200 \mathrm{GeV}$ \\
\hline Expected & 19.8 & 8.6 & 11.1 & 15.7 & 22.8 & 23.9 \\
\hline
\end{tabular}

Table 9: Expected upper limits without uncertainties on $W+$ jets normalization, jet energy scale or on the factorization/renormalization scale

\subsubsection{Effect of Uncertainty on the $W+$ jets Normalization}

When including the uncertainty on the $W+$ jets normalization (while still excluding the uncertainty on the jet energy scale and the factorization/renormalization scale), one obtains the expected limits given in Table 10. On average, the expected limits increase by $14 \%$ due to the uncertainty on the $W+$ jets normalization.

\begin{tabular}{|l|c|c|c|c|c|c|}
\hline$\sigma / \mathrm{SM}$ & $150 \mathrm{GeV}$ & $160 \mathrm{GeV}$ & $170 \mathrm{GeV}$ & $180 \mathrm{GeV}$ & $190 \mathrm{GeV}$ & $200 \mathrm{GeV}$ \\
\hline Expected & 22.5 & 9.3 & 12.8 & 18.7 & 27.2 & 25.6 \\
\hline
\end{tabular}

Table 10: Expected upper limits including the uncertainty on the $W+$ jets normalization, but neither the uncertainty on the jet energy scale nor on the factorization/renormalization scale

\subsubsection{Effect of Uncertainty on Jet Energy Scale}

When not only the uncertainty on the $W+$ jets normalization is included but also the uncertainty on the jet energy scale ("JES"), one finds the expected limits shown in Table 11. A comparison of the expected limits listed in Table 11 with those in Table 10 reveals that the uncertainty on the jet energy scale translates to an increase in the expected limits by $13 \%$. The combined effect of the uncertainty on the $W+$ jets normalization and the jet energy scale is on the order of 29\%, averaged over all Higgs masses. 


\begin{tabular}{|l|c|c|c|c|c|c|}
\hline$\sigma / \mathrm{SM}$ & $150 \mathrm{GeV}$ & $160 \mathrm{GeV}$ & $170 \mathrm{GeV}$ & $180 \mathrm{GeV}$ & $190 \mathrm{GeV}$ & $200 \mathrm{GeV}$ \\
\hline Expected & 24.2 & 10.2 & 14.4 & 21.6 & 30.8 & 31.4 \\
\hline
\end{tabular}

Table 11: Expected upper limits only with uncertainty on $W+$ jets normalization and the jet energy scale, but not the uncertainties on the factorization/renormalization scale

\subsubsection{Effect of Uncertainty on Factorization/Renormalization Scale}

When including all systematic uncertainties, including the uncertainty on the factorization/renormalization scale, we find the expected limits previously given in Table 8 . One can thus conclude that the $Q^{2}$ uncertainty has an impact on the order of $30 \%$ on the limits (averaged over all Higgs masses). Since the effect is so large, the expected limits for one-sided uncertainties were calculated (i.e. only varying $Q^{2}$ up and in a second run only varying it down). Overall, the down variation increased the expected limits more than the up variation, which is true only to a limited degree at higher masses where the effect of the up variation also becomes sizeable. Furthermore, the ratio of the up-corrected to the central shape and the ratio of the down-corrected to the central shape were smoothed for the $W+$ jets templates and the limits were calculated; there was no significant difference. Additionally, fits were attempted to said ratios (linear and polynomial) which did not seem to describe the ratio very well. The unfitted and unsmoothed templates were used in the calculation of the limits (while using the internal median smoothing algorithm in the MCLIMIT package).

\subsubsection{Summary of Effects of Systematic Uncertainties}

When we compare the different results side-by-side as in Table 12 , adding uncertainties step-by-step (though it should be noted that the effect is dependent on the order in which the uncertainties are added), one can see clearly that the largest effect stems from the factorization/renormalization scale (about 30\%), whereas both the uncertainty on the jet energy scale and the uncertainty on the $W+$ jets normalization have effects between $13-14 \%$.

\begin{tabular}{|l|c|c|c|c|c|c|}
\hline$\sigma /$ SM & 150 & 160 & 170 & 180 & 190 & 200 \\
\hline No systematic uncertainties & 19.8 & 8.6 & 11.1 & 15.7 & 22.8 & 23.9 \\
\hline "W+jets" only & 22.5 & 9.3 & 12.8 & 18.7 & 27.2 & 25.6 \\
\hline " $W+$ jets" and "JES" & 24.2 & 10.2 & 14.4 & 21.6 & 30.8 & 31.4 \\
\hline " $W+$ jets", "JES" and $Q^{2}$ & 29.2 & 12.5 & 19.9 & 29.3 & 41.0 & 40.2 \\
\hline
\end{tabular}

Table 12: Summary of expected upper limit cross section, in SM units, for different Higgs masses (indicated in $\mathrm{GeV}$ ), with systematic uncertainties added step by step. 


\subsection{Effect of the Addition of the Phi Gap Trigger}

As previously mentioned in Section 3 , the inclusion of the phi gap trigger added several anticipated Higgs events; an average overall gain on the order of $2.6 \%$ compared to the sum of all lepton categories was found (when compared only to the relevant data periods, the average gain was $3.1 \%$ ). To find out what improvement in sensitivity the addition of the phi gap trigger corresponds to, the limits were calculated for all data periods with and without the CMP category. While the improvement for $m_{H}=170 \mathrm{GeV}$ was almost zero $(0.1 \%)$, it was sizeable for most other mass points (up to $7.3 \%$ for $m_{H}=190 \mathrm{GeV}$ ). On average, the sensitivity was improved by $3.3 \%$. The improvements in sensitivity for all mass points are found in Table 13 .

\begin{tabular}{|l|c|c|c|c|c|c|}
\hline$\sigma / \mathrm{SM}$ & 150 & 160 & 170 & 180 & 190 & 200 \\
\hline expected (incl. CMP) & 29.8 & 13.0 & 19.9 & 30.1 & 44.0 & 41.9 \\
\hline expected (excl. CMP) & 29.2 & 12.5 & 19.9 & 29.3 & 41.0 & 40.2 \\
\hline improvement & $2.1 \%$ & $3.5 \%$ & $0.1 \%$ & $2.6 \%$ & $7.3 \%$ & $4.2 \%$ \\
\hline
\end{tabular}

Table 13: Expected upper limit cross section, in SM units for different Higgs masses (indicated in $\mathrm{GeV}$ ) with and without the additional CMP category; The improvement in sensitivity is also shown in the third row (calculated using four significant digits) 


\section{Conclusions and Outlook}

In this Master thesis, a direct search for the Standard Model Higgs boson production is presented. The final state of this channel, an identified electron or muon and two jets, is given by the Higgs boson decaying to two $W$ of which one decays hadronically and one leptonically to either a muon or an electron (with the corresponding neutrino).

The $H \rightarrow W W$ channel is the most promising channel in the region of high mass Higgs searches, i.e. $m_{H}>135 \mathrm{GeV}$, at the Tevatron. Since this analysis does not rely on both $W$ decaying leptonically, the combined branching ratio is significantly larger, which however also entails much more dominant backgrounds. If a Higgs boson is produced via gluon fusion $(g g \rightarrow H)$, the QCD background is overwhelming, hence the requirement of a muon or electron from a $W$ in the final state.

The data used in this analysis has been collected between February 2002 and January 2009 by the CDF II detector and corresponds to $5.7 \mathrm{fb}^{-1}$ of data as can be seen in Fig. 12. Five different triggers of central and forward leptons have been used. Adding the phi gap trigger lead to an increase in expected Higgs events on the order of $2.6 \%$ overall (for all data periods), or about $10 \%$ of the CMUP, translating to an improvement of up to $7.3 \%$ in the sensitivity (depending on the Higgs mass) and on average $3.3 \%$.

The selection of events has been done by requiring a high $p_{T}$ lepton, large missing transverse energy $\left(E_{T}>20 \mathrm{GeV}\right)$, and two energetic central jets in the final state.

Several other processes have similar or the same signature as the signal process and thus also need to be considered. Background contributions come from electroweak processes, $t \bar{t}$, single top production, $W+$ jets, and non- $W$ processes. These processes have been estimated using a combination of Monte Carlo calculations and independent measurements in control data samples.

Since the number of signal events after the event selection is much smaller than the uncertainty in the background prediction, a matrix element technique has been used, in which event probability densities for the signal and background processes are calculated. In the event probability calculation, several aspects need to be taken into consideration, such as that the interactions are initiated by partons, the low cross section of the neutrino, and the energy resolution of the detector. A mapping, called the transfer function, between the parton energy $\left(E_{p}\right)$ and the jet energy $\left(E_{j}\right)$ is used. Once the event probabilities for all events in the analysis have been calculated, they are combined to create a powerful discriminator called the Event Probability Discriminant (EPD).

Unfortunately, no evidence for a Higgs boson signal has been observed in an integrated luminosity of $5.7 \mathrm{fb}^{-1}$ of CDF Run II data. 95\% confidence upper limits on the Higgs production cross section are set. To extract the most probable Higgs content in the data, the signal and background EPD distributions have been fitted to the CDF data using a binned likelihood function. The upper limits on the Higgs production cross section times the branching ratio in units of the Standard Model prediction, of the Higgs boson 


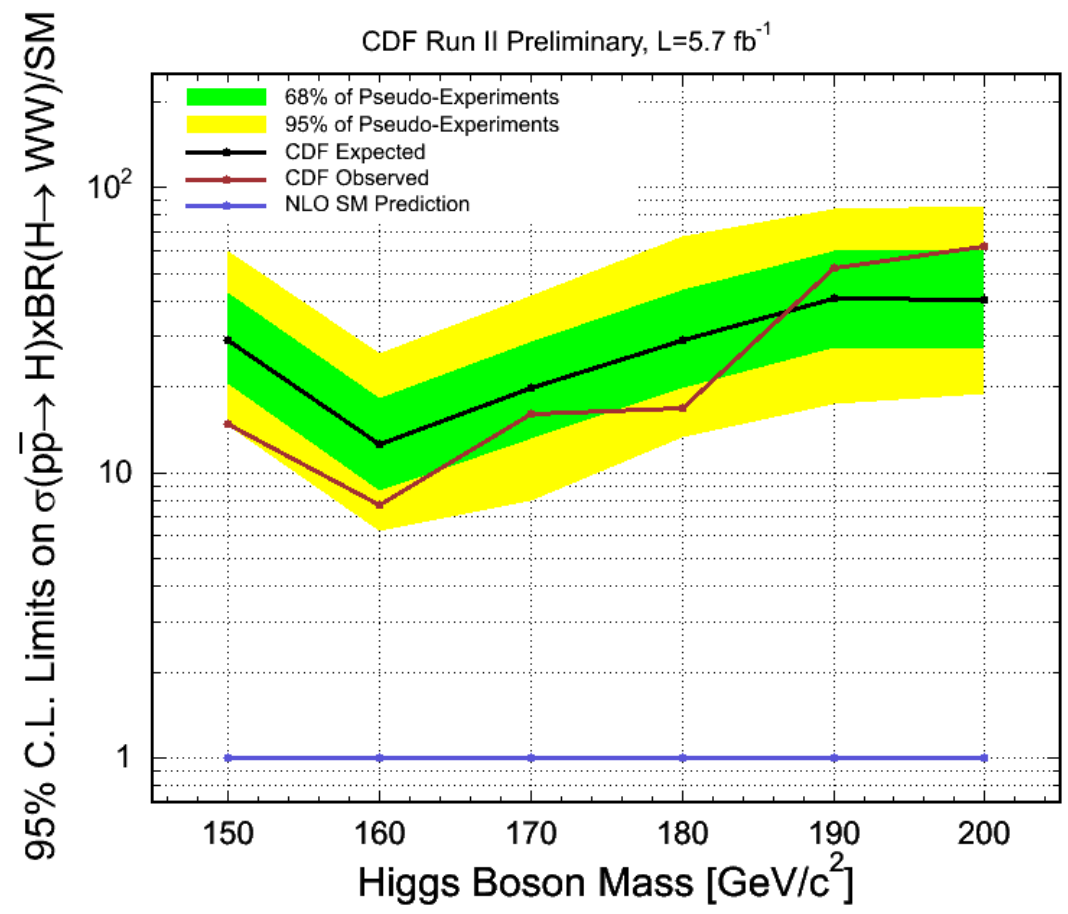

Figure 49: 95\% C.L. upper limits on the $H$ cross section multiplied by the $H \rightarrow W W$ branching ratio for Higgs boson masses of 150, 160, 170, 180, 190, and $200 \mathrm{GeV} / c^{2}$. The plot shows the limit normalized to the predictions from the Standard Model.

to decay to two $W$ bosons are given by $\sigma(p \bar{p} \rightarrow H) \times B R(H \rightarrow W W) / S M<7.7$ to 62.1 for Higgs boson masses between 150 and $200 \mathrm{GeV}$. The expected sensitivity estimated in pseudo-experiments is 12.5 to 41.0 at $95 \%$ confidence level. This result is summarized in Tab. 8 and in Fig. 49 .

The steps undertaken to include the phi gap trigger are documented separately so that in the future, additional triggers (such as the $\eta$ gap trigger) may be added in a similar fashion as the phi gap trigger to further improve sensitivity. 


\section{Acknowledgements}

I would like to thank Prof. Günther Dissertori and Prof. Rainer Wallny for making this thesis at Fermilab possible and also for the very generous financial support. I'm also deeply indebted to Prof. Craig Group for the countless hours he spent helping me and explaining triggers and various aspects of the detectors, and our inspiring 7:30 am status update conversations. Furthermore I'm very thankful for all the help provided by Dr. Martina Hurwitz who guided me through the analysis and introduced me to the tools necessary to carry it out. Thanks also go to the whole ME group for the very warm welcome, all the invaluable input and also for patiently enduring my sometimes endless weekly presentations. A special thank you goes to Florencia Canelli for all her support.

I'd also like to thank all the people who have enriched my stay with amusing and unforgettable aspects, be it a group meeting in Spanish, a trip to the beach or a $3 \mathrm{am}$ conversation about Quantum Electrodynamics with people from the dorm.

Last but not least I'd like to express my gratitude for all the support I received from my parents, my sister, my grandparents and my girlfriend. Their continued support was very important to me and I could not have done it without them. 


\section{A Higgs Field and Gauge Boson \& Fermion Masses}

Local symmetry implies the existence of massless vector particles, in stark contrast with experimental finding which have shown that the vector particles are in fact very heavy. The symmetries can however not be arbitrarily broken (as this would lead to the loss of renormalizability of the theory), e.g. simply introducing a mass-term is not allowed. It is important to note that, as shown by 't Hooft (1971b) gauge theories are renormalizable even in the presence of spontaneous symmetry breaking.

The Higgs boson is a Goldstone boson arising from the spontaneous breaking ${ }^{5}$ of the electroweak symmetry.

In the case of Abelian $U(1)$ gauge theory, one starts by considering a complex scalar field $\phi=\frac{1}{\sqrt{2}}\left(\phi_{1}+i \phi_{2}\right)$ (with the real fields $\phi_{1}$ and $\left.\phi_{2}\right)$ with a local $U(1)$ transformation

$$
\phi \rightarrow \phi^{\prime}=\phi e^{i e \alpha(x)}
$$

Gauge invariance demands the existence of the covariant derivative $D_{\mu}=\partial_{\mu}+i e A_{\mu}$, where $A_{\mu}$ is the massless $U(1)$ gauge field with

$$
A_{\mu} \rightarrow A_{\mu}^{\prime}=A_{\mu}-\partial_{\mu} \alpha(x)
$$

with the gauge invariant Lagrangian

$$
\mathcal{L}=\left(\partial^{\mu}-i e A^{\mu}\right) \phi^{*}\left(\partial_{\mu}+i e A_{\mu}\right) \phi \underbrace{-\mu^{2} \phi^{*} \phi-\lambda\left(\phi^{*} \phi\right)^{2}}_{-V(\phi)}-\frac{1}{4} F_{\mu \nu} F^{\mu \nu}
$$

and the field tensor

$$
F_{\mu \nu}=\partial_{\mu} A_{\nu}-\partial_{\nu} A_{\mu}
$$

The vacuum is a circle in the $\phi_{1}, \phi_{2}$ plane located at

$$
|\phi|^{2}=\left(\phi_{1}\right)^{2}+\left(\phi_{2}\right)^{2}=-\frac{\mu^{2}}{2 \lambda}=: v^{2}
$$

and we can expand $\phi$ about the vacuum as

$$
\phi(x)=\frac{1}{\sqrt{2}}(v+\eta(x)+i \xi(x))
$$

where $\eta(x)+i \xi(x)$ are the quantum fluctuations about the vacuum. This yields

$\mathcal{L}=\frac{1}{2}\left(\partial_{\mu} \xi\right)^{2}+\frac{1}{2}\left(\partial_{\mu} \eta\right)^{2}-v^{2} \lambda \eta^{2}+\frac{1}{2} e^{2} v^{2} A_{\mu} A^{\mu}+e v A_{\mu} \partial^{\mu} \xi-\frac{1}{4} F_{\mu \nu} F^{\mu \nu}+$ interaction terms

One then finds the following particle spectrum:

- massless scalar field $\xi$ (Goldstone boson)

\footnotetext{
${ }^{5}$ spontaneous breaking: the current is conserved, but the vacuum state is not invariant under the action of the corresponding charges
} 
- massive scalar field $\eta$ with mass $m_{\eta}=\sqrt{2 \lambda v^{2}}$

- massive $U(1)$ vector field $A_{\mu}$ with $m_{A}=e v$

There is a non-diagonal entry $\left(e v A_{\mu} \partial^{\mu} \xi\right)$ present in the expression ${ }^{6}$ The term will bring about a mixing of $A_{\mu}$ and $\xi$, making the interpretation less clear. One thus parametrizes the complex field in polar variables (shifting only the modulus field),

$$
\phi(x)=\frac{1}{\sqrt{2}}(v+h(x)) \exp \left(i \frac{\xi(x)}{v}\right)
$$

The Goldstone field $\xi$ from before is incorporated into $A_{\mu}$,

$$
A_{\mu} \rightarrow \hat{A}_{\mu}=A_{\mu}-\frac{1}{v} \partial_{\mu} \xi(x)
$$

which leads to the Lagrangian

$$
\mathcal{L}=\frac{1}{2}\left(\partial_{\mu} h\right)^{2}-\lambda v^{2} h^{2}+\frac{1}{2} e^{2} v^{2} \hat{A}_{\mu}^{2}-\lambda v h^{3}-\frac{1}{4} \lambda h^{4}+\frac{1}{2} e^{2} \hat{A}_{\mu}^{2} h^{2}+v e^{2} \hat{A}_{\mu}^{2} h-\frac{1}{4} \hat{F}_{\mu \nu} \hat{F}^{\mu \nu}
$$

The gauge has thus been fixed (the unitary gauge) and one finds the following particle spectrum

- massive scalar field $h$ with $m_{h}=\sqrt{2 \lambda v^{2}}$ (unconstrained)

- Goldstone field absorbed in $\hat{A}_{\mu}$ (giving longitudinal degree of freedom to $\hat{A}_{\mu}$ !)

- massive $U(1)$ vector field $\hat{A}_{\mu}$ with $m_{\hat{A}}=e v$

The number of degrees of freedom remains the same (originally, $\phi$ and $A_{\mu}$ each had 2 degrees of freedom, while after spontaneous symmetry breaking one has only one scalar field and one massive gauge boson, yet the latter has three polarization states yielding again a total of four degrees of freedom).

\section{A.1 Gauge Boson Masses in $S U(2) \otimes U(1)$}

To generate masses for the gauge bosons, one starts with the covariant derivative in $S U(2) \otimes U(1)$

$$
D_{\mu}=\partial_{\mu}-i g \frac{1}{2} \vec{\tau} \vec{W}_{\mu}-i g^{\prime} \frac{1}{2} Y B_{\mu}
$$

with $\overrightarrow{W^{\mu}}=\left(W_{1}^{\mu}, W_{2}^{\mu}, W_{3}^{\mu}\right)\left(W_{3}^{\mu}\right.$ and $B^{\mu}$ are not mass eigenstates but give us a mass eigenstate via the Weinberg angle). The Lagrangian of the complex scalar field is

$$
\mathcal{L}=\left[-i D^{\mu} \phi^{\prime}\right]^{\dagger}\left[i D_{\mu} \phi\right]-\mu^{2} \phi^{\dagger} \phi-\lambda\left[\phi^{\dagger} \phi\right]^{2}
$$

with $\phi$ being a $S U(2)$ doublet. Choose $Y=1$, write

$$
\phi=\frac{1}{\sqrt{2}}\left(\begin{array}{c}
\phi_{1}+i \phi_{2} \\
\phi_{3}+i \phi_{4}
\end{array}\right)=\left(\begin{array}{c}
\phi^{+} \\
\phi^{0}
\end{array}\right)
$$

${ }^{6}$ This is due to the choice of $\phi(x)=\frac{1}{\sqrt{2}}(v+\eta(x)+i \xi(x))$ which implicitly fixes the gauge of $\phi$ 
where we have again $\mu^{2}<0, \lambda>0, v^{2}=-\frac{\mu^{2}}{2 \lambda}$ and $\phi=\frac{1}{\sqrt{2}}\left(\begin{array}{c}0 \\ v+h(x)\end{array}\right)$ which is electrically neutral. We thus see that the vacuum breaks the $S U(2)_{L}$ and $U(1)_{Y}$ symmetries while $U(1)_{Q}$ is conserved since

$$
Q \phi=\left(T_{3}+\frac{1}{2} Y\right) \phi=0 \quad \text { and } \quad \phi^{\prime}=e^{i Q \alpha(x)} \phi=e^{i 0} \phi
$$

We find the particle spectrum from $\left[D_{\mu} \phi\right]^{\dagger}\left[D^{\mu} \phi\right]$ for $\phi=\frac{1}{\sqrt{2}}\left(\begin{array}{l}0 \\ v\end{array}\right)$ :

$$
\begin{aligned}
\left|\left(-i g \frac{\vec{\tau}}{2} \vec{W}_{\mu}-i g^{\prime} \frac{1}{2} Y B_{\mu}\right) \phi\right|^{2} & =\frac{1}{8}\left|\left(\begin{array}{cc}
g W_{\mu}^{3}+g^{\prime} B_{\mu} & g\left(W_{\mu}^{1}-i W_{\mu}^{2}\right) \\
g\left(W_{\mu}^{1}+i W_{\mu}^{2}\right) & -g W_{\mu}^{3}+g^{\prime} B_{\mu}
\end{array}\right)\left(\begin{array}{c}
0 \\
v
\end{array}\right)\right|^{2} \\
& =\frac{1}{8} v^{2} g^{2}\left|\left(W_{\mu}^{1}\right)^{2}+\left(W_{\mu}^{2}\right)^{2}\right|+\frac{1}{8} v^{2}\left(g^{\prime} B_{\mu}-g W_{\mu}^{3}\right)\left(g^{\prime} B^{\mu}-g W^{2 \mu}\right) \\
& =\left(\frac{1}{2} v g\right)^{2} W_{\mu}^{+} W^{\mu-}+\frac{1}{8} v^{2}\left(g^{\prime} B_{\mu}-g W_{\mu}^{3}\right)^{2} \\
& =m_{W}^{2} W_{\mu}^{+} W^{\mu-}+\frac{1}{2} m_{Z}^{2} Z_{\mu} Z^{\mu}
\end{aligned}
$$

so $m_{W}=\frac{1}{2} v g$ and $m_{Z}=\frac{1}{2} v \sqrt{g^{2}+g^{\prime 2}}\left(\right.$ where $Z^{\mu}=\left(g W_{3}^{\mu}-g^{\prime} B^{\mu}\right) / \sqrt{g^{2}+g^{\prime 2}}$ has been used).

\section{A.2 Fermion Masses}

As an example of fermion masses, one can consider the electron $\left(e_{L}\right.$ has $\left.T_{3}=-\frac{1}{2}\right)$. Fermion masses are to be obtained in a gauge-invariant fashion (through the Higgs mechanism). The same Higgs doublet needs to generate fermion and gauge boson masses.

One starts by including the $(S U(2) \otimes U(1))$ gauge invariant term

$$
\left.\mathcal{L}_{m}=-G_{e}\left[\begin{array}{ll}
\left(\bar{\nu}_{e}\right. & \bar{e}
\end{array}\right)_{L}\left(\begin{array}{c}
\phi^{+} \\
\phi^{0}
\end{array}\right) e_{R}+\bar{e}_{R}\left(\bar{\phi}^{+} \bar{\phi}^{0}\right)\left(\begin{array}{c}
\nu_{e} \\
e
\end{array}\right)_{L}\right] \quad, \quad \text { with }\left(\begin{array}{c}
\phi^{+} \\
\phi^{0}
\end{array}\right)=\frac{1}{\sqrt{2}}\left(\begin{array}{c}
0 \\
v+h(x)
\end{array}\right)
$$

where $G_{e}$ is the electron Yukawa coupling. We thus have

$$
\mathcal{L}_{m}=-\frac{G_{e}}{\sqrt{2}} v\left(\bar{e}_{L} e_{R}+\bar{e}_{R} e_{L}\right)-\frac{G_{e}}{\sqrt{2}} h\left(\bar{e}_{L} e_{R}+\bar{e}_{R} e_{L}\right)=-m_{e} \bar{e} e-\frac{m_{e}}{v} \bar{e} e h
$$

where $m_{e}=\frac{1}{\sqrt{2}} G_{e} v$. While the masses of the fermions are not predicted (they are free parameters of the Standard Model), one can see from (23) that the coupling of the electron to the Higgs is proportionate to its mass. The same procedure is also valid for $T_{3}=-\frac{1}{2}$ fermions.

To generate mass for the upper member of a quark doublet $\left(T_{3}=+\frac{1}{2}\right)$ one uses

$$
\phi^{c}=-i \tau_{2} \phi^{*}=\left(\begin{array}{c}
-\bar{\phi}^{0} \\
\bar{\phi}^{+}
\end{array}\right)=\frac{1}{\sqrt{2}}\left(\begin{array}{c}
v+h(x) \\
0
\end{array}\right)
$$


with $Y=-1$ which yields (one family formulation)

$$
\begin{aligned}
& \left.\mathcal{L}=-G_{d}\left[\begin{array}{ll}
\bar{u} & \bar{d}
\end{array}\right)_{L}\left(\begin{array}{c}
\phi^{+} \\
\phi^{0}
\end{array}\right) d_{R}+\bar{d}_{R}\left(\bar{\phi}^{+} \quad \bar{\phi}^{0}\right)\left(\begin{array}{l}
u \\
d
\end{array}\right)_{L}\right] \\
& \left.-G_{u}\left[\begin{array}{ll}
\bar{u} & \bar{d}
\end{array}\right)_{L}\left(\begin{array}{c}
-\phi^{0} \\
\phi^{+}
\end{array}\right) u_{R}+\bar{u}_{R}\left(\begin{array}{ll}
-\bar{\phi}^{0} & \bar{\phi}^{+}
\end{array}\right)\left(\begin{array}{l}
u \\
d
\end{array}\right)_{L}\right] \\
& =-m_{d} \bar{d} d-\frac{m_{d}}{v} \bar{d} d h-m_{u} \bar{u} u-\frac{m_{u}}{v} \bar{u} u h
\end{aligned}
$$

where we see again that the coupling is proportionate to the mass of the particle. 


\section{References}

[1] D. Benjamin et al., Updated search for $\mathrm{H}$ to WW Production Using $3.6 \mathrm{fb}^{-1}$, CDF/PHYS/EXOTIC/CDFR/9697 (2009).

[2] DØCollaboration, Operation and Physics Potential of Tevatron Run 2, EPJ direct, 4(0):1-10 (2002).

[3] Fermilab Beams Division, Accelerator Details, http://www-bd.fnal.gov/ public/tevatron.html (2000).

[4] The DØCollaboration, Run II luminosity, http://www-d0.fnal.gov/runcoor/ RUN/run2_lumi.html (2006).

[5] Taka Yasuda, Electroweak Physics at Tevatron, Talk given at 5th European Meeting from the Planck Scale to the Electroweak Scale (2002).

[6] The CDF II Collaboration, The CDF II Detector Technical Design Report, FERMILAB-Pub-96/390-E (2001).

[7] CDF Collaboration, CDF Run 2 Silicon Detector, 2006 IEEE Nuclear Science Symposium Conference Record (2006).

[8] CDF Collaboration, Calorimetry in CDF Run 2, Proceedings of ICHEP 2002, pp.903-905 (2002).

[9] CDF Collaboration, CDF Run 2 muon system, Eur Phys J C 33, s01, s1002s1004 (2004).

[10] John Conway, Tevatron Run 2 Cross Sections and Branching Ratios, http: //www.physics.ucdavis.edu/〜conway/research/higgs/smhiggs-tev.html (2003).

[11] A.Djouadi, J.Kalinowski and M.Spira, Comp. Phys. Commun. 108 C (1998) 56, arXiv: hep-ph/9704448v1 (1997).

[12] P Teixeira-Dias, Higgs boson searches at LEP, J. Phys.: Conf. Ser. 110042030 (2008).

[13] J. Pumplin et al., New generation of parton distributions with uncertainties from global QCD analysis, JHEP, 0207:012 (2002)

[14] L. Collaborations, Precision Electroweak Measurements and Constraints on the Standard Model, arXiv:0911.2604 [hep-ex] (2009).

[15] The CDF-II Collaboration, A Time-of-Flight Detector in CDF II, doi:10.1016/j.nima.2003.11.097 (2003)

[16] T.LeCompte, H.T. Diehl, The CDF and D $\varnothing$ Upgrades for Run II, Annu. Rev. Nucl. Part. Sci. 2000.50:71-117 (2000). 
[17] M. Hurwitz, Measurement of the $\mathrm{WW}+\mathrm{WZ}$ production cross section in a semileptonic decay mode at CDF, CDF/THESIS/EXOTIC/PUBLIC/10124 (2010).

[18] Particle Data Group, The Review of Particle Physics, http://pdg.1bl.gov (2006).

[19] F. Canelli, B. Casal Larana, C. Group, A. Ruiz, B. Stelzer, R. Vilar, Increasing Muon Acceptance with MET Plus Jet Triggers, CDF Note 9105 (2008).

[20] S.Rolli and L.Lovas, Muon id, reconstruction and trigger efficiencies and scale factors for period 9-12 data, CDF/ANAL/MUON/CDFR/9085 (2007).

[21] Martina Hurwitz, Florencia Canelli, Ricardo Eusebi, Craig Group, Enrique Palencia, Bruno Casal, Barbara Alvarez, Bernd Stelzer, Measurement of WW+WZ production cross section in l $\nu \mathrm{jj}$ channel using $4.6 \mathrm{fb}^{-1}$, CDF Note 9923 (2009).

[22] Florencia Canelli, Bruno casal, Craig Group, Alberto Ruiz, Bernd Stelzer, Rocio Vilar, Increasing Muon Acceptance with the MET Plus Jet Triggers, CDF Note 9106 (2007).

[23] Tom Junk et al., Combined CDF and DZero Upper Limits on Standard Model Higgs Boson Production with up to $6.7 \mathrm{fb}^{-1}$ of data, CDF/PHYS/EXOTIC/PUBLIC/10241 (2010).

[24] D. Acosta et al., First Measurements of Inclusive W and Z Cross Sections from Run II of the Fermilab Tevatron Collider, Phys. Rev. Lett. 94, 091803 (2005).

[25] L. Demortier, Bayesian treatments of Systematic Uncertainties, Proceedings of Advanced Statistical Techniques in Particle Physics, Grey College, Durham, 18 - 22 March 2002, http://www.ippp.dur.ac.uk/Workshops/02/statistics/proceedings.shtml.

[26] J. Adelman et al., Method II For You, CDF Note 9185 (2008).

[27] Sarah Budd, Matthias Buehler, Catalin Ciobanu, Peter Dong, Thomas Junk, Jan Lueck, Thomas Muller, Svenja Richter, Bernd Stelzer, Wolfgang Wagner, Rainer Wallny, Estimation and Modeling of Non-W Background for Single-Top Searches, CDF Note 8489 (2006).

[28] C. Amsler et al. (Particle Data Group), 2008 Review of Particle Physics, Phys. Lett., B667:1 (2008).

[29] T. Junk, Confidence level computation for combining searches with small statistics, Nucl. Instrum. Methods, A434:435 (1999)

[30] A.L. Read, Presentation of search results: the $\mathrm{CL}_{s}$ technique. J.Phys, G28:2693 (2002).

[31] CDF Collaboration, Combined Upper Limit on Standard Model Higgs Boson Production for Winter 2009, CDF Public note 9674 (2009). 